\title{
Network formation games
}

Citation for published version (APA):

Tennekes, M. (2010). Network formation games. [Doctoral Thesis, Maastricht University]. Maastricht University. https://doi.org/10.26481/dis.20100326mt

Document status and date:

Published: 01/01/2010

DOI:

10.26481/dis.20100326mt

Document Version:

Publisher's PDF, also known as Version of record

\section{Please check the document version of this publication:}

- A submitted manuscript is the version of the article upon submission and before peer-review. There can be important differences between the submitted version and the official published version of record.

People interested in the research are advised to contact the author for the final version of the publication, or visit the DOI to the publisher's website.

- The final author version and the galley proof are versions of the publication after peer review.

- The final published version features the final layout of the paper including the volume, issue and page numbers.

Link to publication

\footnotetext{
General rights rights.

- You may freely distribute the URL identifying the publication in the public portal. please follow below link for the End User Agreement:

www.umlib.nl/taverne-license

Take down policy

If you believe that this document breaches copyright please contact us at:

repository@maastrichtuniversity.nl

providing details and we will investigate your claim.
}

Copyright and moral rights for the publications made accessible in the public portal are retained by the authors and/or other copyright owners and it is a condition of accessing publications that users recognise and abide by the legal requirements associated with these

- Users may download and print one copy of any publication from the public portal for the purpose of private study or research.

- You may not further distribute the material or use it for any profit-making activity or commercial gain

If the publication is distributed under the terms of Article $25 \mathrm{fa}$ of the Dutch Copyright Act, indicated by the "Taverne" license above, 
Network Formation Games 
Network Formation Games

Copyright (c) 2009 Martijn Tennekes

Printed by Gildeprint, Enschede

ISBN 978-94-6108-008-0 


\title{
Network Formation Games
}

\author{
Proefschriwft
}

ter verkrijging van de graad van doctor aan de Universiteit Maastricht, op gezag van de Rector Magnificus, Prof. mr. G.P.M.F. Mols, volgens het besluit van het College van Decanen, in het openbaar te verdedigen op vrijdag 26 maart 2010 om 14.00 uur

door

Martijn Tennekes 


\section{Promotor:}

Prof. dr. ir. R. L. M. Peeters

\section{Copromotores:}

Dr. J. J. M. Derks

Dr. ir. J. Kuipers

Dr. F. Thuijsman

\section{Beoordelingscommissie:}

Prof. dr. H. J. M. Peters (voorzitter)

Dr. J. J. A. Kamphorst (Erasmus Universiteit Rotterdam)

Prof. dr. ir. G. van der Laan (Vrije Universiteit Amsterdam)

Prof. dr. A. M. Riedl 
People usually consider walking on water or in thin air a miracle. But I think the real miracle is not to walk either on water or in thin air, but to walk on earth. Every day we are engaged in a miracle which we don't even recognize: a blue sky, white clouds, green leaves, the black, curious eyes of a child - our own two eyes. All is a miracle.

- Thich Nhat Hanh - 



\section{Contents}

1 Introduction 1

1.1 Networks . . . . . . . . . . . . . . . . . . . . . . . . 1

1.2 Formation of networks $\ldots \ldots \ldots \ldots \ldots$

1.3 Outline of this thesis . . . . . . . . . . . . . . . . . 7

2 Nash networks in the one-way flow model 99

2.1 Model and notations . . . . . . . . . . . . . . . . . . . . . . . . 11

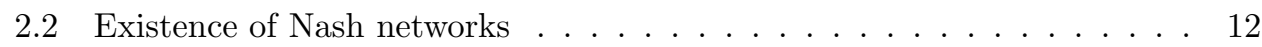

$2.2 .1 \quad$ Proof by induction . . . . . . . . . . . . . . . . . . . . 13

2.2 .2 Proof by construction $\ldots \ldots \ldots \ldots \ldots$

2.2 .3 Heterogeneous link costs $\ldots \ldots \ldots \ldots \ldots$

2.3 Architecture of Nash networks . . . . . . . . . . . . . . . . . . 24

2.4 Strict Nash networks . . . . . . . . . . . . . . . . . . . . . . . . . . . . . 27

$2.5 \quad$ Negative profits $\ldots \ldots \ldots \ldots \ldots \ldots$

2.6 Overview of the results . . . . . . . . . . . . . . . . . . . . . . . . . 34

3 Best response problem 35

3.1 Problem formulation . . . . . . . . . . . . . . . . . . . . 36

3.2 Complexity class $\ldots \ldots \ldots \ldots \ldots \ldots$

$3.3 \quad$ Algorithm ARRAC $\ldots \ldots \ldots \ldots$

3.4 Time and space complexity of ARRAC $\ldots \ldots \ldots \ldots$. . . . . . 44

3.5 Average case analysis of ARRAC $\ldots \ldots \ldots \ldots \ldots$

\begin{tabular}{llll}
\hline 3.6 & Two-way flow case & $\ldots$ & $\ldots$
\end{tabular}$\ldots \ldots \ldots$

4 Local- and global-Nash networks 51

4.1 Model and notations . . . . . . . . . . . . . . . . . 52

4.2 Local-Nash and global-Nash networks . . . . . . . . . . . . . . 54

4.3 Existence of local-Nash networks $\ldots \ldots \ldots \ldots \ldots$

4.4 Architecture of (strict) local-Nash networks . . . . . . . . . . . . 62

$4.5 \quad$ Property independence $\ldots \ldots \ldots \ldots \ldots$

4.6 Payoff functions satisfying the properties $\ldots \ldots \ldots$. . . . . . . 67 
4.7 Concluding remarks $\ldots \ldots \ldots \ldots \ldots$

\begin{tabular}{|lll}
5 & Dynamic network formation game & $\mathbf{7 1}$
\end{tabular}

5.1 Game description . . . . . . . . . . . . . . . . . . 72

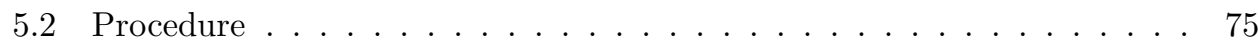

5.3 Concluding remarks $\ldots \ldots \ldots \ldots \ldots$

\begin{tabular}{|lll}
6 & Payoff properties in the one-way flow model & 81
\end{tabular}

6.1 Notations . . . . . . . . . . . . . . . . . . . . 82

6.2 Characterization . . . . . . . . . . . . . . . . . . . . 82

6.3 Downstream efficiency $\ldots \ldots \ldots \ldots \ldots$

$\begin{array}{lll}7 & \text { An axiomatic approach covering the two-way flow model } & 91\end{array}$

7.1 Model and notations $\ldots \ldots \ldots \ldots \ldots$

7.2 Axiomatization . . . . . . . . . . . . . . . . . . . . 94

7.3 Property independence $\ldots \ldots \ldots$. . . . . . . . . . . . . . . . . . . . . 99

7.4 Characterization . . . . . . . . . . . . . . . . . . . . . 101

7.5 Concluding remarks $\ldots \ldots \ldots$. . . . . . . . . . . . . 104

8 Project Support Games 105

8.1 Model . . . . . . . . . . . . . . . . . . . . . . . 106

8.2 Stability . . . . . . . . . . . . . . . . . . . . . . . 107

8.3 Examples . . . . . . . . . . . . . . . . . . . . . . . . . . 110

8.4 Nash equilibria . . . . . . . . . . . . . . . . . . . . . . . . . . . . . 112

8.5 Discussion . . . . . . . . . . . . . . . . . . . . . . . . . . . 113

$\begin{array}{llr}9 \text { Conclusion } & 115\end{array}$

9.1 Unilateral connections model . . . . . . . . . . . . . . . . . 115

9.2 Further research $\ldots \ldots \ldots \ldots \ldots$. . . . . . . . . . . . 118

\begin{tabular}{ll}
\hline Bibliography & 119
\end{tabular}

\begin{tabular}{ll}
\hline Notations and definitions & 125
\end{tabular}

$\begin{array}{ll}\text { Index } & 127\end{array}$

\begin{tabular}{ll}
\hline Samenvatting & 129
\end{tabular}

\begin{tabular}{ll}
\hline Acknowledgements & 133
\end{tabular}

\begin{tabular}{ll}
\hline About the author & 135
\end{tabular} 


\section{Chapter 1}

\section{Introduction}

This thesis deals with mathematical models of network formation. In this chapter, we provide an introduction and an overview of this thesis.

\section{$1.1 \quad$ Networks}

People face many different networks in everyday life, for instance transport, telecommunication, navigation, friendship, and computer networks. A network is a structure of nodes that are interconnected by links. For example, a node in a friendship network represents a person, and a link between two persons represents a friendship between them. As such, a network can be represented and depicted as a graph (see Figure 1.1).

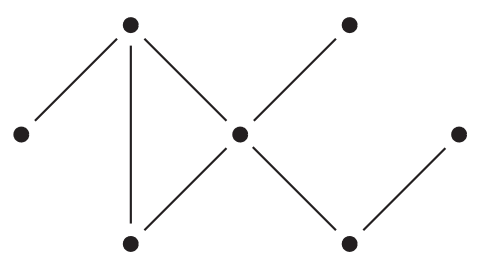

Figure 1.1: A network depicted as a graph. 
In Table 1.1. several examples of networks are listed with the interpretation of the corresponding nodes and links. Here, we distinguish two types of networks: endogenous and exogenous networks. This distinction is characterized by the way the networks are built.

\begin{tabular}{|c|c|c|}
\hline Network & Node & Link \\
\hline \multicolumn{3}{|l|}{ Endogenous networks } \\
\hline Friendship network & Person & Friendship \\
\hline Online social network ${ }^{1}$ & User profile & Inclusion in each other's contact list \\
\hline Scientific collaboration network & Scientist & Collaboration \\
\hline Trade network & Trader & Bilateral trade \\
\hline R\&D network & Firm & Joint R\&D activity \\
\hline World Wide Web & Website & Hyperlink (URL) \\
\hline \multicolumn{3}{|l|}{ Exogenous networks } \\
\hline Railway network & Railway station & Railway \\
\hline Electricity network & Power station or building & Power line \\
\hline Computer network (physical) & Computer, router, or server & Cable or wireless connection \\
\hline
\end{tabular}

Table 1.1: Examples of networks.

Endogenous networks are built by autonomous decision makers, who correspond to the nodes. For instance, the bilateral trades among a group of traders define a trade network. Although there may be trading rules (given by some external party), the traders themselves determine with whom they trade.

Exogenous networks are built by external parties. Generally speaking, the nodes in these networks represent objects rather than individuals. As an example, consider a network in which railway stations are interconnected by railways. A railway company designs and maintains this network.

As can be seen from Table 1.1. social and economic networks are typically endogenous, while transport and telecommunication networks are typically exogenous. Observe that a computer network can be either exogenous or endogenous, depending on its type. Physical computer networks and the Internet are exogenous, while the World Wide Web (WWW), a network of websites, is endogenous. Notice that the WWW uses the Internet as a medium.

\subsection{Formation of networks}

We are particularly interested in how endogenous networks are formed, and how their architectures are obtained. While exogenous networks can be formed by using optimization algorithms, endogenous networks are formed by the interaction of the individual nodes. To examine the formation of endogenous networks, we use simplified models

\footnotetext{
${ }^{1}$ Examples: MySpace, Facebook, Hyves, and LinkedIn (which is business oriented).
} 
from game theory, which attracted broad attention since Von Neumann and Morgenstern (1944) published their book 'Theory of Games and Economic Behavior'. These models provide insights in the basic principles of network formation, with the ultimate objective to study complex models that provide a better understanding of network formation in reality.

A game is an interactive decision problem of a group of rational agents (often referred to as players). The decisions of the agents during the game jointly determine the outcome of a play. Each possible outcome generates a payoff for each agent. A game is called a non-cooperative game if the agents are not able to make binding agreements. Otherwise, it is called a cooperative game.

We study network formation games, by which we mean non-cooperative games that are used to model the process of network formation. The agents who play such a network formation game correspond to the nodes of a network to be formed. Following the specific description of the game, they are able to link with other agents and thus form a network together.

A non-cooperative game is described by four elements. First, it is played by a set of agents. Second, there are specific rules that define how the game is played. These rules tell the agents what actions they may take. Third, there are several possible outcomes of the game. The outcome is determined by the actions that agents took during the game. Fourth, a payoff function assigns a value to each agent for each possible outcome. In Table 1.2 these four elements are applied to network formation games.

\begin{tabular}{ll}
\hline Elements of a non-cooperative game & Elements applied to a network formation game \\
\hline Agents & Agents who correspond to nodes \\
Rules & Rules that define how agents may form links \\
Outcomes & Formed networks ${ }^{1}$ \\
Payoff function & Function that assigns a payoff to each agent \\
& given the formed network \\
\hline
\end{tabular}

Table 1.2: Network formation games.

In the literature on network formation games, one focus is on equilibrium networks, i.e. networks where no agent can gain by a unilateral deviation. Do they exist? If so, under what conditions? What architectures do they have? In case dynamic network formation procedures are examined, central questions are the following. Do these procedures converge to equilibrium networks? If so, what is the speed of convergence? Moreover, what kind of networks are reached?

In this thesis we only study models with myopic agents, i.e. in choosing their actions, agents do not look far ahead. For a survey of network formation models with farsighted agents, we refer to Page and Kamat 2005).

\footnotetext{
1 Bloch and Jackson (2007) propose a model of network formation where the investments of the agents are part of the outcome and part of the payoff function. We discuss this model on page 6
} 


\section{Models using cooperative game theory}

A first approach to model network formation as a non-cooperative game is taken by Aumann and Myerson (1988). They define a game where the possible links are proposed to the agents one after the other. When a link is proposed, the two adjacent agents have the opportunity to form it. Once a link is formed, it cannot be removed afterwards. After all links have been proposed, all non-formed links are again proposed in the same order as in the first round. The adjacent agents get a final opportunity to form them. During the game, the history is known to all agents.

Myerson (1991) models network formation as a static non-cooperative game. In this game, the agents simultaneously announce to which other agents they want to form a link. A link is only formed if both agents have chosen it.

The payoffs in both games are determined by underlying cooperative games, in which the coalitions correspond to the components of the networks. An allocation rule assigns a payoff for each agent given the coalitional structure, i.e. the components of a network. Myerson (1977) introduces a frequently used allocation rule for these cooperative games. For an overview of this line of research, we refer to Van den Nouweland (2005).

\section{The bilateral connections model}

Jackson and Wolinsky (1996) introduce the bilateral connections model where the payoff function is derived directly from the architecture of the formed network. Each agent pays a certain cost for each adjacent link. Further, each agent receives a certain profit for each other agent to whom he is connected by a path between them in the network. The value of this profit decreases with the distance of the shortest path between them (measured in number of links).

As in the static network formation game proposed by Myerson (1991), links are formed bilaterally, i.e. when both adjacent agents agree. Jackson and Wolinsky (1996) introduce pairwise stability as an equilibrium concept. A network is called pairwise stable if no agent prefers to delete a link and if no pair of non-linked agents prefers to form a link between them.

Jackson and Wolinsky (1996) focus on the comparison between pairwise stable networks and efficient networks (networks for which the sum of payoffs over all agents is maximal). Several other equilibrium concepts are studied in the literature. For instance, Dutta and Muttuswami (1997) and Jackson and Van den Nouweland (2005) consider equilibrium concepts that also allow for coalitions larger than pairs to deviate. For a survey of this line of research, we refer to Jackson (2005).

\section{The unilateral connections model}

Bala and Goyal (2000a) introduce a unilateral variant of the connections model, where agents are able to form links without consent of other agents. Links are directed in 
this model. Each agent forms a set of own links, i.e. links pointing at him. The links of all agents together define the formed network.

Each agent pays costs for the links that he formed, and receives profits from other agents via the network. These profits are nonrival, i.e. if they are sent from agent $i$ to agent $j$, then they are still available to agent $i$. A good example of nonrival profits is information. Bala and Goyal (2000a) study two variants, namely the one-way flow model and the two-way flow model. In the one-way flow model, agent $i$ observes agent $j$, and therefore receives profits, if a directed path exists from $j$ to $i$, while in the two-way flow model, agent $i$ observes agent $j$ if an undirected path exists between them.

A network is called a Nash network if no agent can receive a higher payoff by unilaterally deviating from his set of links 1

Bala and Goyal (2000a) study a class of payoff functions that strictly decrease in the number of own links in the formed network, and strictly increase in the number of observed agents. They also consider a linear case, where each agent pays a fixed cost $c$ for each own link in the formed network and receives a profit normalized to 1 for each observed agent. They prove the existence of Nash networks for both the one- and the two-way flow model, and characterize them for the one-way flow model as being either directed cycles or empty (i.e. without links), and for the two-way flow model as being connected networks without (un)directed cycles.

Heterogeneity among link costs and profits is studied by Galeotti (2006) for the oneway flow model, and by Galeotti et al. (2006) and Haller et al. (2007) for the two-way flow model. The focus in these papers is on the architecture of strict Nash networks, i.e. networks in which any deviation gives a strictly lower payoff to the deviating agent. The existence of Nash networks for the two-way flow model with heterogeneous profits is proved by Haller et al. (2007).

The model is further extended by incorporating information decay, i.e. the value of profits that $i$ receives from observing $j$ decreases with the length of the shortest (un)directed path between them. Due to information decay, agents prefer to be connected to other agents via short paths. This extension is studied by Bala and Goyal (2000a), Kannan et al. (2007), and Feri (2007).

Bala and Goyal (2000b) extend the model in another way by assigning a reliability factor to each link. Each formed link actually functions with a certain probability. The agents still pay the costs for all formed links, but the value of profits depends on the reliability of the links. Therefore, agents prefer to be connected to other agents via several paths. They also prefer short paths. This extension is also studied by Haller and Sarangi (2005) and Haller et al. (2007).

A dynamic game based on this unilateral connections model is studied by Bala and Goyal (2000a). The initial network is chosen randomly among all possible networks. At the beginning of each time stage, a random set of agents is identified. Each of these agents has the opportunity to change his set of links. Then, the network is updated

\footnotetext{
${ }^{1}$ The concept of Nash equilibria for non-cooperative games is introduced by Nash 1950$)$.
} 
and the play proceeds to the next stage. Bala and Goyal (2000a) focus on the speed of convergence and on the architecture of the outcome networks.

Experimental studies on the unilateral connections model are performed by Falk and Kosfeld (2003) and by Goeree et al. (2009), among others. For a survey of experimental work on models of network formation, we refer to Kosfeld (2004).

\section{Models with link investments}

In the models we discussed so far, agents have binary choices: to choose a link or not. Several models are studied where agents not only choose which links they want to form, but also how much they want to invest in those links.

Bloch and Jackson (2007) propose a model where each agent proposes investments in each adjacent link. These investments can be positive (offers) or negative (demands). If the total investment in a link is non-negative, then this link will be formed. Each agent receives a utility value given the outcome network, and he pays his proposed investments in the links of the outcome network. Bloch and Jackson (2007) also consider a setting where each agent can also make offers (no demands) to links where he is not adjacent to.

Bloch and Dutta (2009) study a model where each agent has a fixed resource which he has to allocate over his adjacent links. The total amount of allocation on a link determines the strength of this link, which can be interpreted as its reliability. The outcome network yields a payoff for each agent. Bloch and Dutta (2009) focus on the architecture of efficient networks (i.e. for which the sum of all payoffs is maximal) and equilibrium networks. A similar model, but with directed links, is studied by Rogers (2006).

\section{Applications}

Several applications of the models we discussed are examined in the literature. Jackson and Wolinsky (1996) introduce the co-author model in which agents are researchers. Two researchers are linked if they are working together on a project. The synergy of this cooperation depends on the number of others projects where the researchers are involved in. This co-author model is a typical example of the bilateral connections model, since both researchers have to agree on working together.

A typical example of the unilateral connections model is the telephone example given by Bala and Goyal (2000a b). The caller initiates a call, i.e. he pays the telephone company, but both agents can exchange information.

Many applications of endogenous network formation concern oligopoly markets. Here, the agents represent the firms of an oligopoly. A link between two firms is equivalent to some relationship between them, e.g. a collaboration or an agreement. Belleflamme and Bloch (2004) propose a model where a link between two firms means that they do not sell products on the market of the other. In the models studied by 
Goyal and Moraga-Gonzalez (2001), Goyal and Joshi (2003), and Zirulia (2006), a link represents a synergy between two firms. By this synergy, they are able to obtain lower production costs. Goyal and Joshi (2006), and Furusawa and Konishi (2005, 2007) study a network formation game where the agents represent countries, and where two countries can only trade goods without tariffs if they are linked.

Besides social and economic networks, also supply networks can be endogenous. Bergantiños and Lorenzo (2004) study a real-life situation where villagers, who had insufficient water supply, had to form pipelines in order to connect their houses to the water supply network. Observe that this application slightly differs from the unilateral connections model, since the agents are only interested in being connected to a source node, rather than to each other.

\subsection{Outline of this thesis}

This thesis is organized as follows. In Chapters 2 to 7 , we study unilateral network formation. The models that we examine are based on the unilateral connections model.

In Chapter 2 we study the one-way flow model. We provide two different proofs for the existence of Nash networks for games with heterogeneous non-negative profits and owner-homogeneous link costs, i.e. links that belong to the same owner have equal costs. For the case where link costs are heterogeneous, we show by means of a counterexample that Nash networks do not always exist. We provide conditions under which Nash networks do exist. Further, we characterize the architecture of (strict) Nash networks. We also study games where the profits may be negative, and we show that for these games, Nash networks do not always exist. Finally we provide an overview of results regarding the one-way flow model.

We study the complexity of finding best responses in the one-way flow model in Chapter 3. A best response of agent $i$ is a set of own links which gives him a maximal payoff, given the links of the other agents. We show that the problem of finding a best response is computationally intractable (NP-hard), even if link costs are ownerhomogeneous. Further, we provide an algorithm for finding best responses. We study the time- and space-complexity of this algorithm, and its average case performance. Finally, we propose a polynomial-time algorithm for finding a best response in the two-way flow model.

In Chapter 4 we study a model of unilateral network formation which is inspired by the one-way model. We introduce local actions, which are: adding, removing, and replacing an own link, and doing nothing. Global actions are defined as changing the whole set of own links at once. We propose a framework of axiomatic payoff properties that are oriented on local actions and inspired by the one-way flow model. For payoff functions that satisfy these properties, we prove the existence of local-Nash networks, i.e. networks where no agent can improve by playing a local action. Moreover, we show that the derived local-Nash networks are also global-Nash (i.e. networks where 
no agent can improve by playing a global action) for payoff functions that satisfy three properties. Thus, our local approach enables us to prove the existence of global-Nash networks. We show that the properties are independent from each other. Further, we relate them to the payoff functions of the one-way flow model and we provide examples of other payoff functions that satisfy them.

In Chapter 5 we study a dynamic game based on the model of Chapter 4. Starting with an arbitrary initial network, agents iteratively play local actions. We provide examples of how this game is played. We show that, whenever all payoff properties from Chapter 4 are satisfied and agents play good local responses (i.e. improving or payoff-equivalent actions), within a finite number of iterations this procedure reaches a network that no agent wants to modify. The obtained network is local-Nash and moreover, also global-Nash.

In Chapter 6 we provide a full characterization of payoff functions from the one-way flow model that satisfy our framework of properties from Chapter 4.

In Chapter 7 we generalize the two-way flow model. We develop axiomatic payoff properties in a way similar to the approach in Chapter 4 with respect to the one-way flow model. We generalize a proof by Haller et al. (2007) where we make use of our axiomatic payoff properties. We provide a full characterization of payoff functions from the two-way flow model that satisfy these properties, and we provide examples of payoff functions that do not belong to the two-way flow model even though they satisfy all properties.

In Chapter 8 we examine project support games which are inspired by the model of network formation proposed by Bloch and Jackson (2007). Here one can think of a project, consisting of small tasks, that needs to be financed by a set of agents. The agents propose investments in each task. We study which investments are stable, i.e. which investments lead to the financing of the project such that there is no incentive to deviate. We provide an economic example in which project support games can be applied. Further, we relate stability to the Nash equilibrium concept. Finally, we relate the model to other literature in this field.

In Chapter 9, we conclude with a discussion of the axiomatic frameworks proposed in Chapters 4 and 7. We relate those frameworks with two extensions of the unilateral connections model: information decay and imperfectly reliable links. Finally, we provide recommendations for further research.

An overview of the notations and definitions that we use, is provided on page 125 and 126 . 


\section{Chapter 2}

\section{Nash networks in the one-way flow model}

In social and economic networks, agents (e.g. persons or organizations) are able to share valuable information. We study the formation of these networks by modeling it as a non-cooperative game. Links are directed in our game model. Simultaneously and independently, each agent forms a set of links pointing at him. The links of all agents together define the outcome network, which yields a payoff for each agent.

In this chapter, we focus on specific payoff functions, which consist of a cost and a profit part. Each agent pays a certain cost for each formed link. Further, each agent $i$ receives a certain profit (e.g. information) from each other agent $j$, if a directed path from $j$ to $i$ exists. In other words, the direction of the links in the outcome network corresponds to the flow of profits. For this reason, the model of network formation with this payoff structure, is called the one-way flow model.

A network is called a Nash network if no agent can gain a strictly higher payoff by deviating from his set of formed links. In reality, network formation is a dynamic process. But since we focus on Nash networks in this chapter, we model network formation as a static game.

The one-way flow model is proposed by Bala and Goyal (2000a). They characterize and prove the existence of Nash networks for games where profits and link costs are homogeneous, i.e. all profits are equal and all links are equally expensive. Galeotti (2006) studies heterogeneous profits and link costs and he characterizes the architecture of strict Nash networks for various settings while assuming such Nash networks exist.

In this chapter we provide two different proofs for the existence of Nash networks for games with heterogeneous profits and owner-homogeneous link costs, i.e. links that belong to the same owner have equal costs. The first proof is by induction; to show that a Nash network exists for a game with $n$ agents, we will use the induction hypothesis that a Nash network exists for each game with less than $n$ agents. The second proof is 
based on the approach by Billand et al. (2008). In short, the idea behind this approach is that a sequence of networks is constructed such that each successive network is at least as good for each agent. We provide an alternative and easy accessible proof based on this idea.

We show by means of a counterexample that Nash networks may not exist for games where link costs are heterogeneous, even though they are arbitrarily close to the situation of owner-homogeneity. We provide conditions on the profits and link costs under which Nash networks do exist.

Further, we study the architecture of Nash networks. Bala and Goyal (2000a) show that for games with homogeneous link costs and profits, a Nash network is either empty or minimal strongly connected. In this chapter, we provide a full characterization of Nash network architectures for games with various settings of heterogeneity among link costs and profits. We show that Nash networks are fully characterized as minimal networks when link costs are heterogeneous. Further, we show that when link costs are owner-homogeneous, Nash networks also have the property that for each agent with at least two outgoing links, each of these links is contained in a cycle.

We also study the existence and architecture of strict Nash networks, i.e. networks where no agent can gain a higher or equal payoff by deviating. For homogeneous and strictly positive link costs, Bala and Goyal (2000a) prove the existence of strict Nash networks. By means of a counterexample, we show that strict Nash networks do not always exist for games with owner-homogeneous link costs. As for the architecture of strict Nash networks, we provide an overview of the results obtained in the literature.

Finally, we extend this model by allowing negative profits. The interpretation of negative profits is that an agent may not want to be connected to a specific other agent, even if there is no cost for this connection. This extension can be very realistic in network formation. For instance, there are websites on the World Wide Web that you want to avoid, or there may be a person in a friendship network you do not like. We show by means of a counterexample that the existence of Nash networks for games with negative profits is not guaranteed. Further, we characterize Nash networks for these games as minimal networks.

This chapter is outlined as follows. First, in Section 2.1, we introduce the model and provide notations that we will use throughout this chapter. These notations will also be used in other chapters of this thesis. In Section 2.2 we study the existence of Nash networks. For games with owner-homogeneous link costs, we provide two different proofs of existence. For games with heterogeneous link costs, we provide a counterexample, and conditions for which Nash networks do exist. Section 2.3 deals with the architecture of Nash networks. We provide a full characterization of network architectures for games with heterogeneous link costs as well as for games with ownerhomogeneous link costs. In Section 2.4 we examine the existence and architecture of strict Nash networks. In Section 2.5 we study games with possibly negative profits. Finally, in Section 2.6 we provide an overview of the results obtained in the literature and in this chapter with respect to Nash networks in the one-way flow model. 
This chapter is partly based on Derks et al. (2008a) and Derks and Tennekes (2008).

\subsection{Model and notations}

Let $N=\{1, \ldots, n\}$ denote a finite set of agents. We define a network $g$ on the agent set $N$ as a set of links $g \subseteq N \times N$, where loops are not allowed, i.e. $(i, i) \notin g$ for all $i \in N$. We formally define a network as a directed graph, but since we study network formation, we refer to it as a network 1

For convenience we will use the symbols ' + ' and '-' for the union respectively the set exclusion of two networks, or of a network and a single link. In case of ambiguity, these operations are applied from left to right. For instance, the notation $g-g^{\prime}+(j, i)$ stands for $\left(g \backslash g^{\prime}\right) \cup\{(j, i)\}$.

A directed path from $j$ to $i$ in $g$ is a sequence of distinct agents $i_{1}, i_{2}, \ldots, i_{k}$ with $k \geq 1$, such that $j=i_{1}, i=i_{k}$ and $\left(i_{s}, i_{s+1}\right) \in g$ for each $s=1,2, \ldots, k-1$. Notice that for $k=1$ we have that $i=i_{1}$ is a trivial directed path without links from $i$ to himself. An undirected path is defined analogously, but here, either $\left(i_{s}, i_{s+1}\right)$ or $\left(i_{s+1}, i_{s}\right)$ is contained in $g$ for each $s=1,2, \ldots, k-1$. A cycle is defined in the same way as a directed path with $k \geq 2$, and with the exception that $i_{1}=i_{k}$. Thus, a cycle is always directed, unless mentioned otherwise. Let the outdegree of agent $i$ in network $g$ be defined as the number of outgoing links of $i$ in $g$.

Agent $j$ is called active in $g$ if $j$ is a begin- or endpoint of a link in $g$. We define $g^{j}$ for agent $j$ to be the following subset of $g$ :

$$
g^{j}=\{(k, \ell):(k, \ell) \in g \text {, and an undirected path between } \ell \text { and } j \text { exists in } g\},
$$

and we refer to $g^{j}$ as the component of $g$ where $j$ is active. Observe that $g^{j}$ is empty in case $j$ is isolated (i.e. not active) in $g$. Subset $g^{\prime}$ is a called a component if an agent $j \in N$ exists such that $g^{\prime}=g^{j}$.

Let the set of agents that $i$ observes in network $g$ be defined as

$$
N_{i}(g)=\{j \in N: \text { a directed path from } j \text { to } i \text { exists in } g\}
$$

and let

$$
N_{i}^{d}(g)=\{j:(j, i) \in g\} .
$$

Note that $i \in N_{i}(g)$, and $i \notin N_{i}^{d}(g)$.

For each agent $i$, let $\pi_{i}: \mathcal{G} \rightarrow \mathbb{R}$ be a payoff function, where $\mathcal{G}$ is the set of all possible networks on $N$. Let $\pi=\left(\pi_{i}\right)_{i \in N}$ be a joint payoff function. We will use the following class of payoff functions, which is proposed by Galeotti (2006):

$$
\pi_{i}(g)=\sum_{j \in N_{i}(g)} v_{i j}-\sum_{j \in N_{i}^{d}(g)} c_{i j} \quad \forall i \in N .
$$

\footnotetext{
${ }^{1}$ In graph theory, it is common to define a directed graph as a set of nodes and a set of arcs. Since in our case the set of agents is fixed, it is left out of the definition of a network.
} 
Here $v_{i j}$ is the profit that agent $i$ receives from observing $j$ and $c_{i j}$ is the cost of link $(j, i)$ for agent $i$. The profits and costs are assumed to be non-negative throughout this chapter, except for Section 2.5 in which we study games where profits may be negative. We refer to these payoff functions as B\&G-1 functions, since Bala and Goyal (2000a) introduced the one-way flow model.

We follow other literature on one-way flow models in the sense that the direction of the links indicates information flow. Consequently link $(j, i)$, which is depicted from $j$ to $i$, is owned and paid by agent $i$.

We say that link costs are homogeneous if there is a constant $c$ with $c_{i j}=c$ for all $i, j \in N$. We say that link costs are owner-homogeneous if for each agent $i$ there is a constant $c_{i}$ with $c_{i j}=c_{i}$ for all $j \in N$. Otherwise, link costs are heterogeneous. These definitions also apply to the profits.

In this chapter we study a non-cooperative game. This game is played by the agents in $N$. Simultaneously and independently, each agent $i$ chooses a possibly empty set $S$ of agents he wants to connect to by creating the links $(j, i)$, for each $j \in S$. Together, the links of all agents form a network $g \in \mathcal{G}$. Then, each agent $i$ receives a payoff $\pi_{i}(g)$. Since each agent wants to maximize his payoff in response to what the other agents are doing, the focus of this chapter is on Nash networks, i.e. networks in which no agent can gain from a unilateral deviation.

We define an action of agent $i$ by a set of agents $S \subseteq N \backslash\{i\}$. The network that results after $i$ chooses to link up with the agents in $S$ w.r.t. network $g$, is described by

$$
g_{-i}+\{(j, i): j \in S\}
$$

with $g_{-i}$ denoting the network obtained from $g$ after removing the links $(j, i) \in g$ owned by $i$. An action $S^{*}$ of agent $i$ is called a best response with respect to $\pi_{i}$ and $g$ if

$$
\pi_{i}\left(g_{-i}+\left\{(j, i): j \in S^{*}\right\}\right) \geq \pi_{i}\left(g_{-i}+\{(j, i): j \in S\}\right)
$$

for all actions $S \subseteq N \backslash\{i\}$.

A network $g$ is a Nash network if $N_{i}^{d}(g)$ is a best response for all $i \in N$, i.e. if for each agent $i \in N$

$$
\pi_{i}(g) \geq \pi_{i}\left(g_{-i}+\{(j, i): j \in S\}\right)
$$

for all actions $S \subseteq N \backslash\{i\}$.

A network $g$ is a strict Nash network if $N_{i}^{d}(g)$ is the unique best response for each agent $i \in N$.

\subsection{Existence of Nash networks}

In this section we provide two different proofs for the existence of Nash networks for games with owner-homogeneous link costs. The first proof (Section 2.2.1) is by induction and the second one (Section 2.2.2) by construction. A third proof is provided 
in Chapter 4, where the existence of Nash networks is proved for a more general class of payoff functions.

In Section 2.2.3, we study games with heterogeneous link costs. We provide an example for which Nash networks do not exist, even though link costs are arbitrarily close to the situation of owner-homogeneity. Furthermore, we provide conditions on the profits and link costs under which Nash networks do exist.

\subsubsection{Proof by induction}

It is common in the literature to consider the set of agents, the costs, and the profits as fixed. However, our approach requires the comparison of different game situations. To facilitate this approach we define a (non-cooperative) one-way flow game to be a triple $(N, v, c)$ on agent set $N$, with profits $v=\left(v_{i j}\right)_{i, j \in N}$ and $\operatorname{costs} c=\left(c_{i j}\right)_{i, j \in N, i \neq j}$ that define B\&G-1 functions $\pi_{i}, i \in N$, as described in 2.2.

An agent $k$ with no own links in a network $g$ is only of interest for those agents $i$ with $c_{i k} \leq v_{i k}$, since

$$
\begin{aligned}
\pi_{i}(g+(k, i)) & =\sum_{j \in N_{i}(g+(k, i))} v_{i j}-\sum_{j \in N_{i}^{d}(g+(k, i))} c_{i j} \\
& =\sum_{j \in N_{i}(g)} v_{i j}+v_{i k}-\sum_{j \in N_{i}^{d}(g)} c_{i j}-c_{i k} \\
& =\pi_{i}(g)+v_{i k}-c_{i k} .
\end{aligned}
$$

For costs sufficiently small, the so-called cycle networks are Nash networks. Cycle networks consist of one cycle joining all agents (see Figure 2.1).

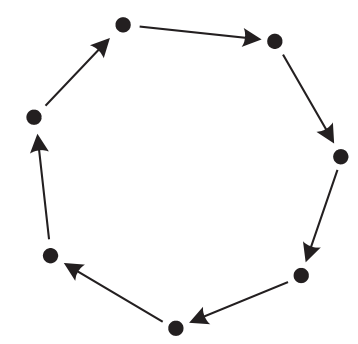

Figure 2.1: Cycle network.

Lemma 2.2.1. For an owner-homogeneous cost one-way flow game $(N, v, c)$, with

$$
c_{i} \leq \sum_{j \in N, j \neq i} v_{i j} \quad \text { for all } i \in N
$$

all cycle networks are Nash networks. 
Proof. Let $g$ be a cycle network.

Each agent $i$ obtains $\pi_{i}(g)=\sum_{j \in N} v_{i j}-c_{i}$. If agent $i$ plays $\emptyset$ then he obtains $v_{i i}$, which is at most $\pi_{i}(g)$ by (2.4). If he plays a non-empty action, his payoff will be at most $\pi_{i}(g)$, because he already receives maximal profits in $g$ (since profits are non-negative), with only one own link. Therefore, $g$ is a Nash network.

Observe that non-negativity of the profits is required for Lemma 2.2.1. In Example 2.5.1 of Section 2.5 we examine a game with negative profits where no cycle network is Nash, although condition 2.4 is satisfied.

In the owner-homogeneous cost case, we also observe the following: if link $(j, k)$ is present in $g$, then linking up with agent $k$ is at least as good for an agent $i \neq j, k$, as linking up with $j$ :

$$
\pi_{i}(g+(k, i)) \geq \pi_{i}(g+(j, i)) \quad \text { whenever }(j, k) \in g .
$$

In the next theorem, we prove the existence of Nash networks for games with ownerhomogeneous link costs. This proof is based on induction, but constructive in nature. Either any cycle network constitutes a Nash network or there is an agent who is not interested in being 'involved'.

In the latter case, there may be an agent $i$ who is interested in linking up with this uninvolved agent. In that case the profit values are adapted as described in the proof, and a Nash network is searched in the situation without the uninvolved agent; next, this network is extended by connecting the uninvolved agent with agent $i$.

The case where no agent is interested in linking up with this uninvolved agent is somewhat simpler, as any Nash network on the set of agents excluding the uninvolved agent is also a Nash network on the full set of agents.

Theorem 2.2.2. Nash networks exist for any one-way flow game with owner-homogeneous costs.

Proof. We will prove the theorem by induction. One-way flow games with only one agent trivially have a Nash network. Suppose that $(N, v, c)$ with $N=\{1,2, \ldots, n\}$ is an owner-homogeneous cost one-way flow game that does not have a Nash network, while all one-way flow games with less than $n$ agents do have Nash networks. According to Lemma 2.2.1, this implies that there is an agent $i$ with $c_{i}>\sum_{j \in N, j \neq i} v_{i j}$.

Without loss of generality assume $i=n$. Observe that the best response of agent $n$ in any network is the empty set. Consider the owner-homogeneous cost one-way flow game $\left(N^{\prime}, v^{\prime}, c^{\prime}\right)$, with $N^{\prime}=N \backslash\{n\}$, and $v^{\prime}$ and $c^{\prime}$ equal to $v$ and $c$ restricted to the agents in $N^{\prime}$. Let $\pi_{i}^{\prime}$ denote the B\&G-1 function for agent $i$ in $\left(N^{\prime}, v^{\prime}, c^{\prime}\right)$. It is clear that $\pi_{i}^{\prime}(g)=\pi_{i}(g)$ for each network $g$ on $N^{\prime}$, and $i \neq n$.

Since $N^{\prime}$ has $n-1$ agents, $\left(N^{\prime}, v^{\prime}, c^{\prime}\right)$ has a Nash network, say $g^{\prime}$. Consider $g^{\prime}$ as a network on $N$, and recall the assumption that $(N, v, c)$ does not have a Nash network. Therefore, there is an agent $k$ in $(N, v, c)$ who does not play his best response in $g^{\prime}$. Of course $k \neq n$, as $N_{n}^{d}\left(g^{\prime}\right)=\emptyset$ is obviously his best response. Let $T \subseteq N \backslash\{k\}$ be a best 
response of $k$ in $g^{\prime}$, and suppose $n \notin T$. Then $\left(g^{\prime}\right)_{-k}+\{(j, k): j \in T\}$ is a network in $N^{\prime}$ so that

$$
\begin{aligned}
\pi_{k}^{\prime}\left(g^{\prime}\right) & =\pi_{k}\left(g^{\prime}\right) \\
& <\pi_{k}\left(\left(g^{\prime}\right)_{-k}+\{(j, k): j \in T\}\right) \\
& =\pi_{k}^{\prime}\left(\left(g^{\prime}\right)_{-k}+\{(j, k): j \in T\}\right),
\end{aligned}
$$

which is a contradiction with $g^{\prime}$ being a Nash network for $\left(N^{\prime}, v^{\prime}, c^{\prime}\right)$.

Now suppose that $n \in T$. Without loss of generality assume $k=1$. From $n \in T$ and $N_{n}^{d}\left(g^{\prime}\right)=\emptyset$ we conclude that $c_{1} \leq v_{1 n}$ must hold (see $2.3 p$ ). Consider the following adapted profits $v^{*}=\left(v_{i j}^{*}\right)_{i, j \in N^{\prime}}$ :

$$
v_{i j}^{*}= \begin{cases}v_{i j} & \text { if } j \neq 1, \\ v_{i 1}+v_{i n} & \text { if } i \neq 1, j=1, \\ v_{11}+v_{1 n}-c_{1} & \text { if } i, j=1 .\end{cases}
$$

Observe that these values are non-negative. Let $\pi_{i}^{*}$ denote the B\&G-1 functions in $\left(N^{\prime}, v^{*}, c^{\prime}\right)$. The profits $v_{i j}^{*}$ are chosen such that $\pi_{i}^{*}(g)=\pi_{i}(g+(n, 1))$ holds for all networks $g$ on $N^{\prime}$, and for all $i \in N^{\prime}$.

By assumption, the one-way flow game $\left(N^{\prime}, v^{*}, c^{\prime}\right)$ has a Nash network, say $g^{*}$; since $(N, v, c)$ does not have a Nash network, there is an agent $i$ in $N$ who can improve in the network $g^{*}+(n, 1)$, in the context of the game $(N, v, c)$, say by choosing the links with the agents in $S \subseteq N \backslash\{i\}$. This agent is not $n$ because $N_{n}^{d}\left(g^{*}+(n, 1)\right)=\emptyset$.

Suppose $i \neq 1$. If $n \in S$, then according to 2.5, the action $S \backslash\{n\} \cup\{1\}$ is at least as good as $S$; therefore we may assume $n \notin S$. The resulting network $\left(g^{*}+(n, 1)\right)_{-i}+$ $\{(j, i): j \in S\}$, after $i$ performs the improvement, yields a higher payoff for agent $i$. Then

$$
\begin{aligned}
\pi_{i}^{*}\left(g^{*}\right) & =\pi_{i}\left(g^{*}+(n, 1)\right) \\
& <\pi_{i}\left(\left(g^{*}+(n, 1)\right)_{-i}+\{(j, i): j \in S\}\right) \\
& =\pi_{i}\left(\left(g_{-i}^{*}+\{(j, i): j \in S\}\right)+(n, 1)\right) \\
& =\pi_{i}^{*}\left(g_{-i}^{*}+\{(j, i): j \in S\}\right) \\
& \leq \pi_{i}^{*}\left(g^{*}\right),
\end{aligned}
$$

where the second inequality holds because of $g^{*}$ being a Nash network for $\left(N^{\prime}, v^{*}, c^{\prime}\right)$. Thus, we arrived at a contradiction, so that we must have $i=1$.

Due to $c_{1} \leq v_{1 n}$, and agent $n$ having no own links, we may assume $n \in S$ (see the observation concerning (2.3)). Then

$$
\begin{aligned}
\pi_{1}^{*}\left(g^{*}\right) & =\pi_{1}\left(g^{*}+(n, 1)\right) \\
& <\pi_{1}\left(g_{-1}^{*}+\{(j, 1): j \in S\}\right) \\
& =\pi_{1}\left(g_{-1}^{*}+\{(j, 1): j \in S \backslash\{n\}\}+(n, 1)\right) \\
& =\pi_{1}^{*}\left(g_{-1}^{*}+\{(j, 1): j \in S \backslash\{n\}\}\right) \\
& \leq \pi_{1}^{*}\left(g^{*}\right) ;
\end{aligned}
$$


a contradiction. We conclude that $(N, v, c)$ must have a Nash network.

Observe that the Nash networks we obtain have at most one cycle, if the Nash networks excluding the uninvolved agent also have at most one cycle. The same applies when considering networks where agents have an outdegree of at most one. The following corollary is now easily established.

Corollary 2.2.3. Nash networks exist, with at most one cycle and maximum outdegree of at most 1, for any one-way flow game with owner-homogeneous costs.

There may also exist Nash networks with multiple cycles and with outdegrees higher than 1. Consider the following example.

Example 2.2.4. Let $n=7$, and let $c_{i j}=1$ and $v_{i j}=1$ for all $i, j \in N$. Consider the network with two cycles that is depicted in Figure 2.2 .

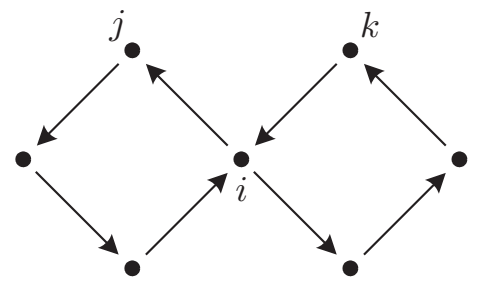

Figure 2.2: A Nash network with two cycles.

Notice that agent $i$ has two outgoing links. It can be verified that this network is a Nash network. Observe that this network is not strict Nash, since $j$ can replace $(i, j)$ by $(k, j)$, which yields the same payoff.

We revisit this example in Section 2.4 where we examine the existence and architecture of strict Nash networks. In Section 2.3 we examine the architecture of Nash networks.

\subsubsection{Proof by construction}

Consider the transformation $\pi_{i}^{\prime}(g)=\pi_{i}(g)-v_{i i}$. Since this transformation has no influence on the strategic behavior of agent $i$, we may assume that $v_{i i}=0$.

Let $g_{-i j}=g_{-i}^{j}+(j, i)$, where $g_{-i}^{j}$ means $\left(g_{-i}\right)^{j}$, i.e. the component of $g_{-i}$ where $j$ is active. By the non-negativity of the profits (and $v_{i i}=0$ ), it is immediate that

$$
\pi_{i}(g) \leq \pi_{i}(g-(j, i))+\pi_{i}\left(g_{-i j}\right) \quad \text { for all }(j, i) \in g .
$$

Therefore, when agent $i$ plays a best response then $\pi_{i}\left(g_{-i j}\right) \geq 0$ for each $j \in N_{i}^{d}(g)$. We say that link $(j, i)$ is beneficial in $g$ when $\pi_{i}\left(g_{-i j}\right) \geq 0$, and a network $g$ is called beneficial when all its links are beneficial. Observe that a Nash network is beneficial. 
Let a network be proper if the outdegree of each agent is at most 1 . In a proper network $g$, an agent $i$ observes each agent $j \in N_{i}(g)$ via a unique directed path, and by this observation it is not hard to see that equality holds in (2.6), whenever $g$ is proper.

For any network $g$ let $C(g)$ denote the set of agents located on a cycle in $g$. Further, let $D(g)$ denote the set of agents who observe at least one agent in $C(g)$, i.e. $D(g)=$ $\left\{i: N_{i}(g) \cap C(g) \neq \emptyset\right\}$.

Assuming that the agents are numbered $1,2, \ldots, n$ there is a unique ordering $s_{1}$, $s_{2}, \ldots, s_{d}$ of agents in $D(g)$, with $s_{k-1}<s_{k}$ for each $k=2, \ldots, d=|D(g)|$. Consider the following network $\hat{g}$, defined by $\hat{g}=g$ in case $d=0$, and for $d \geq 2$ (notice that $d$ cannot be 1 , since a cycle contains at least two agents) defined by:

$$
\hat{g}=\left\{\left(s_{1}, s_{2}\right), \ldots,\left(s_{d-1}, s_{d}\right),\left(s_{d}, s_{1}\right)\right\}+\{(j, i) \in g: j \notin D(g)\} .
$$

An example of this network is depicted in Figure 2.3 .

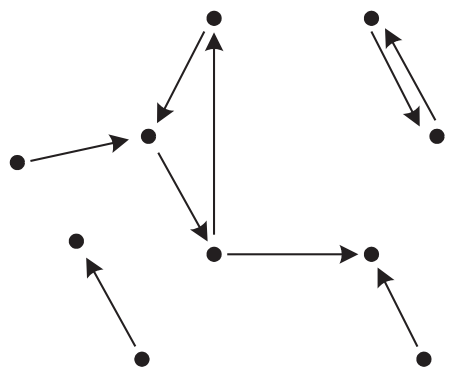

$g$

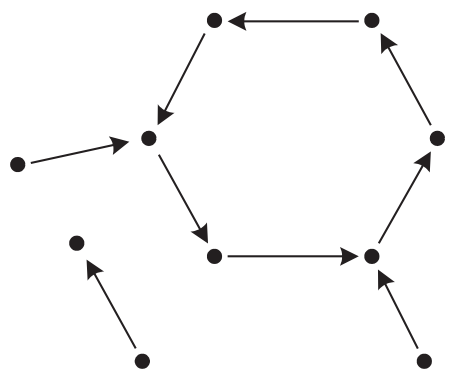

$\hat{g}$

Figure 2.3: Network $g$ with the corresponding network $\hat{g}$.

Lemma 2.2.5. If for a network $g$ the agents outside $D(g)$ have at most one outgoing link then $\hat{g}$ is proper.

Proof. Each agent in $D(g)$ has exactly one outgoing link in $\hat{g}$, namely the one on the cycle. Each agent outside $D(g)$ has the same outdegree in $\hat{g}$ as in $g$.

Naturally, we set $\hat{g}=g$ whenever $D(g)=\emptyset$, i.e. whenever there are no cycles in $g$. Otherwise, $\hat{g}$ has exactly one cycle. Notice also that an agent owns no more links in $\hat{g}$ than in $g$, so that in the owner-homogeneous costs situation the agents in $\hat{g}$ face costs at most as in $g$. Furthermore, the agents observe the same or more agents in $\hat{g}$ :

Lemma 2.2.6. $N_{i}(g) \subseteq N_{i}(\hat{g})$ for each agent $i$.

Proof. Let $j \in N_{i}(g)$. 
Suppose $i \notin D(g)$, then any directed path from $j$ to $i$ in $g$ does not visit an agent in $D(g)$, and therefore it is also a directed path in $\hat{g}$. This implies that $i$ observes $j$ in $\hat{g}: j \in N_{i}(\hat{g})$.

Now suppose $i \in D(g)$. If also $j \in D(g)$ then $i$ observes $j$ via the cycle in $\hat{g}$. So, let $j \notin D(g)$, and let $k$ be the first agent in $D(g)$ on a directed path from $j$ to $i$ in $g$. Then, the directed subpath from $j$ to $k$ is also a directed path in $\hat{g}$, implying $j \in N_{k}(\hat{g})$. Since $k \in N_{i}(\hat{g})$, we have $j \in N_{i}(\hat{g})$.

Lemma 2.2.7. Let $\pi$ be a joint BEG-1 function with owner-homogeneous link costs. If a network $g$ is beneficial, then network $\hat{g}$ is also beneficial.

Proof. Let $g$ be beneficial, and let $(j, i) \in \hat{g}$. We prove that $(j, i)$ is beneficial in $\hat{g}$.

Suppose $j \notin D(g)$. This implies $(j, i) \in g$. For $k \in N_{i}\left(g_{-i j}\right)$ there is a directed path from $k$ to $i$ via link $(j, i)$ in $g$, and none of the visited agents are members of $D(g)$ (except possibly $i$ ), so that this path is also present in $\hat{g}_{-i j}$. This implies $k \in N_{i}\left(\hat{g}_{-i j}\right)$, and therefore,

$$
\pi_{i}\left(\hat{g}_{-i j}\right)=\sum_{k \in N_{i}\left(\hat{g}_{-i j}\right)} v_{i k}-c_{i} \geq \sum_{k \in N_{i}\left(g_{-i j}\right)} v_{i k}-c_{i}=\pi_{i}\left(g_{-i j}\right) .
$$

Beneficiality of link $(j, i)$ in $g$ therefore implies the beneficiality of $(j, i)$ in $\hat{g}$.

Now, suppose $j \in D(g)$. Then the link $(j, i)$ is a link of the cycle of $\hat{g}$. It is evident that $D(g) \subseteq N_{i}\left(\hat{g}_{-i j}\right)$. Also, $D(g) \cap N_{i}^{d}(g) \neq \emptyset$, say $k \in D(g) \cap N_{i}^{d}(g)$. We know that $(k, i)$ is beneficial in $g$. Since link costs are owner-homogeneous, i.e. $c_{i k}=c_{i j}$, we conclude that link $(j, i)$ is beneficial in $\hat{g}$ whenever

$$
N_{i}\left(g_{-i k}\right) \subseteq N_{i}\left(\hat{g}_{-i j}\right)
$$

So, let $r \in N_{i}\left(g_{-i k}\right)$. If $r \in D(g)$ then also $r \in N_{i}\left(\hat{g}_{-i j}\right)$ as we mentioned before. Thus, assume $r \notin D(g)$. There is a directed path from $r$ to $k$ in $g_{-i}$. Let $r^{\prime}$ be the first agent from $D(g)$ on this path. Then the directed subpath from $r$ to $r^{\prime}$ is present in $\hat{g}$. Extend this path with the directed path from $r^{\prime}$ to $j$ on the cycle of $\hat{g}$, thus obtaining a directed path from $r$ to $j$ in $\hat{g}$. This path does not visit agent $i$ since $i$ is not on the directed path from $r$ to $r^{\prime}$, and also $i$ is not located on the cycle between $r^{\prime}$ and $j$ since $i$ is found on the cycle right after agent $j$. Hence, $r \in N_{i}\left(\hat{g}_{-i j}\right)$, implying (2.7). We conclude that $(j, i)$ is beneficial in $\hat{g}$.

Theorem 2.2.8. Let $\pi$ be a joint BEG-1 function with owner-homogeneous link costs. Then, the following procedure terminates in a finite number of steps at a proper Nash network.

Step 0: Let $t=1$, and $g_{t}=\emptyset$;

Step 1: If $g_{t}$ is Nash then STOP. 
Step 2: Let agent $i$ perform a best response in $g_{t}$, with as many links as possible and, in case of indifference, with as many observed agents as possible, and let $g_{t+1}$ be the resulting network;

Step 3: Let $g_{t+2}=\widehat{g_{t+1}}$;

Step 4: Let $t=t+2$, and return to Step 1 .

Proof. Let $i$ be an agent who applies a best response $B$ to $g_{t}$ with as many links as possible and, in case of indifference, with as many observed agents as possible, obtaining $g_{t+1}$. Suppose that $g_{t}$ is proper and beneficial. We prove that (i) $N_{i}\left(g_{t+1}\right) \supset N_{i}\left(g_{t}\right)$, (ii) $g_{t+2}$ is beneficial, and (iii) $g_{t+2}$ is proper.

(i) Let $j \in N_{i}^{d}\left(g_{t}\right)$, and suppose $j \notin B$. Since $g_{t}$ is proper it follows that agent $j$ is the unique agent who observes all agents in $N_{j}\left(\left(g_{t}\right)_{-i}^{j}\right)$. This shows that whenever $N_{j}\left(\left(g_{t}\right)_{-i}^{j}\right) \cap B \neq \emptyset$, then $j \in B$. Since we assumed $j \notin B$, it follows that $N_{j}\left(\left(g_{t}\right)_{-i}^{j}\right) \cap B=\emptyset$, so that

$$
\pi_{i}\left(g_{t+1}+(j, i)\right)=\pi_{i}\left(g_{t+1}\right)+\pi_{i}\left(\left(g_{t+1}\right)_{-i j}\right)=\pi_{i}\left(g_{t+1}\right)+\pi_{i}\left(\left(g_{t}\right)_{-i j}\right) \geq \pi_{i}\left(g_{t+1}\right) .
$$

The latter inequality is due to the beneficiality of the links in $g_{t}$. Since the best response $B$ is chosen as large as possible, and the action $B \cup\{j\}$ is at least as good as $B$, we arrive at a contradiction. Hence,

$$
N_{i}^{d}\left(g_{t}\right) \subseteq B
$$

Since $B$ is a strict improvement, we have $N_{i}^{d}\left(g_{t}\right) \subset B$, implying $N_{i}\left(g_{t+1}\right) \supset N_{i}\left(g_{t}\right)$.

(ii) By 2.8 it follows that $g_{t} \subseteq g_{t+1}$, and this proves the beneficiality of the links $(j, \ell) \in g_{t}$ in $g_{t+1}$. As for the added links $(j, i)$, with $j \in B \backslash N_{i}^{d}\left(g_{t}\right)$ we observed already (see (2.6)) that these links are beneficial in $g_{t+1}$. So, $g_{t+1}$ is beneficial, and because of Lemma 2.2 .7 , we conclude that $\widehat{g_{t+1}}=g_{t+2}$ is beneficial.

(iii) To show that $g_{t+2}$ is proper, by Lemma 2.2.5 we only need to check the outdegrees of the agents outside $D\left(g_{t+1}\right)$ in $g_{t+1}$. Only the outdegree of the agents in $B \backslash N_{i}^{d}\left(g_{t}\right)$ are raised by 1 , with respect to the outdegree in the proper network $g_{t}$. Therefore, we only need to check that the agents in $B \backslash\left(N_{i}^{d}\left(g_{t}\right) \cup D\left(g_{t+1}\right)\right)$ have outdegree 0 in $g_{t}$.

Consider an agent $j \in B \backslash N_{i}^{d}\left(g_{t}\right)$, and suppose that his outdegree in $g_{t}$ is 1 , say $(j, k) \in g_{t}$. It follows that a directed path from $k$ to $j$ exists in $g_{t}$, for otherwise $i$ observes more agents by playing $B \backslash\{j\} \cup\{k\}$ than by playing $B$ (agent $k$ is only observed in the first case), which contradicts our assumption in Step 2. Therefore $j$ is necessarily located on the cycle of $g_{t}$, i.e. $j \in D\left(g_{t}\right)$. Observe that $D\left(g_{t}\right)$ is a subset of $D\left(g_{t+1}\right)$, so that we conclude that $j \notin B \backslash\left(N_{i}^{d}\left(g_{t}\right) \cup D\left(g_{t+1}\right)\right)$. Hence, all agents in $B \backslash\left(N_{i}^{d}\left(g_{t}\right) \cup D\left(g_{t+1}\right)\right)$ have outdegree 0 in $g_{t}$. 
Observe that we start the procedure with a beneficial, proper network. By Lemma 2.2.6 and part (i) we conclude that the total number of observed agents of the constructed networks does not decrease, and strictly increases when Step 2 is performed. Therefore, the procedure has to terminate with a network that is Nash. Observe that this network is also beneficial and proper.

\subsubsection{Heterogeneous link costs}

For games with heterogeneous costs, Nash networks do not always exist as we will see in the next example. The link costs in this example can be chosen arbitrarily close to the situation of owner-homogeneity.

Example 2.2.9. Let $N=\{1,2,3,4\}$ be the set of agents, and let $\pi$ be the following joint B\&G-1 function with homogeneous profits and heterogeneous link costs. Let $v_{i j}=1$ for all $i, j$ with $i \neq j$, and let $v_{i i}=0$ for all $i$. The numbers next to the links in Figure 2.4 (a) indicate the costs of these links. Here, $\epsilon$ is a strictly positive number which can be chosen arbitrarily close to 0 . The costs of the links that are not depicted in this figure are the following:

* links directed to agent 1 have cost $1+\epsilon$,

* links directed to agent 2 have cost $2+\epsilon$,

* links directed to agents 3 and 4 have cost $3+\epsilon$,

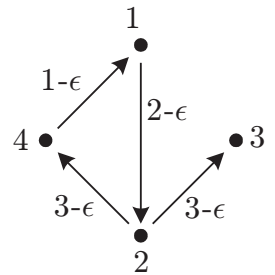

(a)

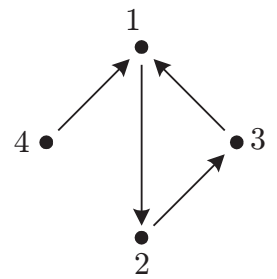

(b)

Figure 2.4: (a) the link costs, and (b) the network obtained when agent 4 plays $\emptyset$ and the other agents play best responses.

The best response of agent 4 to any network is either $\{2\}$ or $\emptyset$, since those are the only actions for which agent 4 may have a non-negative payoff.

First, suppose that agent 4 plays $\{2\}$ as a best response in a Nash network. Consequently, the unique best response of agent 1 is $\{4\}$. Agent 2 has one unique best response to this situation: $\{1\}$. Finally, agent 3 has one unique best response, which is $\{2\}$. The obtained network is the same as depicted in Figure 2.4(a). It follows that $\{2\}$ is not a best response of agent 4 , since $\emptyset$ gives a higher payoff. Hence, there is no Nash network in which agent 4 plays $\{2\}$. 
Now suppose that agent 4 plays $\emptyset$ as a best response in a Nash network. Agent 1 will include 4 in every best response to this situation. Then, the unique best response for agent 2 is $\{1\}$. To this situation, the unique best response of agent 3 is $\{2\}$. Hence, the unique best response of agent 1 is $\{3,4\}$. The obtained network is depicted in Figure 2.4(b). Now agent 4 has a unique best response to this new situation, which is $\{2\}$. This contradicts our assumption of agent 4 playing $\emptyset$ in a Nash network. Hence no Nash networks exist.

Billand et al. (2008) claim that Nash networks always exist if for all $i, j, j^{\prime} \in N$ holds that $\left|c_{i j}-c_{i j^{\prime}}\right|<v_{i}$ (see their Proposition 3). However, in Example 2.2.9. link costs are close to owner-homogeneity, i.e. $\left|c_{i j}-c_{i j^{\prime}}\right| \leq 2 \epsilon$ for all $i, j, j^{\prime} \in N$. Since $v_{i j}=1$ for all $i, j \in N$, we have $\left|c_{i j}-c_{i j^{\prime}}\right|<1=v_{i}$ for $\epsilon<\frac{1}{2}$. Therefore, this example is a counterexample for Proposition 3 in Billand et al. (2008).

Although Nash networks do not always exist when link costs are heterogeneous, there are conditions under which they do exist ${ }^{2}$

Proposition 2.2.10. Let $\pi$ be a joint BEG-1 function. If

$$
\begin{array}{ll}
c_{i j} \leq v_{i j}+c_{i k} & \text { for all } i, j, k \in N \\
c_{i j} \leq \sum_{k \in N, k \neq i} v_{i k} & \text { for all } i, j \in N
\end{array}
$$

then any cycle network is a Nash network.

Proof. Let $g$ be a cycle network. Suppose it is not Nash, say $i$ can improve by playing $\tilde{S}$. Let $\tilde{g}$ be the obtained network. Further, let $S=\{j\}$ be his current action, i.e. link $(j, i)$ is on the cycle in $g$ (see Figure 2.5.

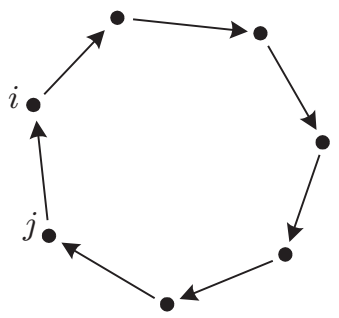

Figure 2.5: Network $g$.

By 2.10 it follows that $\pi_{i}(g) \geq 0$. Since $\tilde{S}$ is a strictly improving action, we conclude that $\tilde{S} \neq \emptyset$.

Suppose that $j \notin \tilde{S}$. Let $k$ be any agent in $\tilde{S}$. Further, let $M=N_{i}(\tilde{g})$ be the set of agents whom $i$ observes in $\tilde{g}$. Clearly, $j \notin M$.

\footnotetext{
${ }^{2}$ These results are obtained from personal communication with S. Sarangi (Louisiana State University, USA), P. Billand, and C. Bravard (Jean Monnet University, France).
} 
Since link costs are non-negative, we have

$$
\begin{aligned}
\pi_{i}(\tilde{g}) & \leq\left(\sum_{\ell \in M} v_{i \ell}\right)-c_{i k} \\
& \leq\left(\sum_{\ell \in M} v_{i \ell}\right)+v_{i j}-c_{i j} \quad \text { by } 2.9 \\
& \leq\left(\sum_{\ell \in N} v_{i \ell}\right)-c_{i j} \\
& =\pi_{i}(g) .
\end{aligned}
$$

This contradicts $\tilde{S}$ being a strict improvement.

Hence $j \in \tilde{S}$. Since $N_{i}\left(g_{-i j}\right)=N$ and link costs are non-negative, we must have $\tilde{S}=\{j\}$; again a contradiction. We conclude that $g$ is a Nash network.

Observe that the B\&G-1 functions of agents 3 and 4 in Example 2.2.9 do not satisfy condition 2.10), because the link cost $3+\epsilon$ exceeds the total profit, which is 3 .

As an alternative for condition 2.10 , consider the following condition:

$$
c_{i j}+c_{i k}>\sum_{r \in N, r \neq i} v_{i r} \quad \text { for all } i, j, k \in N \text {. }
$$

Due to this condition, it is not worthwhile to have more than one own link. Therefore, we can already observe that for joint payoff functions that satisfy 2.11), in any Nash network, each agent has at most one own link. Combining (2.11) with (2.9) enables us to prove the existence of Nash networks:

Proposition 2.2.11. Let $\pi$ be a joint BEG-1 function. If $\pi$ satisfies (2.9) and 2.11) then Nash networks exist where each non-empty component is either a cycle or a directed path.

Proof. Consider a network $g$ where all agents owning a link have a non-negative payoff. Observe that these agents have exactly one link, due to 2.11. Also, assume that each non-empty component of $g$ is either a cycle or a directed path. The empty network fulfills this architecture, so that we may assume that $g$ is chosen to have as many links as possible, i.e., any network with more links contains an agent with negative payoffs or a component which is not a cycle or a directed path.

Now suppose that this network $g$ is not Nash, say agent $i$ can improve. Distinguish two cases:

(A) agent $i$ has a (unique) link in $g$, say $(j, i)$, and

(B) agent $i$ has no links in $g$.

Case A Since $i$ has a non-negative payoff in $g$, and $(j, i)$ is his only link in $g$, it follows that $\pi_{i}\left(g_{-i j}\right) \geq 0$. Using 2.11, we conclude that the improvement of $i$ must be the 
replacement of $(j, i)$ with another link, say $(k, i)$, i.e. $\pi_{i}(g-(j, i)+(k, i))>\pi_{i}(g)$. Observe that

$$
0 \leq \pi_{i}(g)=\pi_{i}\left(g_{-i j}\right)=\pi_{i}\left(g_{-i}+(j, i)\right)<\pi_{i}\left(g_{-i}+(k, i)\right)=\pi_{i}\left(g_{-i k}\right)
$$

Therefore, we have $\pi_{i}\left(g_{-i j}\right)+\pi_{i}\left(g_{-i k}\right)>0$. Because of [2.11), it follows that $\pi_{i}\left(g_{-i}+\right.$ $(j, i)+(k, i))<0$. Therefore, we must have

$$
\pi_{i}\left(g_{-i}+(j, i)+(k, i)\right) \neq \pi_{i}\left(g_{-i j}\right)+\pi_{i}\left(g_{-i k}\right),
$$

and this is only possible if there is an agent $r \in N$ who is observed by agent $i$ both via agent $j$ and agent $k$. This implies that on the paths from $r$ to $j$ and from $r$ to $k$, there is an agent with outdegree greater than 1 , which is in contradiction with the architecture described above. Therefore, case A does not occur.

Case B By 2.11, it follows that agent $i$ 's improvement is the addition of one link, say $(k, i)$. If agent $k$ is active in a component of $g$ that is a directed path, then, due to (2.9), $k$ may be chosen to be the head node of this path. The network

$$
g^{\prime}=g+(k, i)
$$

fulfills the architecture as described previously. Also, all agents receive non-negative payoffs in $g^{\prime}$, since profits are non-negative. This contradicts the fact that $g$ is chosen with maximum number of links with respect to these properties. Therefore, $k$ is active in a cycle component, and there is an agent $r$ with $(k, r) \in g$. The component in $g$, where $i$ is active, is necessarily empty or a directed path, with $i$ being the tail node. Let $j$ be the head node of this path (with $j=i$ in case $g^{i}=\emptyset$ ). Then one can easily show that the network

$$
g^{\prime \prime}=g-(k, r)+(k, i)+(j, r)
$$

fulfills the architecture as described previously. The networks $g$ and $g^{\prime \prime}$ are depicted in Figure 2.6.

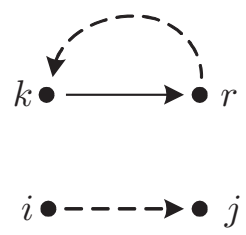

$g$

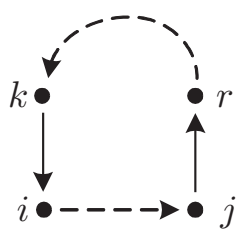

$g^{\prime \prime}$

Figure 2.6: Described situation in networks $g$ and $g^{\prime \prime}$. Dashed arcs are directed paths.

All agents receive non-negative payoffs in $g^{\prime}=g+(k, i)$; due to 2.9 agent $r$ can improve in $g^{\prime}$ by replacing link $(k, r)$ with $(j, r)$, thus obtaining $g^{\prime \prime}$. The payoffs of the other agents do not decrease, so all agents receive non-negative payoffs in $g^{\prime \prime}$, and again this is in contradiction with the choice of $g$ as there is one more link in $g^{\prime \prime}$ than in $g$. 


\subsection{Architecture of Nash networks}

In this section we provide a characterization of the architecture of Nash networks, for games with various settings of homogeneity and heterogeneity among link costs and profits. First of all, we require the following definitions. A network $g$ is called strongly connected if each agent in $N$ observes all other agents. A network $g$ is called minimal if for each link $(j, i) \in g, j$ is not observed by $i$ in network $g-(j, i)$.

Bala and Goyal (2000a) study the architecture of Nash networks of games with homogeneous link costs and profits. They show that Nash networks are either empty or minimal strongly connected. Some examples of minimal strongly connected networks are depicted in Figure 2.7 .
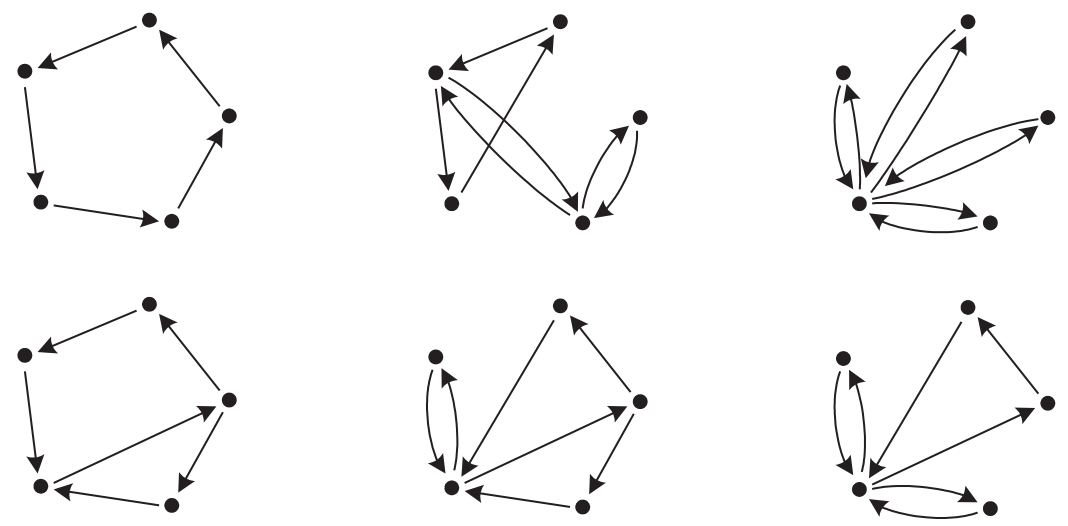

Figure 2.7: Minimal strongly connected networks.

In this section we characterize Nash networks for games with heterogeneous link costs and for games with owner-homogeneous link costs. Here, we will assume that link costs and profits are strictly positive. This assumption, which is also made by Bala and Goyal (2000a) and Galeotti (2006), is needed to obtain insightful results on the architecture of Nash networks. In games with link costs and/or profits equal to 0 , agents often have multiple payoff equivalent actions, which causes a large variety of Nash network architectures. In fact, for games with all link costs and profits equal to 0, a Nash network can have any architecture.

For games with strictly positive link costs, we show in the following lemma that all Nash networks are minimal networks.

Lemma 2.3.1. If $\pi$ is a joint BEGG-1 function with strictly positive link costs, then each Nash network is a minimal network.

Proof. Suppose to the contrary that a Nash network $g$ exists for a game with strictly positive link costs, that is not a minimal network. Then a link $(j, i)$ exists in $g$ and an 
additional directed path from $j$ to $i$. Since link costs are strictly positive, the deletion of link $(j, i)$ is a strictly improving action contradicting that $g$ is a Nash network.

For games with heterogeneous link costs, we know by Example 2.2.9 that Nash networks do not always exist. The Nash networks that do exist for these games are fully characterized as minimal networks:

Theorem 2.3.2. If $\pi$ is a joint BEG-1 function with heterogeneous and strictly positive link costs, then each Nash network is a minimal network. Further, any minimal network is a Nash network for some joint BEGG-1 function with heterogeneous and strictly positive link costs.

Proof. The first part directly follows from Lemma 2.3.1.

Now, let $g$ be a minimal network. Let the profits be $v_{i j}=1$ for all $i, j \in N$. For each link $(j, i)$ in $g$, let $c_{i j}=1$. For each $(j, i) \notin g$, let $c_{i j}=n$.

First we can conclude that any best response of an agent $i$, is a subset of $N_{i}^{d}(g)$, since each link $(j, i)$ where $j \notin N_{i}^{d}(g)$ has cost $c_{i j}=n$, and is therefore not profitable in any situation.

Let $(j, i)$ be a link in $g$. Since $g$ is minimal, $i$ uniquely observes $j$ via $(j, i)$. Since $c_{i j}=v_{i j}=1$, it follows that $j$ can be included in any best response. Therefore we conclude that $N_{i}^{d}(g)$ is a best response of $i$. Hence, $g$ is a Nash network.

Observe that this result holds for both homogeneous and heterogeneous profits.

When link costs are owner-homogeneous, i.e. $c_{i j}=c_{i}$ for all $i, j$, then not all minimal networks are supported as Nash networks. Consider for instance the network with two outgoing links: $g=\{(i, j),(i, k)\}$. This network cannot be Nash for any game with owner-homogeneous link costs, because since $c_{j i}=c_{j k}$, and $v_{j k}>0$, agent $j$ can improve by replacing link $(i, j)$ by $(k, j)$.

To characterize the set of Nash networks for owner-homogeneous link costs, we introduce the following network architecture. Let network $g$ be called semi-proper if for all $(i, j),(i, k) \in g$, with $j \neq k$, link $(i, j)$ is contained in a cycle, and link $(i, k)$ is contained in a cycle.

Observe that all strongly connected networks are semi-proper, including the networks depicted in Figure 2.7. In the following theorem we show that the class of minimal semi-proper networks fully characterizes the set of Nash networks for games with owner-homogeneous links costs. An illustrative example of a minimal semi-proper network is depicted in Figure 2.8

Theorem 2.3.3. If $\pi$ is a joint BEG-1 function with owner-homogeneous and strictly positive link costs, and heterogeneous and strictly positive profits, then each Nash network is minimal and semi-proper. Further, any minimal semi-proper network is a Nash network for some joint BEG-1 function with owner-homogeneous and strictly positive link costs, and heterogeneous and strictly positive profits. 


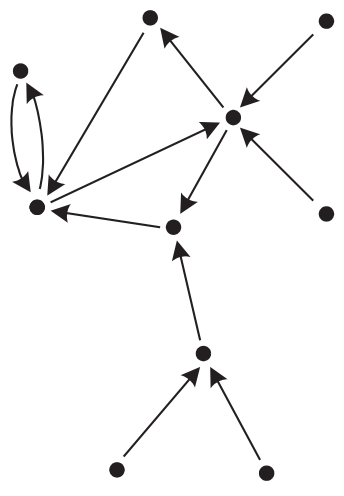

Figure 2.8: A minimal semi-proper network.

Proof. Let $g$ be a Nash network for a game with owner-homogeneous and strictly positive link costs, and strictly positive profits. By Lemma 2.3.1 it follows that $g$ is minimal. Now suppose that $g$ is not semi-proper. Hence two links exist in $g$, say $(i, j)$ and $(i, k)$, such that at least one of them, say $(i, j)$ is not contained in a cycle. It follows that $j \notin N_{k}(g)$. Since link costs are owner-homogeneous and profits are strictly positive, the replacement of $(i, k)$ by $(j, k)$ is strictly improving, implying that $g$ is a not Nash network. Hence we conclude each Nash network is minimal and semi-proper.

Now, let $g$ be a minimal semi-proper network. We prove that a joint B\&G-1 function exists for which $g$ is a Nash network.

First, we require the following definitions. For each $(j, i) \in g$, let $S_{i j}$ be the set of agents that $i$ observes exclusively via $(j, i)$, i.e.

$$
S_{i j}=N_{i}\left(g_{-i j}\right) \backslash N_{i}(g-(j, i)) .
$$

Observe that $j \in S_{i j}$, for otherwise $g$ is not a minimal network. Further, let

$$
S_{i}=\bigcup_{j \in N_{i}^{d}(g)} S_{i j} .
$$

Consider the following B\&G-1 functions. Let link costs be homogeneous: let $c=1$. For each $i$ and $j$, let $v_{i j}=1$ when $j \in S_{i}$ and let $v_{i j}=\frac{1}{n}$ when $j \notin S_{i}$.

We show that for each agent $i \in N$, the agent set $N_{i}^{d}(g)$ is a best response. Let $B$ be a best response of agent $i$, and let the obtained network be $g^{\prime}$.

Consider agent $j \in N_{i}^{d}(g)$. Suppose that $j \notin B$. Then we may assume that $N_{i}\left(g^{\prime}\right) \supseteq$ $S_{i j}$, for otherwise the addition of $(k, i)$, with $k \in S_{i j} \backslash N_{i}\left(g^{\prime}\right)$, is improving since $v_{i k}=c$. Let $j^{\prime}$ be an agent in $B$ by whom $i$ observes an agent $k \in S_{i j}$ in $g^{\prime}$. Since a directed path exists from $k$ to $j^{\prime}$ in $g$ and a directed path from $k$ to $j$ in $g$, by semi-properness, a directed path exists from $j^{\prime}$ to $k$ in $g$ and a directed path from $j$ to $k$ in $g$. Hence, a directed path exists from $j^{\prime}$ to $j$ in $g$ and vice versa. Since link costs are homogeneous, 
the action $B \backslash\left\{j^{\prime}\right\} \cup\{j\}$ is as good as $B$. Hence, without loss of generality, we may assume that $N_{i}^{d}(g) \subseteq B$.

Suppose that $T=B \backslash N_{i}^{d}(g)$ is non-empty. By the fact that link costs are strictly positive, it follows that $T$ only contains newly observed agents i.e. $T \cap N_{i}(g)=\emptyset$. Hence, $T \cap S_{i}=\emptyset$, and therefore it follows that for each $j \in T, v_{i j}=\frac{1}{n}$. Since $c=1>\sum_{j \in T} v_{i j}$, we conclude that $T=\emptyset$, and thus $N_{i}^{d}(g)$ is a best response of $i$ in network $g$.

For games with owner-homogeneous link costs and homogeneous profits, some minimal semi-proper networks are never Nash. Consider for instance the network depicted in Figure 2.8. Let $i$ be the agent who is directly linked with the two leaf agents (i.e. agents without own links) depicted at the bottom. He gains from observing the two leafs. If link costs are owner-homogeneous and profits are homogeneous and strictly positive, then it is also worthwhile to observe the strongly connected subnetwork. Therefore, this network cannot be Nash for games with owner-homogeneous link costs and homogeneous profits.

It can easily be verified that for those games, the architecture of Nash networks is characterized as any minimal semi-proper network $g$ for which the following holds: network $g^{\prime}=g-\left\{(j, i): N_{j}^{d}(g)=\emptyset\right\}$ contains at most one non-empty component, and furthermore, any such component is minimal and strongly connected. Examples of these specific minimal semi-proper networks are depicted in Figure 2.9. The network depicted in Figure 2.9(c), is only Nash if $c_{i}=v$ with $i$ being the agent depicted at the bottom left, because if $c_{i}>v$, then $i$ can better remove his link with the upper agent, and if $c_{i}<v$, then $i$ can better link up with the isolated agent.

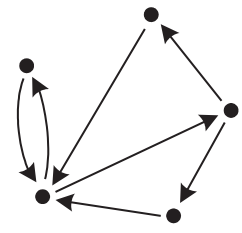

(a)

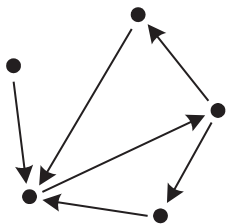

(b)

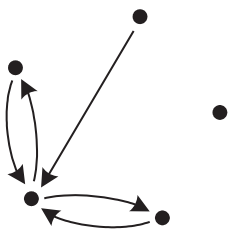

(c)

Figure 2.9: Special cases of minimal semi-proper networks.

\subsection{Strict Nash networks}

Recall that network $g$ is a strict Nash network if $N_{i}^{d}(g)$ is a unique best response for each agent $i$. In this section, we study the existence and architecture of strict Nash networks. 
Bala and Goyal (2000a) prove the existence of strict Nash networks for games with homogeneous link costs and profits. Necessarily, link costs have to be strictly positive for this result, due to the following proposition. Here, we show that strict Nash networks do not exist for any game where all links are for free.

Proposition 2.4.1. Let $N=\{1, \ldots, n\}$ be the set of agents with $n \geq 2$. Let all links be for free, i.e. $c=0$. Then strict Nash networks do not exist.

Proof. Suppose to the contrary that a strict Nash network exists, say $g$. The current action in $g$ for each agent $i$ has to be $N \backslash\{i\}$, because otherwise all supersets of his action are equally good, contradicting that $g$ is a strict Nash network. Hence, we conclude that $g$ is the complete network. But then, each agent $i$ can deviate by playing a non-empty action, which gives him an equal payoff. We conclude that strict Nash networks do not exist.

For games with owner-homogeneous link costs, the following example shows that strict Nash networks may not exist, even if link costs are strictly positive.

Example 2.4.2. Let again $N=\{1, \ldots, n\}$ be the set of agents. Let $\pi$ be a joint B\&G-1 function where $c_{1}=n-1$, and $c_{i}=1$ for all $i \neq 1$. Further, $v_{i j}=1$ for all $i, j \in N$.

For each agent $i \in N \backslash\{1\}$ we have $c_{i}=1<\sum_{j \in N, j \neq i} v_{i j}=n-1$. According to Bala and Goyal (2000a)'s Proposition 3.2, all agents in $N \backslash\{1\}$ form a cycle in each strict Nash network.

Either agent 1 is also contained in this cycle or not. Suppose he is. Then by his one link he receives $n-1$ profits, and the link itself costs $n-1$. Hence, he is indifferent about maintaining this link. Thus, a cycle network cannot be strict Nash.

Now suppose that agent 1 is not contained in the cycle on $N \backslash\{1\}$. Then, by forming a link with one of the other agents, agent 1 receives $n-1$ profits, and pays $n-1$. Therefore, he is indifferent about forming such a link. Hence, again the network cannot be strict Nash. Therefore, we conclude that strict Nash networks do not exist for this game.

The architecture of strict Nash networks is characterized by Bala and Goyal (2000a) and Galeotti (2006). Their results are the following:

* For games with heterogeneous link costs, strict Nash networks are characterized by Galeotti 2006) as minimal networks.

* For games with owner-homogeneous link costs and heterogeneous profits, Galeotti (2006) characterizes strict Nash networks as proper networks, i.e. networks where each agent has at most one outgoing link. An illustrative example of a proper network is depicted in Figure 2.10. Notice that each proper network is minimal. This characterization is confirmed by Example 2.2.4. The network depicted in Figure 2.2 is not strict Nash, because agent $j$ can deviate by forming $(k, j)$ instead 
of $(i, j)$ which gives him the same payoff due to link cost owner-homogeneity. Notice that in the newly obtained network, agent $i$ can deviate by removing link $(k, i)$ which gives him a higher payoff. The cycle network that we now obtain is strict Nash.

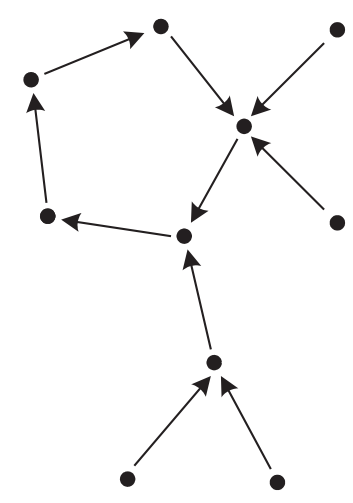

Figure 2.10: A proper network.

* For games with owner-homogeneous link costs and homogeneous profits, strict Nash networks are characterized by Galeotti (2006) as specific proper networks, namely: the empty network, a cycle network, a center-sponsored star (Figure 2.11(a)), a cycle with singletons (Figure 2.11(b)), and a cycle with local center-sponsored stars (Figure 2.11(c)).

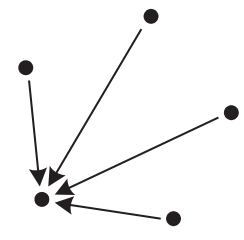

(a)

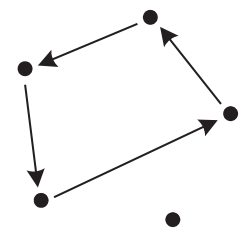

(b)

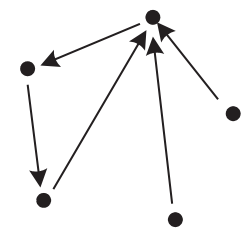

(c)

Figure 2.11: Special cases of proper networks.

* Bala and Goyal (2000a) show that a strict Nash network is either the empty network or a cycle network for games with homogeneous and strictly positive link costs and profits.

Although the characterizations by Bala and Goyal (2000a) and by Galeotti (2006) apply to games with strictly positive link costs and profits, it can be verified as follows that they also apply to games with non-negative link costs and profits. 
* Consider for instance the case where link costs are owner-homogeneous and nonnegative, and where profits are heterogeneous and non-negative. Suppose that there exists a non-proper strict Nash network $g$. Then, an agent $i$ exists who has at least two outgoing links, say $(i, j)$ and $(i, k)$. Since $g$ is strict Nash, agent $j$ strictly prefers $(i, j)$ over $(k, j)$. Since link costs are owner-homogeneous, this implies that $v_{j k}$ is negative; a contradiction. We conclude that each strict Nash network is proper. Further, each proper network is strict Nash for some of those B\&G-1 functions, since each game with strictly positive link costs and profits is also a game with non-negative link costs and profits.

* Now consider the case with heterogeneous and non-negative link costs and profits. Suppose that there exists a non-minimal strict Nash network $g$. Thus, a link $(j, i)$ exists in $g$ and also a directed path from $j$ to $i$. This implies that $c_{i j}$ is negative; a contradiction. We conclude that each strict Nash network is minimal. Like the previous case, the second part is easily established.

Observe that these generalizations are not empty, since strict Nash networks exist when some profits are 0. For instance the empty network is strict Nash when all profits are 0 and all link costs are strictly positive.

\subsection{Negative profits}

In this section we examine games where we drop the assumption that profits should be non-negative. This extension can be very realistic in applications of network formation. Imagine there is a person in your friendship network you do not like. Of course, a friendship with this person is not obligatory. However, some of your friends might have a friendship with this person. Therefore, is it not always good to have as many friends as possible. Another application of network formation where profits may be negative, is the World Wide Web, where you want to avoid certain websites.

In this section we examine games where some profit values are negative. We show by means of a counterexample that the existence of Nash networks is not guaranteed. Further, we provide a full characterization of (strict) Nash networks.

Recall from Lemma 2.2.1 that for games with non-negative profits and costs, any cycle network is Nash whenever the cost of each link is less or equal than the total profit. The following example illustrates that for games with possibly negative profits, cycle networks may not be Nash networks, even if each agent receives a positive payoff.

Example 2.5.1. Let $N=\{1,2, \ldots, n\}$. Let $\pi$ be a joint B\&G-1 function, where the cost of each link is 1 . Let agent 1 be the "dictator". Each other agent $i$ wants to observe him at any cost. However, agent $i$ preferably does not want to observe other agents. Thus for each agent $i \neq 1$, let $v_{i 1}=M$, with $M$ being a very large number, and let $v_{i j}=-1$ for each $j \neq 1$. The dictator himself wants to observe any other agent, i.e. let $v_{1 j}=2$ for all $j$. 


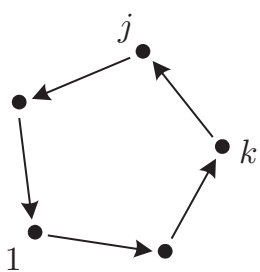

(a)

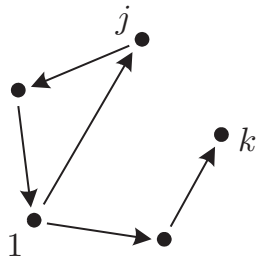

(b)

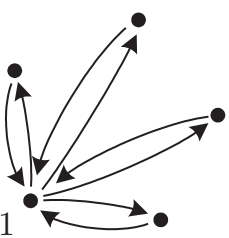

(c)

Figure 2.12: Networks of Example 2.5.1

Consider the cycle network depicted in Figure 2.12(a). This network is not Nash, because agent $j$ (and any other agent except 1 and the agent who observes 1 directly) has an improving action by replacing his link $(k, j)$ with $(1, j)$. See Figure $2.12(\mathrm{~b})$. It can easily be verified that the network depicted in Figure 2.12(c) is the unique Nash network for this game.

Observe that the network in Figure 2.12(a) is at least as good for each agent as the network in Figure 2.12(c), while the former is not Nash and the latter is.

As for the existence of Nash networks, we obtain the following counterexample. For this example, where three agents are involved, Nash networks do not exist.

Example 2.5.2. Let $N=\{1,2,3\}$. Let $\pi$ be a joint B\&G-1 function, where the cost for each link is 1 . Further, let

$$
\begin{array}{ll}
v_{12}=2, & v_{13}=-4, \\
v_{23}=2, & v_{21}=-4, \\
v_{31}=2, & v_{32}=-4 .
\end{array}
$$

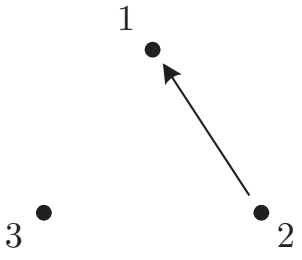

(a)

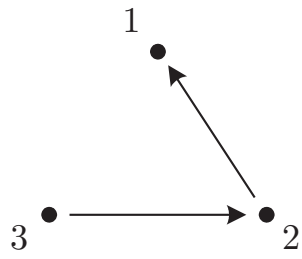

(b)

Figure 2.13: Two networks with only counterclockwise links.

Let the agents 1, 2, and 3 be located as in Figure 2.13. First observe that any Nash network only consists of counterclockwise links (thus $(2,1),(1,3)$, and $(3,2)$ ).

In a network that contains at most one counterclockwise link, an agent exists who can perform a strictly improving addition. For instance, consider the network depicted 
in 2.13 (a). Agent 2 can strictly improve by adding $(3,2)$, obtaining the network in Figure 2.13(b).

Hence any Nash network contains 2 or 3 counterclockwise links. However, in a network with at least 2 counterclockwise links, one of them is not profitable. Consider e.g. the network depicted in Figure 2.13(b). Then $\pi_{1}(g)=-3$, and hence agent 1 can delete $(2,1)$, which is a strictly improving deletion. Hence we conclude that Nash networks do not exist in this example.

In the following theorem we fully characterize Nash networks as minimal networks.

Theorem 2.5.3. If $\pi$ is a joint BEG-1 function with (owner-)homogeneous and strictly positive costs, and heterogeneous (and possibly negative) profits, then each Nash network is a minimal network. Further, any minimal network is a Nash network for some joint BEG-1 function with (owner-)homogeneous and strictly positive costs, and heterogeneous (and possibly negative) profits.

Proof. The first part directly follows from Lemma 2.3.1.

Now, let $g$ be a minimal network. Let the link costs be $c=1$. Further, let $v_{i j}=1$ for each agent $j \in N_{i}(g)$, and $v_{i j}=-n$ for each agent $j \notin N_{i}(g)$.

First we can conclude that any best response of agent $i$ is a subset of $N_{i}(g)$, since each link $(j, i)$ where $j \notin N_{i}(g)$ yields profits $v_{i j}=-n$, and is therefore not profitable in any situation. Let $S \subseteq N_{i}(g)$ be a best response of $i$ in network $g$.

Consider agent $j \in N_{i}^{d}(g)$. Since $v_{i j}=1$, we may assume that $i$ observes $j$ after playing best response $S$. Hence, either $j$ is contained in $S$, or an agent $k$ is contained in $S$ such that a directed path exists from $j$ to $k$ in $g_{-i}$. We have $k \in N_{i}(g)$, for otherwise $v_{i k}=-n$. Hence a directed path from $k$ to $i$ exists in $g$. This path visits $j$, because otherwise $g$ is not minimal (due to link $(j, i)$ ). Hence we conclude that $j$ and $k$ are contained in a cycle that does not contain $i$. Since $c=1, i$ is indifferent about whether he chooses $j$ or $k$. Hence we may assume that $j$ is contained in $S$. Therefore, it follows that $N_{i}^{d}(g) \subseteq S$.

Now consider agent $j \in N_{i}(g) \backslash N_{i}^{d}(g)$. Since $i$ already observes $j$ (indirectly) by playing $N_{i}^{d}(g)$, and since link costs are strictly positive, it follows that $j \notin S$.

We conclude that $N_{i}^{d}(g)$ is a best response of $i$. Therefore, $g$ is a Nash network.

Observe that negative profits have a similar effect on the architecture of Nash networks as heterogeneous link costs (see Theorem 2.3.2). Consider the non-proper network $g=\{(i, j),(i, k)\}$ that is preferred over the proper network $g^{\prime}=\{(k, j),(i, k)\}$ by $j$ whenever $c_{j i}<c_{j k}-v_{j k}$. This implies that either

$* v_{j k} \geq 0$ and $c_{j i}<c_{j k}$, or

$* v_{j k}<0$.

Thus, non-proper networks are supported as Nash networks only when link costs are heterogeneous, or when some profits are negative. We proved that for both cases Nash networks are fully characterized as minimal networks. 
As for strict Nash networks, not all minimal networks are strict Nash for some games with (owner-)homogeneous link costs. Consider for instance a cycle $C$ and an agent $i$ outside $C$ who observes $C$. Then, agent $i$ has multiple payoff equivalent actions: he can replace his link to any agent in $C$. To deal with these situations, we need the following definition: let a network be called cycle-proper if each agent on a cycle has exactly one outgoing link. In the following result, we fully characterize strict Nash networks as minimal cycle-proper networks.

Theorem 2.5.4. If $\pi$ is a joint BEG-1 function with (owner-)homogeneous and nonnegative costs, and heterogeneous (and possibly negative) profits, then each strict Nash network is minimal and cycle-proper. Further, any minimal cycle-proper network is a strict Nash network for some joint BEG-1 function with (owner-)homogeneous and non-negative costs, and heterogeneous (and possibly negative) profits.

Proof. Let $g$ be a strict Nash network for a game with (owner-)heterogeneous link costs. Suppose to the contrary that $g$ is not a minimal network. Then a link $(j, i)$ exists in $g$ and an additional directed path from $j$ to $i$. Since link costs are non-negative, the deletion of link $(j, i)$ is an action of agent $i$ that does not decrease his payoff. This contradicts that $g$ is a strict Nash network.

Now suppose to the contrary that $g$ is not cycle-proper. Then a cycle exists where an agent on it has at least two outgoing links, a link on this cycle $(i, j)$ and another link $(i, k)$. Then, the action $N_{k}^{d}(g) \backslash\{i\} \cup\{j\}$ yields the same payoff for agent $k$ as his current action $N_{k}^{d}(g)$. This implies that $g$ is not strict Nash; a contradiction.

Now, let $g$ be a minimal cycle-proper network. Let the link costs be $c=1$. Further, let $v_{i j}=2$ for each agent $j \in N_{i}(g)$, and $v_{i j}=-n$ for each agent $j \notin N_{i}(g)$.

First we can conclude that any best response of agent $i$ is a subset of $N_{i}(g)$, since each link $(j, i)$ where $j \notin N_{i}(g)$ yields profits $v_{i j}=-n$, and is therefore not profitable in any situation. Let $S \subseteq N_{i}(g)$ be a best response of $i$ in network $g$.

Consider agent $j \in N_{i}^{d}(g)$. Since $g$ is minimal, $i$ uniquely observes $j$ via $(j, i)$. Since $v_{i j}>c$, it follows that agent $i$ observes $j$ after playing $S$. Hence, either $j \in S$ or an agent $k \neq j$ is contained in $S$ such that a directed path exists from $j$ to $k$ in $g_{-i}$. In the latter case, we may assume that $k \in N_{i}(g)$, because otherwise $v_{i k}=-n$. Thus, a directed path from $k$ to $i$ exists in $g$. This path visits $j$, for otherwise $g$ is not a minimal network (due to link $(j, i)$ ). Hence, $j$ and $k$ are contained in a cycle that does not contain $i$. Thus, agent $j$ has two outgoing links: one on the cycle and one to agent $i$. This contradicts the fact that $g$ is cycle-proper. Hence we conclude that such an agent $k$ does not exist. We conclude that $j$ is contained in $S$. Therefore, we have $N_{i}^{d}(g) \subseteq S$.

Now consider agent $j \in N_{i}(g) \backslash N_{i}^{d}(g)$. Since $i$ already observes $j$ (indirectly) by playing $N_{i}^{d}(g)$, and since $c=1$, it follows that $j \notin S$.

Therefore we conclude that $N_{i}^{d}(g)$ is the unique best response of $i$. Hence, $g$ is a strict Nash network. 


\subsection{Overview of the results}

In this section we provide an overview of the results with respect to the architecture and existence of Nash networks in the one-way flow model. In Table 2.1. these results are summarized. Here, all link costs and profits are non-negative, unless mentioned otherwise.

\begin{tabular}{|c|c|c|c|c|c|}
\hline \multirow{2}{*}{$\begin{array}{l}\text { Payoff } \\
\text { Costs }\end{array}$} & \multirow[b]{2}{*}{ Profits } & \multicolumn{2}{|c|}{ Nash networks } & \multicolumn{2}{|c|}{ Strict-Nash networks } \\
\hline & & Existence & Architecture $^{1}$ & Existence & Architecture \\
\hline \multirow[t]{2}{*}{ Homogeneous } & Homogeneous & Yes & $\begin{array}{l}\text { Empty network, } \\
\text { minimal strongly } \\
\text { connected network }\end{array}$ & Yes $^{2}$ & Empty network, cycle \\
\hline & & (BG2000a) & $(\mathrm{BG} 2000 \mathrm{a})$ & (BG2000a) & (BG2000a) \\
\hline \multirow[t]{2}{*}{$\begin{array}{l}\text { Owner- } \\
\text { homogeneous }\end{array}$} & Homogeneous & Yes & $\begin{array}{l}\text { Special case of } \\
\text { minimal semi-proper } \\
\text { network }\end{array}$ & No & $\begin{array}{l}\text { Special case of proper } \\
\text { network }\end{array}$ \\
\hline & & $\begin{array}{l}\text { (Thms } 2.2 .2 \\
\text { and } 2.2 .8\end{array}$ & (Fig. 2.9 & (Ex.2.4.2 & (Gal2006) \\
\hline \multirow[t]{2}{*}{$\begin{array}{l}\text { Owner- } \\
\text { homogeneous }\end{array}$} & Heterogeneous & Yes & $\begin{array}{l}\text { Minimal semi-proper } \\
\text { network }\end{array}$ & No & Proper network \\
\hline & & $\begin{array}{l}\text { (Thms } 2.2 .2 \\
\text { and } 2.2 .8\end{array}$ & (Thm. 2.3 .3 & (Ex.2.4.2 & (Gal2006) \\
\hline \multirow[t]{2}{*}{$\begin{array}{l}\text { (Owner-) } \\
\text { homogeneous }\end{array}$} & $\begin{array}{l}\text { Possibly } \\
\text { negative }\end{array}$ & No & Minimal network & No & $\begin{array}{l}\text { Minimal cycle-proper } \\
\text { network }\end{array}$ \\
\hline & & (Ex.2.5.2 & (Thm. 2.5 .3 & (Ex.2.4.2 & (Thm. 2.5.4 \\
\hline \multirow[t]{2}{*}{ Heterogeneous } & Homogeneous & No & Minimal network & No & Minimal network \\
\hline & & (Ex.2.2.9 & (Thm. 2.3.2 & (Ex.2.4.2 & (Gal2006) \\
\hline \multirow[t]{2}{*}{ Heterogeneous } & Heterogeneous & No & Minimal network & No & Minimal network \\
\hline & & (Ex.2.2.9 & (Thm. 2.3.2 & (Ex.2.4.2 & (Gal2006) \\
\hline
\end{tabular}

Table 2.1: Overview of the results.

\footnotetext{
${ }^{1}$ For the architecture of Nash networks, we assume that link costs and profits are unequal to 0 , for otherwise a Nash network can have any architecture.

${ }^{2}$ Strict Nash networks only exist when $c>0$.
} 


\section{Chapter 3}

\section{Best response problem}

In the model of network formation that we studied in the previous chapter, an action of agent $i$ is defined as a set of agents $S \subseteq N \backslash\{i\}$ to whom he forms the links $\{(j, i): j \in S\}$. Given a specific network, where the actions of all other agents are fixed, each action yields a certain payoff. An action is called a best response if it yields a maximal payoff for him.

The question that we would like to answer in this chapter is how difficult it is to find such a best response. We will focus on payoff functions of the one-way flow model. Since an action is defined as $S \subseteq N \backslash\{i\}$, the number of possible actions is exponential in the number of agents: $2^{n-1}$, where $n=|N|$. It turns out that the problem of finding a best response is indeed very difficult to solve, even if link costs are homogeneous. Formally, we prove that this problem is NP-hard, by reduction from the Minimum Set Cover problem.

Further, we study algorithms for finding a best response. First we introduce a brute-force algorithm that checks each of the $2^{n-1}$ actions, and selects a best one. Then we propose an algorithm that reduces this number by acquiring knowledge from the network architecture and the payoff function. Although worst case networks exist for which this algorithm performs as bad as brute-force, on the average, the number of actions that it checks is strongly reduced. For this purpose, we run experiments for various values of network density and various numbers of agents.

Finally, we relate the problem of finding a best response with the two-way flow model. The only difference with the one-way flow model, is that profits flow in both directions of the links. We propose an algorithm that finds a best response in polynomial time.

Fabrikant et al. (2003) show that finding a best response in the two-way flow model with decay (here the profits decrease linearly with the distances of the connection paths) is NP-hard. Baron et al. (2008) also study the computational complexity of finding best responses in network formation games, but they consider a different class of payoff functions: agent $i$ receives a profit from agent $j$ if both link $(j, i)$ and $(i, j)$ 
exist, and the costs for agent $i$ are quadratic in the number of links he formed.

This chapter is organized as follows. First we introduce the Best Response Problem (BRP). Then, in Section 3.2, we prove that BRP is NP-hard. In Section 3.3 we propose algorithm ARRAC that solves BRP. We study the time- and space complexity of ARRAC and compare this to a brute-force algorithm in Section 3.4. Then, we study the average case performance of ARRAC by experiments in Section 3.5. Finally, in Section 3.6 we study the best response problem for the two-way flow model. We provide a polynomialtime algorithm for this problem.

This chapter is partly based on Derks et al. (2008b).

\subsection{Problem formulation}

In this chapter, we will use the notations that are introduced in Section 2.1.

Recall that we define an action of agent $i$ to network $g$ as a set of agents, denoted as $S \subseteq N \backslash\{i\}$. The obtained network when $i$ plays action $S$ in $g$ is denoted by

$$
g^{\prime}=g_{-i}+\{(j, i): j \in S\},
$$

and his payoff by the B\&G-1 function

$$
\pi_{i}\left(g^{\prime}\right)=\sum_{j \in N_{i}\left(g^{\prime}\right)} v_{i j}-\sum_{j \in S} c_{i j} .
$$

We identify a Best Response Problem (BRP for short) by a tuple $(N, i, c, v, g)$, where $N$ is a set of agents, $i \in N$ is an agent who searches for a best response, $c$ and $v$ are the costs and profits that specify a B\&G-1 function w.r.t. agent $i$, and $g$ is a network on the agents in $N$.

When agent $i$ is searching for a best response in network $g$, his current incoming and outgoing links in $g$ do not have any influence. The latter can be seen as follows. Suppose that $\pi_{i}\left(g^{\prime}\right) \neq \pi_{i}\left(g^{\prime}-(i, j)\right)$ for any obtained network $g^{\prime}$ and any outgoing link $(i, j) \in g^{\prime}$. Since $i$ does not have to pay for this link, he must receive certain profits that flow along this link. However, since these profits flow from him to $j$, he already receives these profits in $g^{\prime}$ without using link $(i, j)$; a contradiction. Therefore, we may assume that agent $i$ does not have incoming or outgoing links in network $g$, which is the network in which agent $i$ searches for a best response.

\subsection{Complexity class}

In this section we study the complexity class of BRP. For this purpose, we require the following definitions from computational complexity theory.

First of all, a problem is called a decision problem if the solution is "yes" or "no". An optimization problem is the problem of finding a best solution from all feasible solutions. Observe that BRP is an optimization problem. 
A decision problem is assigned to the NP (nondeterministic polynomial time) class if the "yes" answer is verifiable in polynomial time. A decision problem is assigned to the $\mathrm{P}$ (polynomial time) class if there exists at least one algorithm to solve that problem in polynomial time, that is, the number of steps of the algorithm is bounded by a polynomial in the length of the input. Clearly, it follows that $\mathrm{P} \subseteq \mathrm{NP}$. However, it is not known whether $\mathrm{P}=\mathrm{NP}$. It is widely believed that this is not the case. A problem (optimization or decision problem) is NP-hard if a polynomial-time algorithm for solving it implies that for any problem in NP, there exists a polynomial-time algorithm for solving it.

In the next theorem, we prove that BRP is NP-hard.

Theorem 3.2.1. BRP is NP-hard, even when the link costs are homogeneous $\left(c_{i j}=c\right.$ for all $i, j \in N)$.

Proof. We prove this by reduction from the Minimum Set Cover problem (MSC), which is a well-known NP-hard problem (see Karp (1972)). In other words, we prove that any instance of MSC can be transformed (in polynomial time) into an instance of BRP.

Let $\mathcal{K}=\left\{K_{1}, K_{2}, \ldots, K_{k}\right\}$ be a collection of $k$ non-empty subsets of a finite set $X=\{1,2, \ldots, x\}$ such that $X=\bigcup_{j=1}^{k} K_{j}$. MSC is the problem of finding a subset $\mathcal{K}^{\prime} \subseteq \mathcal{K}$ of minimum cardinality such that every element in $X$ belongs to at least one member of $\mathcal{K}^{\prime}$. Notice that such a set cover $\mathcal{K}^{\prime}$ exists.

Next we show how to reduce MSC to BRP. Let $(N, y, c, v, g)$ be a BRP instance, where $N=\left\{1, \ldots, x, K_{1}, K_{2}, \ldots, K_{k}, w, y\right\}$, where the link costs are homogeneous, i.e. $c=1$, where the profits of agent $y$ have the following values:

$$
v_{y i}= \begin{cases}1 & \text { if } i \in X \\ 1-\frac{1}{2 k} & \text { if } i \in \mathcal{K} \\ 0 & \text { if } i \in\{w, y\}\end{cases}
$$

and where $g \in \mathcal{G}$ is a network that is built up as follows: for each agent $i \in X$ we create a link $(i, w)$ and for each agent $i \in K_{j}$ we create a link $\left(i, K_{j}\right)$. In Figure 3.1 an example of such a network is shown.

We show that finding a best response $S$ for the BRP instance $(N, y, c, v, g)$ solves the problem of finding a minimum subset of $\mathcal{K}$ that covers $X$.

Observe that we may restrict to $S \subseteq\left\{K_{1}, \ldots, K_{k}, w\right\}$, because every $i \in X$ is an element of some $K_{j}$, and therefore agent $y$ would receive at least as much payoff from replacing $i$ by $K_{j}$. Further, if $w \in S$, then $S=\{w\}$, since the cost of any additional link exceeds the extra profits. Hence, either $S=\{w\}$ or $S \subseteq \mathcal{K}$. Observe that the action $\{w\}$ yields the payoff $x-1$ for agent $y$.

Let $\mathcal{K}^{\prime} \subseteq \mathcal{K}$ and let $T$ be an action defined as $T=\mathcal{K}^{\prime}$. Then $T$ yields the following payoff for agent $y$ :

$$
\pi_{y}\left(g_{-y}+\{(i, y): i \in T\}\right)=k^{\prime}\left(1-\frac{1}{2 k}\right)+t-k^{\prime}=-\frac{k^{\prime}}{2 k}+t
$$




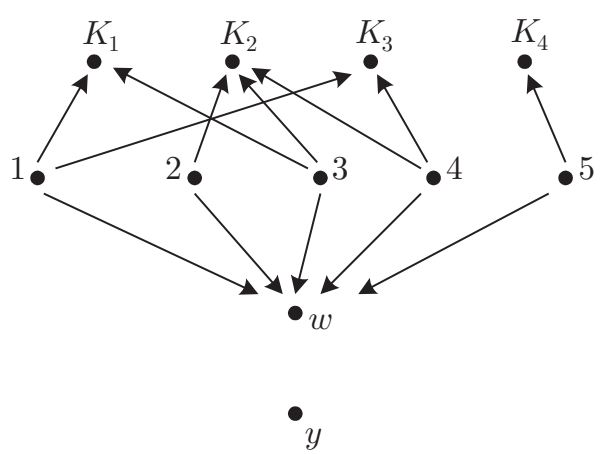

Figure 3.1: Network $g$.

where $k^{\prime}=\left|\mathcal{K}^{\prime}\right|$ and $t$ is the number of members of $X$ that are covered by $\mathcal{K}^{\prime}$.

If $\mathcal{K}^{\prime}$ does not cover $X$, then $t \leq x-1$, and hence $-\frac{k^{\prime}}{2 k}+t \leq-\frac{k^{\prime}}{2 k}+x-1<x-1$. In other words, the action $T$ yields a payoff which is strictly less than the payoff $x-1$ which corresponds to the action $\{w\}$. We conclude that if $\mathcal{K}^{\prime}$ does not cover $X$, then the corresponding action $T$ is not a best response.

If $\mathcal{K}^{\prime} \subseteq \mathcal{K}$ covers $X$, then $t=x$ and hence $-\frac{k^{\prime}}{2 k}+t=-\frac{k^{\prime}}{2 k}+x>x-1$. Thus, the action $T$ yields a strictly higher payoff than the payoff $x-1$ which corresponds to the action $\{w\}$. So every action that is a set cover yields a strictly higher payoff than the payoff from the action $\{w\}$. Of all actions that are set covers, the ones with the lowest cardinality are best responses, because the payoff $-\frac{k^{\prime}}{2 k}+x$ is maximal if $k^{\prime}$ is minimal. We therefore conclude that each best response of agent $y$ with respect to network $g$ is defined as $S=\mathcal{K}^{\prime}$ where $\mathcal{K}^{\prime}$ is a minimum set cover.

Since the transformation from any MSC instance to a BRP instance can be done in polynomial time and since MSC is NP-hard (see Karp (1972)), it follows that BRP is also NP-hard.

Observe that BRP can be interpreted as the problem of maximizing a set function. This can be seen by the fact that agent $i$ chooses a subset from $N \backslash\{i\}$, given a fixed network $g_{-i}$. Let $f: 2^{N \backslash\{i\}} \rightarrow \mathbb{R}$ be a payoff set function defined as

$$
f(S)=\pi_{i}\left(g_{-i}+\{(j, i): j \in S\}\right)
$$

for each $S \subseteq N \backslash\{i\}$, where network $g_{-i}$ and agent $i$ are fixed.

A set function $f$ is called submodular if

$$
f(S)+f(T) \geq f(S \cup T)+f(S \cap T) \text { for all } S, T \subseteq N \backslash\{i\},
$$

supermodular if the left-hand side is less than or equal to the right-hand side, and modular if they are equal. 
For maximizing supermodular set functions in general, which is equivalent to minimizing submodular set functions, Grötschel et al. (1981) proposed a polynomial-time algorithm. Alternative algorithms that are more efficient in practice are proposed independently by Schrijver (2000) and Iwata et al. (2001).

Garey and Johnson (1979) show that the problem of maximizing submodular set functions is NP-hard, due to the fact that it is a general case of the max-cut problem. The problem of maximizing submodular set functions has also been studied by Nemhauser et al. (1978), Lovasz (1983), and Lee et al. (1996), among others.

In the following theorem, we show that the payoff set function $(3.3)$ is submodular.

Theorem 3.2.2. Let $\pi_{i}$ be a BEGG-1 function for agent $i \in N$. Then the corresponding payoff set function $f$, as defined by (3.3), is submodular.

Proof. By definition of B\&G-1 functions in (3.2), we may split $f$ into a profit and a cost part. Therefore we rewrite the profit and the cost function as set functions. Let

$$
v(S)=\sum_{j \in S} v_{i j}
$$

be the profit set function and let

$$
c(S)=\sum_{j \in S} c_{i j}
$$

be the cost set function. Further, we define

$$
N(S)=\left\{j: j \in N_{i}\left(g_{-i}+\{(j, i): j \in S\}\right)\right\},
$$

that is, the set of observed agents by playing action $S$. Now, set function $f$ can be rewritten as

$$
f(S)=v(N(S))-c(S) .
$$

Obviously, the cost set function $c$, defined by (3.5), is modular. To check whether $v$ is modular, submodular, or supermodular with respect to $S$ we have to check the sign of the following expression

$$
v(N(S))+v(N(T))-v(N(S \cup T))-v(N(S \cap T)) .
$$

By $(3.4), v$ is modular with respect to $N(S)$, i.e.

$$
v(N(S))+v(N(T))=v(N(S) \cup N(T))+v(N(S) \cap N(T)) .
$$

By definition (3.6), we conclude that $N(S) \cup N(T)=N(S \cup T)$. Therefore, equation (3.9) can be rewritten as

$$
v(N(S))+v(N(T))=v(N(S \cup T))+v(N(S) \cap N(T)) .
$$


Hence, expression 3.8 can be rewritten as

$$
v(N(S) \cap N(T))-v(N(S \cap T)) .
$$

Since $N(S \cap T) \subseteq N(S)$, and $N(S \cap T) \subseteq N(T)$, it follows that

$$
N(S \cap T) \subseteq N(S) \cap N(T) .
$$

Hence, the sign of expression (3.11) is non-negative. Thus we conclude that profit set function $v$ is submodular. Notice that for disjoint $S$ and $T$ the set $N(S) \cap N(T)$ may be non-empty, while the set $N(S \cap T)$ is empty. In that case, the sign of (3.11) is strictly positive. Therefore, we cannot say that $v$ is not modular in general.

Since $c$ is modular, and $v$ is submodular, the payoff set function $f$ is submodular.

Thus, BRP is a special case of maximizing a submodular set function. By Theorem 3.2.1 we know that even for this case, maximizing a submodular set function is NP-hard. This implies that we have independently proved the result by Garey and Johnson (1979) that maximizing submodular functions in general is NP-hard.

\subsection{Algorithm ARRAC}

In this section we study algorithms that solve BRP, i.e. that find a best response for any instance of BRP. First we introduce the BRUTE-FORCE algorithm, that simply checks all possible actions. Then we present the so-called ARRAC algorithm which enhances the BRUTE-FORCE algorithm in several ways. After this section, we study the complexity of ARRAC in comparison to BRUTE-FORCE, and examine its average case performance.

The BRUTE-FORCE algorithm is straightforward:

Algorithm 3.3.1. BRUTE-FORCE

Input Best response problem $(N, i, c, v, g)$.

Output A best response $B$.

Step Loop through all $2^{n-1}$ actions, and find a best one.

\section{End}

Thus, BRUTE-FORCE checks $2^{n-1}$ possible actions, irrespective of the network.

For unknown payoff functions, BRUTE-FORCE is optimal. However, since we restrict ourselves to B\&G-1 functions (defined by (3.2)), we are able to acquire knowledge from the network architecture. With this knowledge we are able to reduce the number of actions that are candidates for being best responses (and therefore have to be checked). We provide three reduction techniques, and then we propose algorithm ARRAC which is based on them. 
We need the following definitions. First of all, let a set of agents $S \subseteq N$ be called connected in network $g$, if for any two agents $i, j \in S$, an undirected path exists between $i$ and $j$, and this does not hold for any superset $S^{\prime} \supset S$. Notice that an isolated agent is also identified as a connected set. A set of agents $S \subseteq N$, where $|S| \geq 2$, is called a group in network $g$, if for any two agents $i, j \in S$, a directed path exists from $i$ to $j$ and vice versa, and this does not hold for any superset $S^{\prime} \supset S$.

The three reduction techniques are the following.

Connected set approach. Our first reduction technique is founded on the observation that $\mathrm{B} \& \mathrm{G}-1$ functions treat the connected sets in a network separately. More specifically, a B\&G-1 function w.r.t. the obtained network $g^{\prime}$ can be rewritten as

$$
\pi_{i}\left(g^{\prime}\right)=\sum_{\text {connected set } S \text { in } g}\left(\sum_{j \in N_{i}\left(g^{\prime}\right) \cap S} v_{i j}-\sum_{j \in N_{i}^{d}\left(g^{\prime}\right) \cap S} c_{i j}\right) .
$$

The first reduction technique is that per connected set $S$ in $g$, a best response is determined. The union of all these best responses form a best response to network g. This connected set approach strongly reduces the number of actions that have to be checked, especially in sparse networks. For a network that consists of 2 connected sets of the same size, the number of checked actions is already reduced from $2^{n-1}$ to $2\left(2^{\frac{n-1}{2}}\right)=2^{\frac{n+1}{2}}=\sqrt{2^{n+1}}$.

Replacement of groups by representatives. The second technique is based on the following observation. Whenever a group exists in $g$, a connection with one agent in it is sufficient to receive all profits obtained by this group. Thus, for agent $j$ in this group for which $c_{i j}$ is minimal, forming the link $(j, i)$ is sufficient to obtain a maximal payoff from this group. Let this agent $j$ be called the representative of his group (in case there are multiple of them, just pick one arbitrarily).

When agent $i$ plays a best response to a network that contains groups, he only has to consider its representatives. By this observation, the number of actions that agent $i$ has to check in order to find a best response is again reduced (at least, whenever there are groups). How much it is reduced, depends on the network architecture. For instance, when $N \backslash\{i\}$ is a group, $i$ has to check only 2 actions: to form a link with its representative, or to form no links at all.

This brings us to the following reduction technique. A network $g$ is transformed into an acyclic network $g^{\prime}$ as follows. Each group $S$ is replaced by its representative $j$ : each link to (from) an agent in $S$ is replaced by a link to (from) agent $j$. All other group members are left out of consideration. The profit with respect to $j$ is defined as $v_{i j}^{\prime}=\sum_{k \in S} v_{i k}$, while the profits with respect to the other agents do not change. It can easily be verified that a best response to $g^{\prime}$ with modified profits $v^{\prime}$ is also a best response to $g$ with the original profits $v$. 
Selection of rootagents. The third and last technique to obtain valuable information from the network architecture is only applicable when the payoff function satisfies the following property.

Property DE A payoff function $\pi_{i}$ satisfies DE (short for downstream efficiency) if $\pi_{i}(g+(k, i)) \leq \pi_{i}(g+(j, i))$ for any network $g$ where links $(j, i)$ and $(k, i)$ are not contained, and where a directed path from $k$ to $j$ exists.

This property came already into view in Section 2.2.1. 2.5). It is only satisfied by B\&G-1 functions with owner-homogeneous link costs. The intuition behind this, is the following. Whenever a directed path exists from $k$ to $j$, agent $i$ receives at least as much profits via link $(j, i)$ as via link $(k, i)$. Due to owner-homogeneous link costs, i.e. $c_{i j}=c_{i k}=c_{i}$, forming link $(j, i)$ is at least as good as forming link $(k, i)$.

Let an agent be called a rootagent if he does not have outgoing links. The third reduction technique is that, given an acyclic network (i.e. without groups), only actions are checked that only contain rootagents.

So far, we provided three techniques that can be used to reduce the number of actions that have to be checked in order to find a best response. Observe that these techniques only reduce the number of actions by looking at the architecture of the network, and not at the specific costs and profits.

Now we describe an algorithm that incorporates these three reduction techniques. This algorithm will be called ARRAC, which is an abbreviation for Actionspace Reduction to Rootagents in Acyclic Connected sets. We will describe this algorithm with the help of an example. In the network depicted in Figure 3.2, agent $i$ has to find a best response.

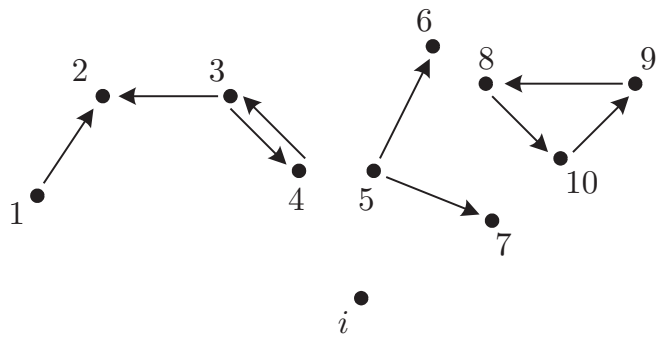

Figure 3.2: Example of network $g$.

\section{Algorithm 3.3.2. ARRAC}

Input Best response problem $(N, i, c, v, g)$.

Output A best response $B$.

Step 1 Find all groups in $g$. In Figure 3.2 there are two groups: $\{3,4\}$ and $\{8,9,10\}$. For this step Tarjan's algorithm (Tarjan (1972)) can be used. 
Step 2 Create a new best response problem $\left(N^{\prime}, i, c, v^{\prime}, g^{\prime}\right)$ as follows.

Put the following agents in set $N^{\prime}$ :

* each agent in $N$ who is not a member of a group, and

* a representative $s$ for each group $S$, such that $c_{i s}=\min _{k \in S} c_{i k}$.

For each $j \in N^{\prime}$, define $v_{i j}^{\prime}$ as:

$$
v_{i j}^{\prime}= \begin{cases}\sum_{k \in S} v_{i k} & \text { if } j \text { represents group } S \\ v_{i j} & \text { otherwise. }\end{cases}
$$

Construct network $g^{\prime}$ by replacing each begin- or endpoint in $g$, that is adjacent to a member of a group, to its representative. Consider for instance the network in Figure 3.3 . Here, the representatives are labeled $s_{1}$ and $s_{2}$. Notice that $g^{\prime}$ is acyclic.

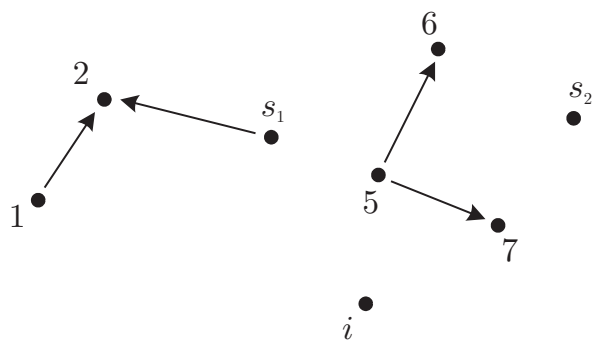

Figure 3.3: Example of network $g^{\prime}$.

Step 3 Find all connected sets in $g^{\prime}$ (except $\{i\}$ ) by using a breadth-first search, and call them $S_{1}, S_{2}, \ldots$ In the example there are three of them: $\left\{1,2, s_{1}\right\},\{5,6,7\}$, and $\left\{s_{2}\right\}$.

Step 4 Whenever link costs are heterogeneous, let $R_{k}=S_{k}$ for each connected set $S_{k}$ in $g^{\prime}$. Otherwise, find all rootagents in $g$ who are member of $S_{k}$, and call this set of rootagents $R_{k}$. In the example, we have $R_{1}=\{2\}, R_{2}=\{6,7\}$, and $R_{3}=\left\{s_{2}\right\}$.

Step 5 Per connected set $S_{k}$ in $g^{\prime}$, calculate the payoff for each subset of $R_{k}$. Thus, $2^{\left|R_{k}\right|}$ actions are considered per connected set $S_{k}$. In our example, there are 2,4 , and 2 of these actions for the first, second, and third connected set respectively. Let $B_{k} \subseteq R_{k}$ be an action with the highest payoff. Let $B=\bigcup_{k} B_{k}$ be the union of these actions over all connected sets. Then, $B$ is a best response to the BRP instance $\left(N^{\prime}, i, c, v^{\prime}, g^{\prime}\right)$, and moreover, it is a best response to the original BRP instance $(N, i, c, v, g)$.

\section{End}


The correctness of ARRAC is easily established by the description of the three reduction techniques prior to this algorithm. Recall that finding rootagents in step 4 requires the payoff function to satisfy $\mathbf{D E}$, which is the case whenever link costs are owner-homogeneous.

\subsection{Time and space complexity of ARRAC}

In this section we will study the complexity of ARRAC. We consider two types of complexity: time and space complexity.

For measuring time and space complexity we will use the 'big $\mathrm{O}$ ' notation, which is written by the symbol $\mathcal{O}$. We say that $f(x)=\mathcal{O}(g(x))$ if there are fixed positive constants $c$ and $k$ such that $0 \leq f(x) \leq c g(x)$ for all $x \geq k$. When we write equality of two $\mathcal{O}$ functions, i.e. $\mathcal{O}(f(x))=\mathcal{O}(g(x))$, then $f(x)=\mathcal{O}(g(x))$ holds, while $g(x)=$ $\mathcal{O}(f(x))$ does not necessarily hold. For example, $\mathcal{O}(x)=\mathcal{O}\left(x^{2}\right)$ whereas $\mathcal{O}\left(x^{2}\right) \neq \mathcal{O}(x)$.

The time complexity of ARRAC is the number of arithmetic operations that it takes to solve a BRP instance using ARRAC, as a function of the number of agents and/or the number of links. Recall that the number of agents in $N$ is $n$. Further, let $e$ be the number of links in $g$.

The space complexity of ARRAC is the amount of memory required to solve a BRP instance using ARRAC. We assume that each data value that ARRAC uses can be expressed with a 16-bit integer, i.e. a natural number between 0 and 65535. Therefore we define the space complexity of ARRAC by the number of stored integers as a function of the number of agents. Observe that the ratio between the amount of storage required to store a 16-bit integer and that of a binary value is 16: a fixed constant. Therefore, the space complexities of storing an integer and a binary value are equal.

We would like to remark that there are four common ways to represent a network by data:

Adjacency matrix: an $n$ by $n$ binary matrix, where an element at row $i$ and column $j$ equals 1 if and only if $(j, i) \in g$.

Incidence matrix: an $n$ by $e$ matrix, where an element at row $i$ and column $\ell$ equals

+1 if link $\ell$ starts at node $i$,

-1 if link $\ell$ ends at node $i$, and

0 otherwise.

Adjacency list: a vertical list of $n$ agents, where for each agent $i$, the agents in $N_{i}^{d}(g)$ are listed horizontally.

Edge list: a list of links.

Notice that the edge list representation corresponds to our mathematical representation of a network. For choosing the most appropriate data representation, we have to 
examine the space complexities of them, and the time complexities of data operations that BRP algorithms, in particular ARRAC, will perform.

We choose the adjacency matrix representation because of the following reasons. The set of agents (i.e. nodes) in a BRP is fixed. Therefore, each elementary network operation that we need, can be run in $\mathcal{O}(1)$ time. Furthermore, it is clear that the space needed to store an adjacency matrix $\left(n^{2}\right.$ bits) will not be a problem.

One part that appears in both algorithms is that different actions are compared to each other in order to find a best one. This means that for each action, the corresponding payoff has to be calculated. In complexity theory, a function evaluation is often considered as one arithmetic step. However, since our payoff function is derived directly from the network and the costs and profits, we take the time and space complexities of calculating a payoff into account. The payoff can be calculated with a breadth-first or depth-first search (in order to determine the set $N_{i}\left(g^{\prime}\right)$, where $g^{\prime}$ is the obtained network).

In the next theorem, we provide the space complexity of ARRAC.

Theorem 3.4.1. Let $(N, i, c, v, g)$ be a best response problem. Then the space complexity of ARRAC is $\mathcal{O}\left(n^{2}\right)$, where $n$ is the number of agents in $N$.

Proof. For storing BRP instances (ARRAC stores two of them), $\mathcal{O}\left(n^{2}\right)$ is needed. Further, space is needed for several depth- and breadth-first searches (in steps 1 , 3, and $5)$. It is known that the space complexities for these searches are $\mathcal{O}(n)$ and $\mathcal{O}(n+e)$ respectively. Therefore, the space complexity of ARRAC is $\mathcal{O}\left(n^{2}\right)$.

Clearly, this space complexity will not be a problem in practice. In the following results we provide the time complexities of BRUTE-FORCE and ARRAC.

Theorem 3.4.2. The time complexity of BRUTE-FORCE is $\mathcal{O}\left((n+e) 2^{n}\right)$, where $n$ is the number of agents in $N$, and $e$ is the number of links in $g$.

Proof. In BRUTE-FORCE, the payoff is calculated for each of the possible $2^{n-1}$ actions. Each of these calculations requires a breadth-first search, which has time complexity $\mathcal{O}(n+e)$. Hence, the time complexity of BRUTE-FORCE is $\mathcal{O}\left((n+e) 2^{n}\right)$.

Theorem 3.4.3. The time complexity of ARRAC is $\mathcal{O}\left((n+e) 2^{n}\right)$, where $n$ is the number of agents in $N$, and $e$ is the number of links in $g$.

Proof. It can easily be verified that the time complexities of ARRAC in steps 1 to 4 are at most $\mathcal{O}(n+e)$, which is the known time complexity of both a depth- and a breadth-first search.

The time complexity of step 5 is $\mathcal{O}\left((n+e) 2^{n}\right)$, due to the following worst case example. Consider a BRP where $g=\{(1, j): j \in N \backslash\{1\}\}$. This network, which is depicted in Figure 3.4 , does not contain groups, thus $g^{\prime}=g$. Next, there are $n-2$ rootagents in $g^{\prime}$ (all agents except $i$ and 1). Therefore, the payoff's of $2^{n-2}$ actions 


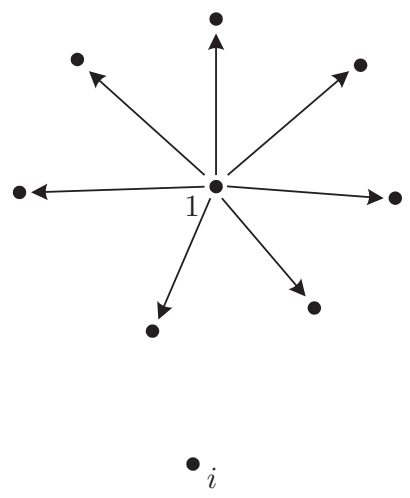

Figure 3.4: Worst case example for algorithm ARRAC.

are calculated by ARRAC. It requires a breadth-first search per calculation (with time complexity $\mathcal{O}(n+e))$. Thus, the time-complexity of step 5 is $\mathcal{O}\left((n+e) 2^{n}\right)$.

Hence, we conclude that the time complexity of the 5 th step is dominant. Therefore the time complexity of ARRAC is also $\mathcal{O}\left((n+e) 2^{n}\right)$.

Observe that both ARRAC's time and space complexity do not depend on whether ARRAC searches for rootagents in step 4.

We showed that the worst case time complexities of ARRAC and BRUTE-FORCE are equal. However, BRUTE-FORCE requires this complexity for any instance of the BRP, whereas ARRAC only requires this complexity for worst case instances, like the network depicted in Figure 3.4. This motivates our focus on the average case analysis of ARRAC in the next section.

\subsection{Average case analysis of ARRAC}

In this section we study the average case performance of ARRAC. From the previous section we know that the number of actions that are checked in step 5 determines the running time of ARRAC. We would like to know how much this number is reduced from $2^{n-1}$ for the average case. Therefore, we will measure the performance of ARRAC by this number.

For 4 to 31 agents, and various density values $d$ between 0 and 1, we generate random networks according to the model proposed by Erdös and Rényi (1960). In this model, each of possible links independently occurs with probability $d$. Since we assumed that agent $i$ does not have incoming or outgoing links, we generate random networks on $n-1$ agents, and then add $i$ as an isolated agent. Thus, the estimated number of links in a random network is $(n-1)(n-2) d$. For each pair $(n, d)$ that we choose, we run ARRAC on 100.000 random networks.

For each of these networks, ARRAC finds a best response. We distinguish two cases: 
one where ARRAC searches for rootagents in step 4, and one where it does not. First we consider the first case, i.e. the case where link costs are (owner-)homogeneous. The average numbers of actions that ARRAC checks in step 5 are shown in Table 3.1. For each of these numbers, the standard error is less than $0.8 \%$.

We also calculated the standard deviation for each pair $(n, d)$. The highest values occurred for intermediate density values. For instance, it is 171 for $n=31$ and $d=0.05$. For low and high density values, the standard deviation is much lower (it is 3.7 for $n=31, d=0.005$ and it is 0.07 for $n=31, d=0.2$ ).

\begin{tabular}{|c|c|c|c|c|c|c|c|c|c|c|}
\hline$n \backslash d$ & 0.005 & 0.01 & 0.025 & 0.05 & 0.075 & 0.1 & 0.125 & 0.15 & 0.175 & 0.2 \\
\hline 4 & 5.94 & 5.88 & 5.71 & 5.43 & 5.16 & 4.91 & 4.67 & 4.45 & 4.24 & 4.03 \\
\hline 5 & 7.88 & 7.77 & 7.43 & 6.89 & 6.38 & 5.92 & 5.49 & 5.10 & 4.75 & 4.41 \\
\hline 6 & 9.80 & 9.61 & 9.05 & 8.20 & 7.41 & 6.71 & 6.08 & 5.52 & 5.01 & 4.56 \\
\hline 7 & 11.70 & 11.41 & 10.59 & 9.36 & 8.28 & 7.32 & 6.50 & 5.77 & 5.11 & 4.53 \\
\hline 8 & 13.59 & 13.19 & 12.05 & 10.40 & 9.00 & 7.82 & 6.80 & 5.90 & 5.12 & 4.43 \\
\hline 9 & 15.45 & 14.93 & 13.44 & 11.35 & 9.67 & 8.26 & 7.06 & 5.95 & 5.03 & 4.23 \\
\hline 10 & 17.30 & 16.62 & 14.76 & 12.23 & 10.28 & 8.70 & 7.29 & 5.97 & 4.87 & 3.97 \\
\hline 11 & 19.11 & 18.28 & 16.00 & 13.04 & 10.92 & 9.11 & 7.45 & 5.91 & 4.63 & 3.68 \\
\hline 12 & 20.92 & 19.91 & 17.20 & 13.87 & 11.64 & 9.63 & 7.56 & 5.73 & 4.31 & 3.36 \\
\hline 13 & 22.72 & 21.51 & 18.34 & 14.73 & 12.44 & 10.12 & 7.58 & 5.45 & 3.99 & 3.07 \\
\hline 14 & 24.48 & 23.07 & 19.43 & 15.72 & 13.49 & 10.59 & 7.44 & 5.12 & 3.65 & 2.81 \\
\hline 15 & 26.24 & 24.61 & 20.50 & 16.88 & 14.59 & 10.89 & 7.21 & 4.72 & 3.33 & 2.61 \\
\hline 16 & 27.99 & 26.10 & 21.57 & 18.36 & 15.90 & 11.11 & 6.86 & 4.32 & 3.05 & 2.46 \\
\hline 17 & 29.69 & 27.58 & 22.60 & 20.05 & 17.26 & 11.15 & 6.43 & 3.96 & 2.82 & 2.33 \\
\hline 18 & 31.39 & 29.03 & 23.71 & 22.40 & 18.54 & 10.92 & 5.92 & 3.59 & 2.63 & 2.24 \\
\hline 19 & 33.07 & 30.43 & 24.85 & 25.09 & 19.74 & 10.65 & 5.48 & 3.31 & 2.49 & 2.17 \\
\hline 20 & 34.73 & 31.79 & 26.12 & 28.60 & 20.76 & 10.05 & 5.00 & 3.08 & 2.37 & 2.12 \\
\hline 21 & 36.38 & 33.17 & 27.58 & 32.67 & 21.35 & 9.54 & 4.57 & 2.85 & 2.28 & 2.09 \\
\hline 22 & 38.01 & 34.46 & 29.24 & 37.14 & 21.88 & 8.84 & 4.18 & 2.68 & 2.21 & 2.06 \\
\hline 23 & 39.64 & 35.81 & 31.14 & 42.88 & 21.77 & 8.18 & 3.82 & 2.54 & 2.15 & 2.04 \\
\hline 24 & 41.24 & 37.09 & 33.73 & 48.55 & 21.57 & 7.53 & 3.53 & 2.43 & 2.11 & 2.03 \\
\hline 25 & 42.80 & 38.36 & 36.85 & 54.75 & 21.18 & 6.89 & 3.26 & 2.33 & 2.09 & 2.02 \\
\hline 26 & 44.38 & 39.60 & 41.20 & 60.60 & 20.28 & 6.32 & 3.06 & 2.26 & 2.06 & 2.01 \\
\hline 27 & 45.92 & 40.84 & 45.93 & 66.22 & 19.12 & 5.76 & 2.89 & 2.21 & 2.05 & 2.01 \\
\hline 28 & 47.46 & 42.09 & 53.11 & 73.05 & 18.19 & 5.28 & 2.72 & 2.16 & 2.04 & 2.01 \\
\hline 29 & 48.97 & 43.28 & 61.16 & 76.77 & 16.91 & 4.90 & 2.60 & 2.12 & 2.02 & 2.00 \\
\hline 30 & 50.47 & 44.48 & 73.39 & 82.16 & 15.74 & 4.46 & 2.49 & 2.10 & 2.02 & 2.00 \\
\hline 31 & 51.94 & 45.70 & 87.87 & 85.36 & 14.68 & 4.14 & 2.40 & 2.07 & 2.01 & 2.00 \\
\hline
\end{tabular}

Table 3.1: The average numbers of actions that ARRAC checks.

Several observations can be made by analyzing this table:

Fix $n$, decrease $d$. As the density goes to 0 , the average number converges to $2(n-1)$. Indeed, in an empty network, there are (besides agent $i$ ) $n-1$ connected sets, i.e. $n-1$ singletons, and for each of them two possible best responses are checked: either the singleton is contained in $B$ or not. We conclude that for low density values, the connected set approach of ARRAC is very effective.

Fix $n$, increase $d$. The number of checked actions converges to 2 as the density increases. This can be explained by the fact that the more the network becomes dense, 
the larger the groups are. For high values of $d$, it is very likely that there will be one group containing all agents. When this is the case, the number of best responses that have to be checked is 2 . Thus, for high density values, the replacement of groups by representatives is very effective.

Fix $d$, increase $n$. For a fixed $d$ and an increasing $n$, the average number of checked actions first increases, then decreases, and finally converges to 2 . This convergence can be explained by looking at the connectivity of the networks. By Erdős and Rényi (1960) we know that the network becomes strongly connected as $n$ increases towards infinity. This can already be seen in Table 3.1 for $d=0.2$. The point at which the number of checked actions starts to decrease depends on $d$. Figure 3.5 illustrates the scope of these points.

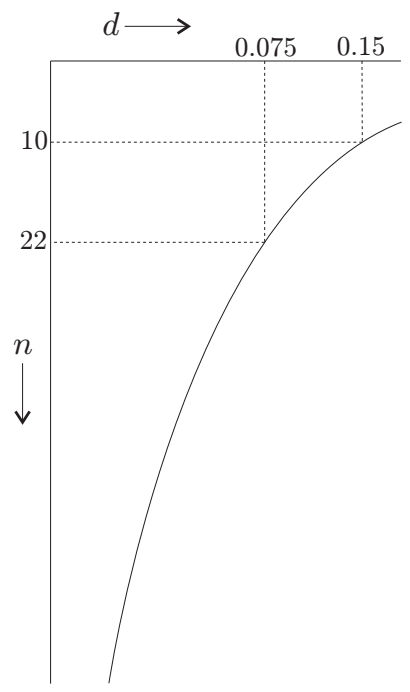

Figure 3.5: Turning points in Table 3.1

For the case where ARRAC does not search for rootagents in step 4, we run the same set of BRP instances. The average numbers of actions that ARRAC checks are shown in Table 3.2. For reference, we also show the numbers of actions that BRUTEFORCE checks: $2^{n-1}$. As can be seen here, ARRAC almost behaves like BRUTE-FORCE for intermediate density values. Observe that also in this case, the number of checked actions will finally converge to 2 as $n$ increases.

By comparing Tables 3.1 and 3.2 , we conclude that finding rootagents in step 4 is an effective reduction technique for intermediate density values.

In Table 3.3 we summarize our observations. Recall that the connectivity of a network depends on both $n$ and $d$. 


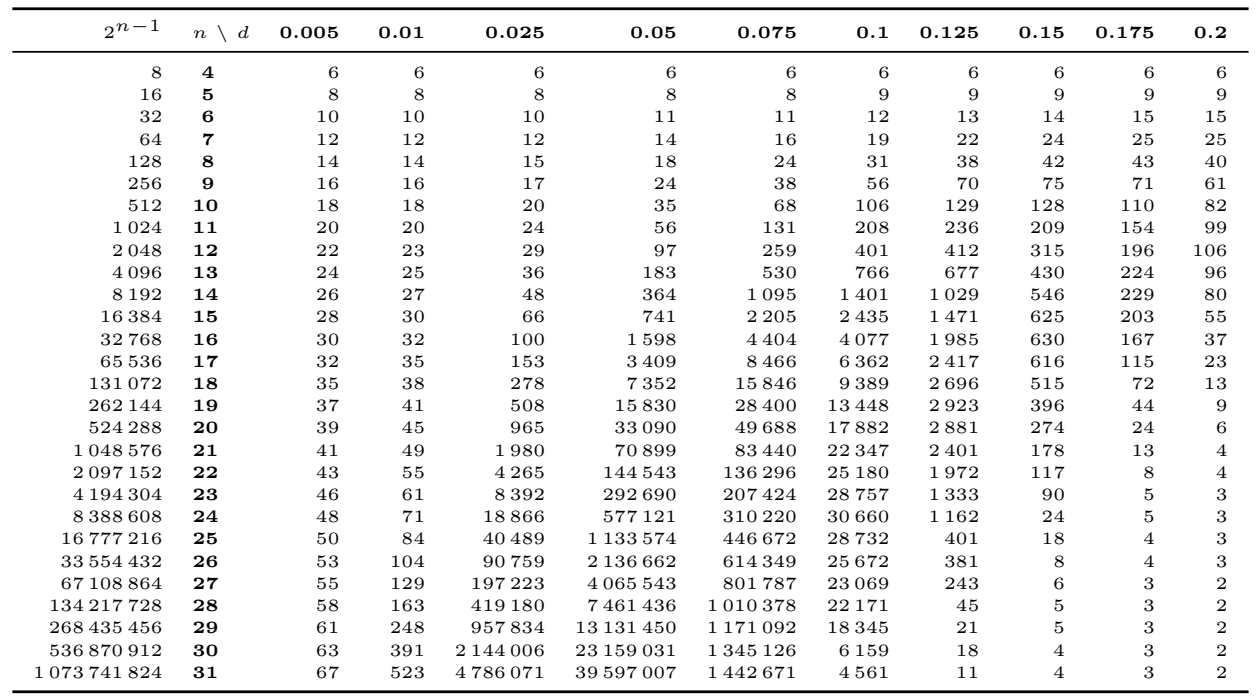

Table 3.2: The average numbers of actions that ARRAC checks without finding rootagents in step 4 , rounded to natural numbers.

\begin{tabular}{ll}
\hline Network connectivity & Dominant reduction technique \\
\hline Low & Connected set approach \\
Medium & Selection of rootagents \\
High & Replacement of groups by representatives \\
\hline
\end{tabular}

Table 3.3: Analysis of ARRAC.

\subsection{Two-way flow case}

Besides the one-way flow model, Bala and Goyal (2000a) also propose the two-way flow model. In Chapter 7, we study the two-way flow model into detail. The only difference with the one-way flow model is that profits flow in both directions of the links. In this section, we propose a polynomial-time algorithm for finding a best response in the two-way flow model, where link costs and profits are heterogeneous.

Let $N_{i}^{u}(g)=\{j \in N$ : an undirected path between $j$ and $i$ exists in $g\}$ be the set of observed agents. The payoff functions of the two-way flow model are given by

$$
\pi_{i}(g)=\sum_{j \in N_{i}^{u}(g)} v_{i j}-\sum_{j \in N_{i}^{d}(g)} c_{i j} \quad \forall i \in N .
$$

Here $v_{i j}$ is the profit that agent $i$ receives from observing $j$ (via an undirected path) and $c_{i j}$ is the cost of link $(j, i)$ for agent $i$. We refer to these payoff functions as B\&G-2 
functions.

We identify a Best Response Problem for the two-way flow model (BRP2 in short) by a tuple $(N, i, c, v, g)$, where $N$ is a set of agents, $i \in N$ is an agent who searches for a best response, $c$ and $v$ are the costs and profits that specify a B\&G-2 function w.r.t. agent $i$, and $g$ is a network on the agents in $N$.

We may assume that $i$ does not have incoming links in $g$. However, we cannot assume that $i$ does not have outgoing links in $g$, because $i$ receives profits via these links.

Consider the following algorithm for solving BRP2, which will be called RCR (short for Replacement of Connected sets by Representatives).

Algorithm 3.6.1. RCR

Input $\operatorname{BRP} 2(N, i, c, v, g)$.

Output A best response $B$.

Step 1 Create a new best response problem $\left(N^{\prime}, i, c, v^{\prime}, g^{\prime}\right)$ as follows.

Put the following agents in set $N^{\prime}$ : a representative $s$ per connected set $S$ in $g$ where $i \notin S$, such that $c_{i s}=\min _{k \in S} c_{i k}$.

For each $j \in N^{\prime}$, define $v_{i j}^{\prime}=\sum_{k \in S} v_{i k}$ where $S$ is the connected set that $j$ represents.

Let $g^{\prime}=\emptyset$.

Step 2 Let $B$ be an action of agent $i$, initiated as the empty set. For each $j \in N^{\prime}$, put $j$ in $B$ if $c_{i j} \leq v_{i j}^{\prime}$. Action $B$ is a best response to the BRP2 instance $\left(N^{\prime}, i, c, v^{\prime}, g^{\prime}\right)$, and moreover, it is a best response to the original BRP2 instance $(N, i, c, v, g)$.

\section{End}

The correctness of this algorithm is easily established.

Observe that this algorithm is similar to some steps in ARRAC. A group in the oneway flow model has the same role as a connected set in the two-way flow model: agent $i$ observes all agents in a connected set by linking up with one of them. Therefore, connected sets are replaced by representatives in RCR. Furthermore, notice that the connected set in which $i$ is active by an outgoing link has no representatives in $N^{\prime}$, because $i$ already receives all profits from this connected set.

The time complexity of step 1 is $\mathcal{O}(n+e)$ because a breadth-first search has to be applied in order to find the connected sets. Further, the time complexity of step 2 is $\mathcal{O}(n)$, since all agents in $N^{\prime}$ have to be checked. Hence, the following theorem is easily established.

Theorem 3.6.2. Algorithm RCR is a polynomial-time algorithm in $n=|N|$. 


\section{Chapter 4}

\section{Local- and global-Nash networks}

In the previous two chapters, we studied a model of unilateral network formation that is introduced by Bala and Goyal (2000a). Each agent forms a set of links. The links of all agents together define a directed network. The links that are formed by agent $i$ are depicted as arcs pointing at $i$. A payoff function assigns a payoff for each agent on base of the formed network.

The payoff functions that Bala and Goyal (2000a) study are the following. Each agent pays a certain cost for each own link, i.e. for each link pointing at him. Further, each agent receives certain profits from observing other agents. Here, two cases are considered. In the first case, agent $i$ observes agent $j$ if a directed path exists from $j$ to $i$, and in the second case if an undirected path exists between them. These two cases are called the one-way flow model (studied in Chapters 2 and 3) and the two-way flow model (studied in Section 3.6 respectively.

Both models are extended by introducing decay (Bala and Goyal (2000a), Kannan et al. (2007), and Feri (2007)), that is, the value of a profit decreases with the length of the connection path counted in number of links. Another extension that is studied in the literature is that links are not perfectly reliable (Bala and Goyal (2000b), Haller and Sarangi (2005), and Haller et al. (2007)).

Observe that all these models (one-way and two-way flow, with and without decay, with perfectly and imperfectly reliable links) only differ from each other in payoff function. In this chapter we prove the existence of Nash networks for a general class of payoff functions. For this purpose, we propose a framework of axiomatic payoff properties that is inspired by the one-way flow model without decay. These properties are intuitive and they are sufficient to guarantee the existence of Nash networks.

Our line of proof and our payoff properties are oriented on local actions, which consist of the following types: an addition, a deletion, and a replacement of a link, and 
a pass. Local actions are easier to analyze than global actions (defined as changing the whole set of own links at once). From a computational point of view, we saw in the previous chapter that the problem of finding a best global response is NP-hard. However, we show that finding a best local response can be done in polynomial time. We use this local approach to obtain global results which are the existence of globalNash networks, and, in the next chapter, the termination of an iterative procedure of local actions at a global-Nash network.

We define a local-Nash network as a network in which no agent can improve by a local action. We prove the existence of local-Nash networks for a framework of payoff properties. Moreover, the networks that we find are proper, i.e. each agent has at most one outgoing link. We show that proper local-Nash networks are also globalNash when the payoff functions satisfy an additional property. We show independence of our properties, and moreover, we show that this framework of properties yields a generalization with respect to the payoff functions in the one-way flow model with owner-homogeneous link costs and heterogeneous profits.

Besides the existence, we study the architecture of local-Nash networks. For the properties that we propose, any network can be local-Nash, for instance when the payoff function equals 0 for each agent and for each network. Therefore, we focus on the architecture of strict local-Nash networks. We show that for payoff functions that satisfy the properties of our framework, strict local-Nash networks are characterized by a larger set of network architectures than proper networks.

The chapter is organized as follows. In Section 4.1 we present the model and the notations that we use throughout. In Section 4.2 we prove that for three payoff properties, each proper local-Nash network is also global-Nash. In Section 4.3 , we prove the existence of proper local-Nash networks for games where the payoff functions satisfy a set of axiomatic properties. As a corollary we obtain the existence of global-Nash networks. In Section 4.4 we study the architecture of (strict) local-Nash networks. We prove independence of our payoff properties in Section 4.5. In Section 4.6 we relate them to payoff functions of the one-way flow model, and provide examples of other payoff functions that satisfy all properties. Finally, in Section 4.7 we provide concluding remarks.

This chapter is mainly based on Derks et al. (2008b).

\subsection{Model and notations}

We will use the same notations that we used throughout Chapters 2 and 3.

Further, let $\operatorname{Car}(g)$, the carrier of $g$, denote the set of so-called active agents in network $g$, being those agents who are begin- or endpoints of a link in $g$.

Recall the following definitions. For a network $g$, we refer to $g^{j}$ as the component of $g$ where agent $j$ is active (see (2.1)). Let $g_{-i}$ be the network obtained from $g$ after removing the links owned by $i$. Notice that an outgoing link of $i$, e.g. $(i, j)$, may still 
exist in $g_{-i}$. Further, we define $g_{-i j}=g_{-i}^{j}+(j, i)$, where $g_{-i}^{j}$ means $\left(g_{-i}\right)^{j}$.

For each agent $i$, let $\pi_{i}: \mathcal{G} \rightarrow \mathbb{R}$ be a payoff function. In this chapter we introduce axiomatic properties for payoff functions in general. These properties are inspired by the one-way flow model.

Recall that an action $S$ of agent $i$ is defined as a subset of agents: $S \subseteq N \backslash\{i\}$. In the sequel we will refer to such an action as a global action. When agent $i$ plays a global action in network $g$, he is able to change his set of links completely. In this chapter, we consider a type of action, where agent $i$ is restricted to change at most one link. Let $S$ be a local action of agent $i$ in network $g$, defined as $S \subseteq N \backslash\{i\}$ where $\left|N_{i}^{d}(g) \backslash S\right| \leq 1$ and $\left|S \backslash N_{i}^{d}(g)\right| \leq 1$. In other words, we consider 4 types of local actions:

(1) passing

(2) adding a new link (pointing at $i$ )

$$
S=N_{i}^{d}(g),
$$

(3) deleting an existing link (pointing at $i$ ) $S=N_{i}^{d}(g) \backslash\{j\}$, and

(4) replacing an existing link by a new link $S=N_{i}^{d}(g) \backslash\{k\} \cup\{j\}$.

The network, after $i$ chooses to link with the agents in $S$ w.r.t. $g$, is described by

$$
g_{-i}+\{(j, i): j \in S\}
$$

A local action $S$ of agent $i$ is called a good local response w.r.t. $\pi_{i}$ and $g$ if

$$
\pi_{i}\left(g_{-i}+\{(j, i): j \in S\}\right) \geq \pi_{i}(g)
$$

A local action $S$ of agent $i$ is called a best local response w.r.t. $\pi_{i}$ and $g$ if

$$
\pi_{i}\left(g_{-i}+\{(j, i): j \in S\}\right) \geq \pi_{i}\left(g_{-i}+\{(j, i): j \in T\}\right)
$$

for all local actions $T$.

A network $g$ is called a local-Nash network if $N_{i}^{d}(g)$ is a best local response for each $i \in N$. A network $g$ is called a strict local-Nash network if $N_{i}^{d}(g)$ is the unique best local response for each $i \in N$.

Analogous definitions apply to the global case. Notice that we used the global case definitions in Chapters 2 and 3.

In Chapter 3, we proved that finding a best global response is NP-hard. In contrast, a best local response is much easier to find. In the following proposition we show that the number of local responses that an agent can choose from, is polynomially bounded by the square of the number of agents. This implies that finding a best local response can be done within polynomial time, because by checking all possible local actions, a best one can be found.

Proposition 4.1.1. The number of possible local actions that an agent can perform, is bounded by $n^{2}$, where $n$ is the number of agents. 
Proof. The number of possible local actions that agent $i$ can perform, depends on the number of neighbors of $i$ in $g$. If we denote this number by $m \leq n-1$, then agent $i$ can do $n-m-1$ additions, $m$ deletions and $(n-m-1) m$ replacements. Hence, the number of possible local actions for agent $i$ equals

$$
\begin{aligned}
n-m-1+m+(n-m-1) m & =(n-1)+(n-m-1) m \\
& \leq(n-1)+(n-m-1)(n-1) \\
& =(n-m)(n-1) \\
& \leq n^{2} .
\end{aligned}
$$

In this chapter we use the local approach in order to prove the existence of localand global-Nash networks. In the next chapter we examine a dynamic game model in which agents play local actions iteratively.

\subsection{Local-Nash and global-Nash networks}

Recall that a network is called proper if the outdegree of each agent is at most one. Further, agent $i$ is called a topagent in network $g$ whenever he observes all agents in his component, i.e. $N_{i}\left(g^{i}\right)=\operatorname{Car}\left(g^{i}\right) \cup\{i\}$. An illustrative proper network, in which the topagents are identified, is depicted in Figure 4.1. Notice that a topagent either is contained in a cycle, or he has no outgoing links. Further, notice that in proper networks also the converse holds.

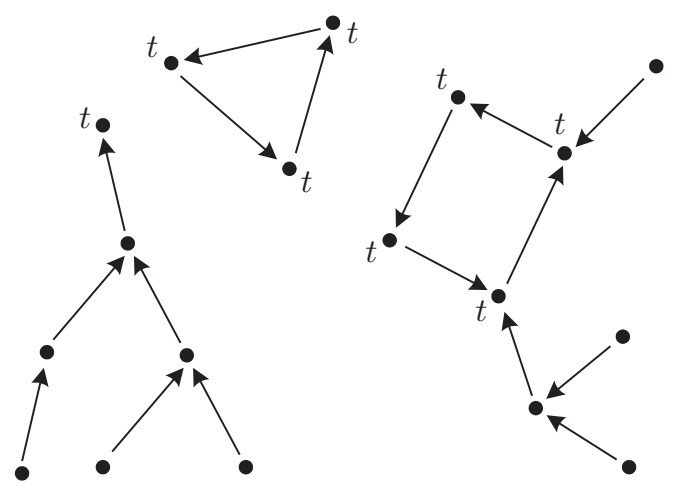

Figure 4.1: A proper network with topagents identified by $t$ 's.

We show that for a specific class of payoff functions, each proper local-Nash network is also global-Nash. This class consists of all payoff functions that have three properties which we will define next: DA (short for disjoint additivity), NA (short for naturality), and DE (short for downstream efficiency). 
Two networks $g$ and $g^{\prime}$ are said to be disjoint with respect to an agent $i$, or $i$-disjoint, if no agent or only agent $i$ is active in both $g$ and $g^{\prime}: \operatorname{Car}(g) \cap \operatorname{Car}\left(g^{\prime}\right) \subseteq\{i\}$.

Property DA We say that a payoff function $\pi_{i}$ is disjoint additive (DA for short), if for each two networks $g$ and $g^{\prime}$, disjoint w.r.t. agent $i$, we have

$$
\pi_{i}\left(g+g^{\prime}\right)=\pi_{i}(g)+\pi_{i}\left(g^{\prime}\right) .
$$

Let a link $(j, i)$ be called profitable in $g$ if:

$$
\pi_{i}(g) \geq \pi_{i}(g-(j, i)) \quad \text { if }(j, i) \in g,
$$

and

$$
\pi_{i}(g+(j, i)) \geq \pi_{i}(g) \text { if }(j, i) \notin g .
$$

Thus, each profitable link that is not present yet is worth adding, and each nonprofitable link that is present is worth deleting. The notion of profitability is very intuitive in network formation.

Another notion which indicates the importance of a link is the following. Let a link $(j, i)$ be called beneficial in $g$ if it is profitable in $g_{-i}^{j}$, i.e.

$$
\pi_{i}\left(g_{-i j}\right) \geq \pi_{i}\left(g_{-i}^{j}\right)
$$

Observe that $(j, i)$ does not have to be contained in $g$. A network is called beneficial if the existing links in that network are beneficial.

Lemma 4.2.1. If network $g$ is proper, and $\pi$ is disjoint additive, then profitability and beneficiality are equivalent notions for all existing links and for some non-existing links:

(i) $\quad \pi_{i}(g)-\pi_{i}(g-(j, i))=\pi_{i}\left(g_{-i j}\right)-\pi_{i}\left(g_{-i}^{j}\right) \quad$ for all $(j, i) \in g$,

(ii) $\quad \pi_{i}(g+(j, i))-\pi_{i}(g)=\pi_{i}\left(g_{-i j}\right)-\pi_{i}\left(g_{-i}^{j}\right) \quad$ for all $(j, i) \notin g$ with $j \notin N_{i}(g)$.

Proof. First, let $(j, i) \in g$. Since $g$ is proper, $g_{-i j}$ and $g-g_{-i j}$ are $i$-disjoint. Therefore, by $\mathbf{D A}$ we have

$$
\pi_{i}(g)=\pi_{i}\left(g_{-i j}\right)+\pi_{i}\left(g-g_{-i j}\right)
$$

Also, $g_{-i}^{j}$ and $g-g_{-i}^{j}$ are $i$-disjoint. Hence by DA we obtain

$$
\pi_{i}(g-(j, i))=\pi_{i}\left(g_{-i}^{j}\right)+\pi_{i}\left(g-g_{-i}^{j}-(j, i)\right) .
$$

With $g-g_{-i j}=g-g_{-i}^{j}-(j, i)$, we obtain (i) from 4.1) and 4.2 .

Now, let $(j, i) \notin g$, with $j \notin N_{i}(g)$. Networks $g_{-i j}$ and $g-g_{-i}^{j}$ are $i$-disjoint, because suppose otherwise: then, an agent $k \neq i$ exists, active in both $g_{-i j}$ and $g-g_{-i}^{j}$. This 
implies that $(k, i) \in g$, because any other link attached to $k$ is inside $g_{-i j}$, and therefore not present in $g-g_{-i}^{j}$. Since $g$ is proper, and $k$ has one outgoing link in it (link $\left.(k, i)\right)$, he cannot have other outgoing links. Therefore, $k$ is a topagent in $g_{-i}^{j}$, and thus observes all agents who are active in $g_{-i}^{j}$, including $j$. This implies that $j \in N_{i}(g)$, which is a contradiction.

Thus, $g_{-i j}$ and $g-g_{-i}^{j}$ are $i$-disjoint, and therefore the networks $g_{-i}^{j}$ and $g-g_{-i}^{j}$ are also $i$-disjoint. By DA we obtain

$$
\pi_{i}\left(g_{-i j}\right)+\pi_{i}\left(g-g_{-i}^{j}\right)=\pi_{i}\left(g_{-i j}+g-g_{-i}^{j}\right)=\pi_{i}(g+(j, i))
$$

and

$$
\pi_{i}\left(g_{-i}^{j}\right)+\pi_{i}\left(g-g_{-i}^{j}\right)=\pi_{i}\left(g_{-i}^{j}+g-g_{-i}^{j}\right)=\pi_{i}(g) .
$$

Hence, (ii) follows from 4.3) and 4.4.

The next payoff property states that connecting to an agent who is already observed is not a strictly improving action.

Property NA We say that $\pi_{i}$ is natural (NA for short) if

$$
\pi_{i}(g+(k, i)) \leq \pi_{i}(g)
$$

whenever $k \in N_{i}(g)$, i.e. there is a directed path from $k$ to $i$ in network $g$.

Thus, in a network where $i$ already observes $k$ via another link, say $(j, i)$, the addition of $(k, i)$ is not an improving action due to NA. The next payoff property can be seen as a "twin" property.

Property DE Payoff function $\pi_{i}$ satisfies DE (short for downstream efficiency) if

$$
\pi_{i}(g+(k, i)) \leq \pi_{i}(g+(j, i))
$$

for any network $g$ where $(j, i) \notin g$ and $(k, i) \notin g$, and where a directed path exists from $k$ to $j$ in $g_{-i}$.

Due to DE, the addition of link $(j, i)$ is at least as good as the addition of $(k, i)$. Observe that the difference between NA and DE is that in the situation where NA is applicable, link $(j, i)$ does exist (on the directed path from $k$ to $i$ ), whereas in the situation where DE is applicable, link $(j, i)$ does not exist.

In the following theorem we show that proper local-Nash networks are also globalNash whenever the payoff functions satisfy the three introduced properties.

Theorem 4.2.2. Let $\pi$ be a joint payoff function that satisfies $\boldsymbol{D A}, \boldsymbol{N A}$, and $\boldsymbol{D E}$. Then each proper local-Nash network is global-Nash. 
Proof. Let $g$ be a proper local-Nash network. Suppose to the contrary that $g$ is not global-Nash, say $i$ can strictly improve in $g$. Let $S=N_{i}^{d}(g)$ be his current action, and let $\tilde{S}$ be a strictly improving action, such that $|\tilde{S} \backslash S|$ is as small as possible and such that among those, $|S \backslash \tilde{S}|$ is as small as possible. Let $\tilde{g}$ be the network obtained after $i$ plays $\tilde{S}$.

First assume $j \in \tilde{S} \backslash S$.

Suppose that $j \in N_{i}(g)$. Then agent $i$ observes $j$ via, say, agent $k \in S$ in $g$, i.e. there is a directed path from $j$ to $k$ in $g_{-i}=\tilde{g}_{-i}$. By DE the action $\tilde{S} \backslash\{j\} \cup\{k\}$ is at least as good as $\tilde{S}$. Now $|(\tilde{S} \backslash\{j\} \cup\{k\}) \backslash S|<|\tilde{S} \backslash S|$, so that we have a contradiction.

Hence $j \notin N_{i}(g)$. Suppose that networks $\tilde{g}-g_{-i j}$ and $g_{-i j}$ are not $i$-disjoint. Since $g_{-i}=\tilde{g}_{-i}$, a link $(k, i)$ exists in $\tilde{g}$ such that $k \in \operatorname{Car}\left(g_{-i}^{j}\right)$. By DE we may assume that $j$ is a topagent in $g_{-i}$, and therefore a directed path exists from $k$ to $j$ in $g_{-i}$. Hence, by $\mathbf{N A}$, it follows that $\tilde{S} \backslash\{k\}$ is at least as good as $\tilde{S}$. Now $|(\tilde{S} \backslash\{k\}) \backslash S|<|\tilde{S} \backslash S|$; a contradiction. Therefore, networks $\tilde{g}-g_{-i j}$ and $g_{-i j}$ are $i$-disjoint. Also, $\tilde{g}-g_{-i j}=\tilde{g}-(j, i)-g_{-i}^{j}$ and $g_{-i}^{j}$ are $i$-disjoint.

By DA we obtain

$$
\begin{array}{ll}
\pi_{i}(\tilde{g}) \quad=\pi_{i}\left(\tilde{g}-g_{-i j}\right) \quad+\pi_{i}\left(g_{-i j}\right), \quad \text { and } \\
\pi_{i}(\tilde{g}-(j, i))=\pi_{i}\left(\tilde{g}-(j, i)-g_{-i}^{j}\right)+\pi_{i}\left(g_{-i}^{j}\right) .
\end{array}
$$

Since $g$ is local-Nash, we have $\pi_{i}(g+(j, i)) \leq \pi_{i}(g)$. By Lemma 4.2.1(ii) and $j \notin N_{i}(g)$, it follows that $\pi_{i}\left(g_{-i j}\right) \leq \pi_{i}\left(g_{-i}^{j}\right)$. Hence by 4.5 and 4.6$)$ we obtain $\pi_{i}(\tilde{g}) \leq \pi_{i}(\tilde{g}-(j, i))$. Hence $\tilde{S} \backslash\{j\}$ is at least as good as $\tilde{S}$, with $(\tilde{S} \backslash\{j\}) \backslash S|<| \tilde{S} \backslash S \mid$. This is a contradiction.

We conclude that $\tilde{S} \subseteq S$.

Now assume $j \in S \backslash \tilde{S}$.

Suppose that $j \in N_{i}(\tilde{g})$, say $(j, k) \in \tilde{g}$. Then, also $(j, k) \in g$. Since $(j, i) \in g$, agent $j$ has two outgoing links, which is a contradiction with the properness of $g$. We conclude that $j \notin N_{i}(\tilde{g})$.

Since $g$ is proper local-Nash, $(j, i)$ is profitable in $g$ and by Lemma 4.2.1(i) also beneficial in $g$. Since $g_{-i}^{j}=\tilde{g}_{-i}^{j}$, link $(j, i)$ is also beneficial in $\tilde{g}$. Further, since $\tilde{g} \subset g$, network $\tilde{g}$ is also proper. By Lemma 4.2.1(ii), $(j, i)$ is also profitable in $\tilde{g}$. Hence $\tilde{S} \cup\{j\}$ is at least as good as $\tilde{S}$, with $|S \backslash(\widetilde{S} \cup\{j\})|<|S \backslash \tilde{S}|$. This is a contradiction.

Hence we conclude that $\tilde{S}=S$, which contradicts $\tilde{S}$ being a strict improvement. Therefore, $g$ is global-Nash.

For more general payoff functions, proper local-Nash networks do not need to be global Nash as can be seen by the following example.

Example 4.2.3. Consider the following payoff function:

$$
\pi_{i}(g)=\left|\left\{j \in N_{i}^{d}(g): j \notin N_{i}(g-(j, i))\right\}\right| .
$$


Consider a network on 4 agents. Let the payoff function $\pi_{1}(g)$ be defined as in (4.7), and let $\pi_{2}(g)=\pi_{3}(g)=\pi_{4}(g)=0$ for all networks $g$. Then, the network depicted in Figure 4.2(a) is a proper local-Nash network. However, it is not global-Nash, because agent 1 can switch to the network depicted in Figure 4.2 (b), which yields payoff 2 instead of 1 . This network is global-Nash.

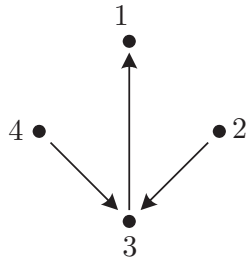

(a)

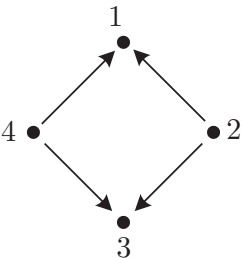

(b)

Figure 4.2: A proper local-Nash network that is not global-Nash (a), and a global-Nash network (b).

Payoff function $\pi_{1}(g)$ satisfies DA, since it can be written as the sum over payoffs w.r.t. 1-disjoint subnetworks. Further, NA is satisfied because the addition of link $(k, 1)$ to a network in which a directed path from $k$ to 1 already exists is not profitable. However, it does not satisfy DE, because agent 1's payoff in the network depicted in Figure 4.2 (b) is strictly higher than his payoff obtained by replacing $(2,1)$ by $(3,1)$. $\diamond$

\subsection{Existence of local-Nash networks}

Next, we identify a class of properties for which we prove the existence of proper localNash networks. For this, we introduce four new properties. These properties only regard beneficiality. Recall that a link $(j, i)$ is beneficial in $g$ whenever $\pi_{i}\left(g_{-i j}\right) \geq$ $\pi_{i}\left(g_{-i}^{j}\right)$. The properties concern how beneficiality of a link is preserved when the network is changed, or how beneficiality of a link depends on the beneficiality of other links.

Property BT Payoff function $\pi_{i}$ satisfies BT (short for beneficial topagent) if the following holds. Let link $(k, i)$ be beneficial in network $g$, and suppose there are topagents in the component $g_{-i}^{k}$. Then there is a topagent $j$ in $g_{-i}^{k}$ such that $\pi_{i}\left(g_{-i j}\right) \geq \pi_{i}\left(g_{-i k}\right)$.

Notice that this property is implied by DE. The following property is also implied by DE.

Property BF Payoff function $\pi_{i}$ satisfies BF (short for beneficial farthest) if the following holds. Let link $(k, i)$ be beneficial in network $g$; let the component $g_{-i}^{k}$ be proper and let agent $i$ be active in $g_{-i}^{k}$ (there is an outgoing link at $i$ in $g_{-i}^{k}$ ). Then also 
link $(j, i)$ is beneficial where $j$ is the agent farthest away from $i$ (counted in number of links) in network $g$.

Notice that since component $g_{-i}^{k}$ is proper and $i$ is active in it, agent $j$ is the unique topagent who is farthest away from $i$ in network $g$. Property BF is also implied by DE. However, it is independent of BT as we will see in Section 4.5. Furthermore, BT and BF do not imply DE. This is illustrated by Example 4.2.3, where the payoff function given by (4.7) does not satisfy DE, whereas it satisfies both BT and BF, since $\pi_{i}\left(g_{-i j}\right)=1$ and $\pi_{i}\left(g_{-i}^{j}\right)=0$ for each network $g$ and each agent $j$.

The following property describes that beneficial links remain beneficial while the network grows:

Property BG Payoff function $\pi_{i}$ satisfies BG (short for beneficial growth) if

$$
\pi_{i}\left((g+(k, r))_{-i j}\right) \geq \pi_{i}\left((g+(k, r))_{-i}^{j}\right)
$$

for any two agents $k, r$, whenever $\pi_{i}\left(g_{-i j}\right) \geq \pi_{i}\left(g_{-i}^{j}\right)$.

Notice that in case we have $r=i$, BG trivially holds, since $(g+(k, r))_{-i j}=g_{-i j}$.

The final property states that beneficiality is preserved when we delete a spoke from the network. Link $(k, r)$ is called a spoke in $g$ if there exists a cycle in $g-(k, r)$ containing both $k$ and $r$.

Property BS Payoff function $\pi_{i}$ satisfies BS (short for beneficial shrink) if

$$
\pi_{i}\left((g-(k, r))_{-i j}\right) \geq \pi_{i}\left((g-(k, r))_{-i}^{j}\right)
$$

whenever $\pi_{i}\left(g_{-i j}\right) \geq \pi_{i}\left(g_{-i}^{j}\right)$ and link $(k, r)$ is a spoke in $g$.

The properties $\mathbf{B F}, \mathbf{B G}$, and $\mathbf{B S}$ are trivially satisfied by payoff functions for which $\pi_{i}(g) \geq \pi_{i}\left(g_{-i}\right)$ for all networks $g$. An example of such a function is $\pi_{i}(g)=\left|N_{i}(g) \backslash\{i\}\right|$ being the number of agents in $g$ observed by $i$. This function also satisfies DA, NA, and BT.

Let us call a payoff function orderly if it satisfies the properties DA, NA, BT, BF, $\mathbf{B G}$, and BS.

Let $\kappa(g)$ be the connection number of network $g$, defined as

$$
\kappa(g)=\sum_{i \in N}\left|N_{i}(g)\right|
$$

Observe that neither the addition of a link nor the deletion of a spoke decreases the connection number.

Theorem 4.3.1. For orderly joint payoff functions any proper beneficial network with maximal connection number is a local-Nash network. 
Proof. Observe that the empty network is proper and beneficial. So, there is a proper beneficial network, say $g$, such that among these networks the connection number $\kappa(g)$ is maximal. We prove that $g$ is local-Nash by deriving a contradiction in the sense that otherwise a proper beneficial network exists with a higher $\kappa$-value than $g$.

So, suppose that $g$ is not local-Nash, say agent $i$ can strictly improve by playing a local action.

Pass: Clearly, this action is not a pass.

Deletion: This local action is neither a deletion because all links of agent $i$ are beneficial, and therefore also profitable by Lemma 4.2.1(i) due to $g$ being proper and $\pi_{i}$ satisfying DA.

Replacement: Suppose the strictly improving local action is a replacement, say link $(k, i)$ is replaced by link $(j, i)$, and let the obtained network be $\tilde{g}=g-(k, i)+(j, i)$. Notice that $k$ is the unique topagent in $g_{-i}^{k}$, since $g$ is proper. If both agents $k$ and $j$ are active in $g_{-i}^{k}$, then, by property $\mathbf{B T}$ it follows that $\pi_{i}\left(g_{-i j}\right) \leq \pi_{i}\left(g_{-i k}\right)$. However, $g-g_{-i k}$ and $\tilde{g}_{-i j}=g_{-i j}$ are $i$-disjoint and their union is $\tilde{g}$, so that by DA we have

$$
\pi_{i}\left(g-g_{-i k}\right)+\pi_{i}\left(g_{-i k}\right)=\pi_{i}(g)<\pi_{i}(\tilde{g})=\pi_{i}\left(g-g_{-i k}\right)+\pi_{i}\left(g_{-i j}\right),
$$

i.e., $\pi_{i}\left(g_{-i j}\right)>\pi_{i}\left(g_{-i k}\right)$; a contradiction.

Therefore, agents $k$ and $j$ are active in different components of $g_{-i}$. The networks $g_{-i k}$ and $g-g_{-i k}+(j, i)$ are $i$-disjoint, with union equal to $g+(j, i)$, and the networks $g_{-i}^{k}$ and $g-g_{-i k}+(j, i)$ are $i$-disjoint, with union $g-(k, i)+(j, i)$. Applying DA twice, we obtain

$$
\begin{aligned}
\pi_{i}(g+(j, i)) & =\pi_{i}\left(g-g_{-i k}+(j, i)\right)+\pi_{i}\left(g_{-i k}\right) \\
& \geq \pi_{i}\left(g-g_{-i k}+(j, i)\right)+\pi_{i}\left(g_{-i}^{k}\right) \\
& =\pi_{i}(g-(k, i)+(j, i)) .
\end{aligned}
$$

The inequality follows from the beneficiality of $(k, i)$ in $g$. We conclude that the addition of $(j, i)$ is at least as good as the replacement of link $(k, i)$ in $g$ by $(j, i)$.

Addition: So, we may assume that the strict improving local action is an addition. Let this addition be $(j, i)$ and let the obtained network be

$$
g^{\prime}=g+(j, i)
$$

If the component $\left(g^{\prime}\right)_{-i}^{j}$, which is equal to $g_{-i}^{j}$, is already linked up with $i$, say $(k, i) \in g$ and $k \in \operatorname{Car}\left(g_{-i}^{j}\right)$, then $k$ is the unique topagent in $g_{-i}^{j}$, due to the properness of $g$. So, there is a directed path from $j$ to $k$ in $g$, and with $(k, i)$ there is a directed path from $j$ to $i$ in $g$, implying $\pi_{i}\left(g^{\prime}\right)=\pi_{i}(g+(j, i)) \leq \pi_{i}(g)$ because of NA. This is a contradiction to the fact that adding $(j, i)$ is strictly improving.

Therefore, the component $\left(g^{\prime}\right)_{-i}^{j}=g_{-i}^{j}$ is not linked up with $i$ in $g$, and by BT we may assume that $j$ is a topagent in $g_{-i}^{j}$. 
Since $g$ is proper, and since $j \notin N_{i}(g)$, by Lemma 4.2.1 and DA, link $(j, i)$ is beneficial in $g^{\prime}$. Also, the other links are beneficial in $g^{\prime}$ due to the beneficiality of $g$ and BG. So, $g^{\prime}$ is a beneficial network. Further, the number $\kappa\left(g^{\prime}\right)$ is higher than $\kappa(g)$, so that $g^{\prime}$ cannot be proper, because by assumption there cannot be proper and beneficial networks with connection number higher than $\kappa(g)$. The only outdegree changed by going from $g$ to $g^{\prime}$ is the one of agent $j$. Therefore, the outdegree of $j$ in $g^{\prime}$ equals 2 , say next to link $(j, i)$, also $(j, k)$ is present in $g^{\prime}$. Since $j$ is a topagent in $g_{-i}^{j}$ there is a directed path from $k$ to $j$ in $g_{-i}^{j}$. Observe that this is also a directed path in $\left(g^{\prime}\right)_{-k}^{j}$.

Extending the directed path from $k$ to $j$ via $(j, i)$ in $\left(g^{\prime}\right)_{-k}^{j}$ in a unique way (since it is a proper network), we arrive at an agent, say $r$, farthest away from $k$ (see Figure 4.3. Since $(j, k)$ is beneficial for $k$, in $g^{\prime}$, also $(r, k)$ is beneficial in $g^{\prime}$ due to $\mathbf{B F}$.

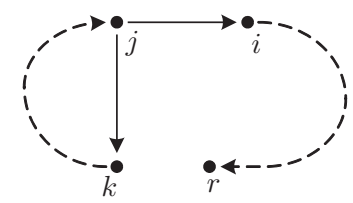

Figure 4.3: The addressed links and directed paths (dashed arcs) of network $g^{\prime}$.

Consider the addition of $(r, k)$ in $g^{\prime}$. From BG and $(r, k)$ being beneficial in $g^{\prime}$, we conclude that $g^{\prime}+(r, k)$ is beneficial. Further, $(j, k)$ is a spoke in this network. After deletion of this spoke, by $\mathbf{B S}$ we again obtain a beneficial network

$$
g^{\prime \prime}=g^{\prime}+(r, k)-(j, k)
$$

with a connection number at least as high as $\kappa\left(g^{\prime}\right)$ and thus higher than $\kappa(g)$. Hence $g^{\prime \prime}$ cannot be proper. This implies that the outdegree of agent $r$ is greater than 1 in $g^{\prime \prime}$. Besides $(r, k)$ we have another link, say $(r, s)$, and $s$ is necessarily located on the unique directed path from $k$ to $r$ in $g^{\prime}$, for otherwise, $s$ would be farther away from $k$ than $r$ is.

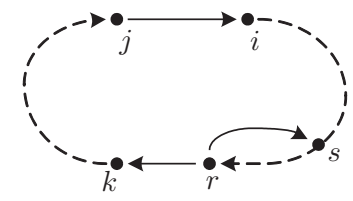

Figure 4.4: Described situation in $g^{\prime \prime}$, where $s$ is located on the directed path from $k$ to $r$.

This directed path also exists in $g^{\prime \prime}$, and together with $(r, k)$ it forms a cycle in $g^{\prime \prime}$ with $(r, s)$ being a spoke of it (see Figure 4.4). By deletion of $(r, s)$ we obtain a 
beneficial network

$$
g^{\prime \prime \prime}=g^{\prime \prime}-(r, s),
$$

due to BS. Its connection number is higher than the one of $g$. Observe that $g^{\prime \prime \prime}$ is proper: agents $j$ and $r$ (the only agents whose outgoing links are changed w.r.t. $g$ ) both have exactly one outgoing link in $g^{\prime \prime \prime}$. This is in contradiction with our assumption that $g$ is a proper network with maximal connection number. We conclude that there are no strictly improving additions available, i.e. $g$ is local-Nash.

When we relate the previous theorem with Theorem 4.2.2 (proper local-Nash networks are global-Nash if the joint payoff function satisfies DA, NA, and DE) and with the observation that $\mathbf{B T}$ and $\mathbf{B F}$ are implied by $\mathbf{D E}$, we obtain the following corollary.

Corollary 4.3.2. For joint payoff functions that satisfy $\boldsymbol{D A}, \boldsymbol{N A}, \boldsymbol{D E}, \boldsymbol{B} \boldsymbol{G}$, and $\boldsymbol{B S}$, global-Nash networks exist. Specifically, the proper and beneficial networks with maximal connection number are global-Nash.

\subsection{Architecture of (strict) local-Nash networks}

In this section, we study the architecture of local-Nash networks. In the previous section we proved existence of proper local-Nash networks for orderly joint payoff functions. However, proper networks are not the only candidates for local-Nash networks. Consider for instance the joint B\&G-1 function of Example 2.2.4 $\pi_{i}(g)=\left|N_{i}(g)\right|-\left|N_{i}^{d}(g)\right|$ for all $i \in N$. This function is orderly as we will see in Section 4.6. It is easily verified that the non-proper network depicted in Figure 2.2 is local-Nash. However, this network is not strict local-Nash, because the replacement of $(i, j)$ by $(k, j)$ yields the same payoff for agent $j$. When agent $i$ successively deletes the spoke $(k, i)$, we obtain a strict local-Nash network, which is also proper.

Moreover, any network can be local-Nash for payoff functions that satisfy all our properties, for instance when $\pi_{i}(g)=0$ for each agent $i$ and each network $g$. We will therefore focus on the architecture of strict local-Nash networks. In the next result, we characterize these networks.

Recall from Chapter 2 that network $g$ is called minimal if for each link $(j, i) \in g, j$ is not observed by $i$ in network $g-(j, i)$.

Theorem 4.4.1. Let $\pi$ be an orderly joint payoff function, and let $g$ be a strict localNash network. Then

(i) $g$ is a minimal network;

(ii) for all agents $i, j$, and $k$ where $(i, j) \in g$ and $(i, k) \in g$, there are no topagents active in $g_{-j}^{k}$;

(iii) each agent is contained in at most one cycle. 
Proof.

(i) Suppose to the contrary that a network, say $g$, exists which is strict local-Nash, while it is not minimal. Then a directed path from $k$ to $i$ exists in $g$ and also, the link $(k, i)$. By NA it follows directly that agent $i$ can delete link $(k, i)$, which yields an equal or higher payoff; a contradiction.

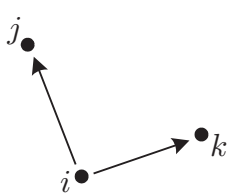

Figure 4.5: The situation of two outgoing links.

(ii) The situation where $(i, j)$ and $(i, k)$ exist is depicted in Figure 4.5. Suppose to the contrary that there are topagents active in component $g_{-j}^{k}$. Then by $\mathbf{B T}$, for at least one of these topagents, say agent $\ell$, the replacement of $(i, j)$ by $(\ell, j)$ is a good local response. Hence $g$ is not strict local-Nash, and therefore we conclude that there are no topagents active in $g_{-j}^{k}$.

(iii) Suppose to the contrary that an agent $i$ exists who is contained in at least two different cycles in $g$, say $C$ and $C^{\prime}$. Suppose that $i$ has an outgoing link that is contained in both $C$ and $C^{\prime}$. By following the directed path on the union of $C$ and $C^{\prime}$ that starts with this link, we necessarily arrive at an agent where $C$ and $C^{\prime}$ diverge. Without loss of generality, we may assume that this agent is $i$. This situation is depicted in Figure 4.6. By $\mathbf{B F}$ it follows that the replacement of $(i, j)$ by $(\ell, j)$ is a good local response. This contradicts that $g$ is strict local-Nash.

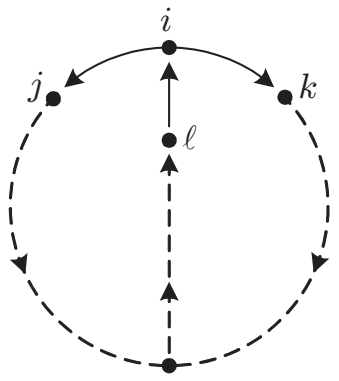

Figure 4.6: Situation where $i$ is contained in two cycles.

It remains an open question whether any network that satisfies the conditions (i), (ii), and (iii) of Theorem 4.4.1 is a Nash network for some orderly joint payoff function.

Notice that besides proper networks, not many network architectures satisfy those conditions. By (ii), it follows that for each agent $i$ who has two outgoing links, $(i, j)$ 
and $(i, k)$, there are no topagents active in the components $g_{-j}^{k}$ and (by symmetry) $g_{-k}^{j}$. This implies that these components cannot be proper, which in turn implies that in each of these components, another agent exists who has (at least) two outgoing links, and so on. In the following example, a strict local-Nash network is provided that is not proper. Already observe how the network depicted in Figure 4.7 satisfies condition (ii) by its cyclic structure; in view of agent 1, there are no topagents active in the components $g_{-2}^{n}$ and $g_{-n}^{2}$, and by symmetry, the same argument applies to all other odd-numbered agents.

Example 4.4.2. For even $n$ and $n \geq 4$, let $\tilde{g}$ be the following network architecture. Let all agents be contained in one undirected cycle, where the directions of the links are alternated. In Figure 4.7, network $\tilde{g}$ is depicted for $n=14$.

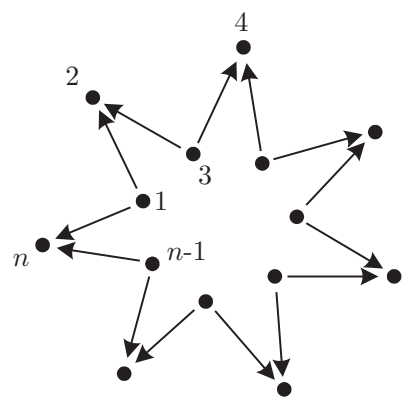

Figure 4.7: Example of network $\tilde{g}$.

For each $i \in N$, define $\pi_{i}(g)=1$ whenever $g=\tilde{g}$, and $\pi_{i}(g)=0$ otherwise. Since network $\tilde{g}$ is the unique network for which each agent yields a payoff strictly higher than 0 , it is a strict local-Nash network.

Now we show that $\pi$ is orderly.

DA is satisfied, since $\tilde{g}$ cannot be the union of two $i$-disjoint networks, and it is not $i$-disjoint from any non-empty network.

Property NA is also satisfied, because of the following. For any network $g$ where a directed path of at least two links exists from $k$ to $i$, it follows that $\pi_{i}(g+(k, i))=$ $\pi_{i}(g)=0$.

Since $\pi_{i}\left(g_{-i j}\right)=\pi_{i}\left(g_{-i}^{j}\right)=0$ for any network $g$ and any $i, j \in N$, links are always beneficial. Therefore, BF, BS, and BG are trivially satisfied. Further, since all links are equally beneficial, property $\mathbf{B T}$ is also satisfied.

Observe that network $\tilde{g}$ satisfies the conditions (i), (ii), and (iii) of Theorem 4.4.1. Therefore, and because $\pi$ is orderly, this example confirms Theorem 4.4.1 


\subsection{Property independence}

In this section, we show the independence of the properties that we used in this chapter. This is done by an exposition of examples of payoff functions, fulfilling all but one property.

Theorem 4.5.1. The properties $\boldsymbol{D A}, \boldsymbol{N A}, \boldsymbol{B} \boldsymbol{T}, \boldsymbol{B F}, \boldsymbol{B} \boldsymbol{G}$, and $\boldsymbol{B} \boldsymbol{S}$ are independent of each other.

Proof. We show that for each property a payoff function exists which does not satisfy that property while it does satisfy all other properties.

(all but DA) The following payoff function satisfies all properties, except DA:

$$
\pi_{i}(g)=\left|N_{i}(g) \backslash\{i\}\right|^{2} .
$$

Property DA is not satisfied, because for any two $i$-disjoint networks $g$ and $g^{\prime}$ we have $\left|N_{i}(g) \backslash\{i\}\right|^{2}+\left|N_{i}\left(g^{\prime}\right) \backslash\{i\}\right|^{2}<\left|N_{i}\left(g \cup g^{\prime}\right) \backslash\{i\}\right|^{2}$. The properties NA and BT are trivially satisfied and the others because $\pi_{i}\left(g_{-i j}\right) \geq \pi_{i}\left(g_{-i}^{j}\right)$ for any network $g$ and any agent $j$.

(all but NA) The following payoff function satisfies all properties, except NA:

$$
\pi_{i}(g)=\left|N_{i}^{d}(g)\right|
$$

Property NA is not satisfied, because $\pi_{i}(g+(k, i))>\pi_{i}(g)$ for any network $g$ where $(k, i) \notin g$, and where a directed path from $k$ to $i$ exists. Property DA is clearly satisfied, and also the four properties that concern beneficiality, because $\pi_{i}\left(g_{-i}^{j}\right)=0$ and $\pi_{i}\left(g_{-i j}\right)=1$ for any network $g$ and any agent $j$.

(all but BT) Let agent 1 be a special member of $N$, and let $i \in N \backslash\{1\}$. The following payoff function satisfies all properties, except BT:

$$
\pi_{i}(g)= \begin{cases}1 & \text { if } 1 \in N_{i}^{d}(g) \text { and } 1 \notin N_{i}(g-(1, i)) \\ 0 & \text { otherwise }\end{cases}
$$

This payoff function does not satisfy BT, because in any network $g_{-i}^{1}$ where agent 1 is not a topagent, we have $\pi_{i}\left(g_{-i 1}\right)=1$, while $\pi_{i}\left(g_{-i j}\right)=0$ for each topagent $j \in \operatorname{Car}\left(g_{-i}^{1}\right) \backslash\{1\}$. It can easily be verified that properties $\mathbf{N A}$ and DA are satisfied. The remaining properties are also satisfied since $\pi_{i}\left(g_{-i}^{j}\right)=0$ and $\pi_{i}\left(g_{-i j}\right) \geq 0$ for any network $g$ and any agent $j$. 
(all but BF) Let agent 1 be a special member of $N$. Consider the following payoff function:

$$
\pi_{i}(g)= \begin{cases}0 & \text { if } N_{i}(g)=N, 1 \notin N_{i}^{d}(g),\left|N_{i}^{d}(g)\right|=1 ; \\ -\left|N_{i}^{d}(g)\right| & \text { otherwise. }\end{cases}
$$

Observe that $\pi_{i}\left(g_{-i}\right)=0$ for any network $g$.

Property BF is not satisfied for $i \neq 1$, because of the following. Let $i \neq 1$ and let $g$ be a network that consists of one cycle containing all agents in $N \backslash\{i\}$, and that also consists of link $(i, k)$ for some $k \in N \backslash\{i, j\}$. Further, let 1 be the agent who is farthest away from $i(\mathrm{so},(1, k) \in g)$. Then $\pi_{i}\left(g_{-i k}\right)=0$ while $\pi_{i}\left(g_{-i 1}\right)=-1$.

It can easily be verified that property DA is satisfied. Property NA is satisfied, because if agent $i$ adds link $(j, i)$ to network $g$ where a directed path from $j$ to $i$ exists, then his payoff will decrease. Property BT is satisfied because only link $(1, i)$ can be beneficial, which is the case only if $N_{i}\left(g_{-i 1}\right)=N$, which implies that 1 is a topagent. By similar reasoning, properties BG and BS are satisfied.

(all but BG) The following payoff function satisfies all properties, except $\mathbf{B G}$ :

$$
\pi_{i}(g)=\left|N_{i}^{d}(g) \cap T\left(g_{-i}\right)\right|-\left|N_{i}^{d}(g) \backslash T\left(g_{-i}\right)\right|,
$$

where $T\left(g_{-i}\right)$ is the set of topagents in $g_{-i}$ who do not have outgoing links.

This payoff function does not satisfy BG, because of the following example. Consider network $g=\{(r, k),(k, i)\}$. Since $k \in T\left(g_{-i}^{k}\right)$, we have $\pi_{i}\left(g_{-i k}\right)=1$ and $\pi_{i}\left(g_{-i}^{k}\right)=0$, i.e. $(k, i)$ is beneficial in $g$. However, by adding link $(k, r)$, we obtain $k \notin T\left((g+(k, r))_{-i}^{k}\right)$. Therefore $\pi_{i}\left((g+(k, r))_{-i k}\right)=-1$ and $\pi_{i}\left((g+(k, r))_{-i}^{k}\right)=$ 0, i.e. $(k, i)$ is not beneficial in $g+(k, r)$. Hence property $\mathbf{B G}$ is not satisfied.

Property DA is satisfied, because the sets $N_{i}^{d}(g)$ and $T(g)$ can be decomposed into disjoint subsets w.r.t. $i$-disjoint subnetworks. Property NA is satisfied, because in any network $g$ where a directed path exists from $k$ to $i$, we have $k \notin T(g)$, and therefore the payoff does not increase when $(k, i)$ is added. Property BT is trivially satisfied since $(k, i)$ can only be beneficial if $k$ is a topagent in $g_{-i}$. Furthermore, a topagent $k$ in $g_{-i}$ such that $(k, i)$ is beneficial, has no outgoing links in $g_{-i}$ (since $k \in T\left(g_{-i}\right)$ ). If $g$ is proper, and if $i$ has an outgoing link to the component $g_{-i}^{k}$, then it follows that $k$ is the agent who is farthest away from $i$ in $g$. Hence BF is also satisfied. Property BS is satisfied because the deletion of a spoke $(k, r)$ in $g$ does neither affect the set $T\left(g_{-i}\right)$ nor the set $N_{i}^{d}(g)$.

(all but BS) The following payoff function satisfies all properties, except BS:

$$
\pi_{i}(g)=\left|K_{i}(g)\right|-\left|N_{i}^{d}(g)\right|,
$$


where $K_{i}(g)$ is the set of spokes that $i$ views in $g$, i.e.

$$
K_{i}(g)=\left\{(k, r): r \in N_{i}(g) \text { and }(k, r) \text { is a spoke }\right\} .
$$

Property BS is not satisfied, because by removing a spoke $(k, r)$ in a network $g$, the cardinality of $K_{i}(g)$ may decrease such that $\pi_{i}\left(g_{-i j}\right) \geq \pi_{i}\left(g_{-i}^{j}\right)$ and $\pi_{i}((g-$ $\left.(k, r))_{-i j}\right)<\pi_{i}\left((g-(k, r))_{-i}^{j}\right)$. Clearly, this payoff function satisfies $\mathbf{N A}$ and DA. For the properties BT, BF, and BG, notice that the payoff $\pi_{i}\left(g_{-i j}\right)$ only depends on the number of spokes viewed in $g_{-i j}$. Properties BT and BF are satisfied, because of the following. Let $k$ be an agent in a network $g$ and let $j$ be a topagent in $g_{-i}^{k}$. Since $i$ views at least as many spokes in $g_{-i j}$ as in $g_{-i k}$, BT and $\mathbf{B F}$ are satisfied. Property BG is satisfied because for any network $g$ and any agent $j$, the number $\left|K_{i}\left(g_{-i j}\right)\right|$ cannot decrease by adding a link to $g$.

We already observed that $\mathbf{D E}$ implies $\mathbf{B T}$ and $\mathbf{B F}$, and not vice versa. In the following theorem, we show that the properties that are needed for Corollary 4.3 .2 which are NA, DA, DE, BS, and BG, are independent of each other as well.

Theorem 4.5.2. The properties $\boldsymbol{D A}, \boldsymbol{N A}, \boldsymbol{D E}, \boldsymbol{B} \boldsymbol{G}$, and $\boldsymbol{B} \boldsymbol{S}$ are independent of each other.

Proof. By Theorem 4.5.1 we know that DA, NA, BG, and BS are independent of each other. Therefore it remains to show that DA, NA, BG, and BS are independent of DE.

Payoff function (4.7) in Example 4.2.3 does not satisfy DE, whereas it satisfies all other properties. In the example it was shown that DA and NA are satisfied. The other properties, $\mathbf{B G}$ and $\mathbf{B S}$, are trivially satisfied since $\pi_{i}\left(g_{-i}^{j}\right)=0$ and $\pi_{i}\left(g_{-i j}\right)=1$ for any $g$ and $j$. Hence NA, DA, BG, and BS do not imply DE.

To show that DE does not imply DA, NA, BG, nor BS, consider the payoff functions 4.11, 4.12, 4.15), and 4.16). They do not satisfy DA, NA, BG, and BS respectively. However, it can easily be checked that these functions do satisfy DE.

We conclude that the properties DA, NA, DE, BG, and BS are independent of each other.

\subsection{Payoff functions satisfying the properties}

In this section, we analyze which payoff functions satisfy the framework of properties that we introduced in this chapter. First, we focus on B\&G-1 functions (defined by $(2.2)$ ). We prove that B\&G-1 functions with owner-homogeneous link costs and heterogeneous profits are orderly and also satisfy DE. Further, we prove that B\&G-1 functions with heterogeneous link costs that satisfy a system of triangle inequalities, 
are orderly without necessarily satisfying DE. Then, we provide several examples of payoff functions that satisfy all properties, even though they fall outside the class of B\&G-1 functions.

Recall from Section 2.2 .2 that we may assume that $v_{i i}=0$, because the transformation $\pi_{i}^{\prime}(g)=\pi_{i}(g)-\pi_{i}\left(g_{-i}\right)=\pi_{i}(g)-v_{i i}$ does not have influence on the strategic behavior of agent $i$.

In the next lemma, we prove that all B\&G-1 functions satisfy four properties.

Lemma 4.6.1. Let $\pi_{i}$ be a BEG-1 function. Then $\pi_{i}$ satisfies $\boldsymbol{D A}, \boldsymbol{N A}, \boldsymbol{B} \boldsymbol{G}$, and $B S$.

Proof.

(DA) For each two $i$-disjoint networks $g$ and $g^{\prime}$ it holds that $N_{i}(g) \cap N_{i}\left(g^{\prime}\right)=\{i\}$ and $N_{i}^{d}(g) \cap N_{i}^{d}\left(g^{\prime}\right)=\emptyset$. Since we assumed that $v_{i i}=0$, it follows that $\pi_{i}\left(g+g^{\prime}\right)=$ $\pi_{i}(g)+\pi_{i}\left(g^{\prime}\right)$. Therefore $\pi_{i}$ satisfies DA.

(NA) If a directed path exists from $k$ to $i$ in network $g$ where link $(k, i)$ does not exist, then $N_{i}(g)=N_{i}(g+(k, i))$, and $N_{i}^{d}(g) \subset N_{i}^{d}(g+(k, i))$. Hence property $\mathbf{N A}$ is satisfied.

(BG) Let $g$ be a network where $(j, i)$ is beneficial. Since $N_{i}\left(g_{-i j}\right) \subseteq N_{i}\left((g+(k, r))_{-i j}\right)$ and $N_{i}^{d}\left(g_{-i j}\right)=N_{i}^{d}\left((g+(k, r))_{-i j}\right)=1$, property $\mathbf{B G}$ is satisfied.

(BS) Let $g$ be a network that contains a spoke $(k, r)$. Let $(j, i)$ be beneficial in $g$. Since $N_{i}\left(g_{-i j}\right)=N_{i}\left((g-(k, r))_{-i j}\right)$ and $N_{i}^{d}\left(g_{-i j}\right)=N_{i}^{d}\left((g-(k, r))_{-i j}\right)=1$, link $(j, i)$ is also beneficial in $g-(k, r)$. Hence $\mathbf{B S}$ is satisfied.

In the next result, we show that B\&G-1 functions with owner-homogeneous link costs satisfy all properties, and therefore imply the existence of global-Nash networks (by Corollary 4.3.2). This result is also proved in Theorems 2.2.2 and 2.2.8.

Theorem 4.6.2. Let $\pi$ be a joint BEG-1 function with owner-homogeneous link costs, i.e. $c_{i j}=c_{i}$ for all $i, j \in N$. Then $\pi$ satisfies $\boldsymbol{D A}, \boldsymbol{N} \boldsymbol{A}, \boldsymbol{D E}, \boldsymbol{B} \boldsymbol{G}$, and $\boldsymbol{B} \boldsymbol{S}$, i.e. global-Nash networks exist.

Proof. By Lemma 4.6.1 it follows that $\pi$ satisfies DA, NA, BG, and BS.

Let $g$ be a network where $(j, i) \notin g,(k, i) \notin g$, and where a directed path exists from $k$ to $j$ in $g_{-i}$. Then $N_{i}(g+(j, i)) \supseteq N_{i}(g+(k, i))$ and $\left|N_{i}^{d}(g+(j, i))\right|=\left|N_{i}^{d}(g+(k, i))\right|$. Hence, property DE is satisfied.

The existence of local-Nash networks is proved in Theorem 4.3.1 for orderly payoff functions. Notice that these payoff functions satisfy BT and BF instead of DE (which implies both of them). In the next theorem we provide conditions for joint B\&G-1 functions with heterogeneous link costs such that these functions are orderly. 
Theorem 4.6.3. Let $\pi$ be a joint B\&G-1 function with heterogeneous link costs and profits. If

$$
c_{i j} \leq v_{i j}+\min \left(v_{i k}, c_{i k}\right), \text { for all } i, j, k \in N
$$

then $\pi$ is orderly, i.e. local-Nash networks exist.

Proof. By Lemma 4.6.1, the properties DA, NA, BG, and BS are satisfied. It remains to prove that $\pi_{i}$ satisfies $\mathbf{B T}$ and $\mathbf{B F}$ :

(BT) Let link $(k, i)$ be beneficial in $g$. Then $\sum_{r \in N_{i}\left(g_{-i k}\right)} v_{i r} \geq c_{i k}$. If a topagent $j$ exists in the component $g_{-i}^{k}$, then either $k=j$ or a directed path from $k$ to $j$ exists. In the first case $\mathbf{B T}$ is trivially satisfied. In the second case, it follows that $N_{i}\left(g_{-i j}\right) \supseteq N_{i}\left(g_{-i k}\right) \cup\{j\}$. Since $c_{i j} \leq v_{i j}+c_{i k}$ we have

$$
\begin{aligned}
\pi_{i}\left(g_{-i k}\right) & =\left(\sum_{r \in N_{i}\left(g_{-i k}\right)} v_{i r}\right)-c_{i k} \\
& \leq\left(\sum_{r \in N_{i}\left(g_{-i k}\right)} v_{i r}\right)-\left(c_{i j}-v_{i j}\right) \\
& \leq\left(\sum_{r \in N_{i}\left(g_{-i j}\right)} v_{i r}\right)-c_{i j} \\
& =\pi_{i}\left(g_{-i j}\right) .
\end{aligned}
$$

Hence BT is satisfied by $\pi_{i}$.

(BF) Let $g_{-i}^{k}$ be a proper component of $g$ where $i$ has an outgoing link and let link $(k, i)$ be beneficial in $g$. Let $j$ be a topagent in this component who is farthest away from $i$. If $k=j$ then $\mathbf{B F}$ is trivially satisfied. Otherwise a path from $k$ to $j$ exists. Therefore both agents $j$ and $k$ are contained in $N_{i}\left(g_{-i j}\right)$. Since $c_{i j} \leq v_{i j}+v_{i k}$ it follows that $\pi_{i}\left(g_{-i j}\right) \geq v_{i j}+v_{i k}-c_{i j} \geq 0$. Hence $\mathbf{B F}$ is satisfied.

Observe that both local- and global-Nash networks also exist for B\&G-1 functions under weaker conditions than 4.17). This is illustrated by Proposition 2.2.10. Notice that a B\&G-1 function that fulfills (2.9) and 2.10) does not necessarily satisfy BF. In Chapter 6 we return to this subject more extensively: we provide a full characterization of B\&G-1 functions that satisfy the properties of our framework, and hence imply the existence of local- and global-Nash networks.

Our framework of properties is also satisfied by non-B\&G-1 payoff functions. Consider the following examples:

$$
\begin{aligned}
& \pi_{i}(g)=\left|N_{i}^{d}(g) \cap T\left(g_{-i}\right)\right|, \\
& \pi_{i}(g)=\left|K_{i}(g) \backslash\left\{(j, i): j \in N_{i}^{d}(g)\right\}\right|, \\
& \pi_{i}(g)=\left|C(g) \cap N_{i}(g)\right|-\left|N_{i}^{d}(g)\right|,
\end{aligned}
$$

where $C(g)$ is the set of agents that are contained in a cycle in $g$, and where $T(g)$ and $K_{i}(g)$ are respectively defined as the set of topagents in $g$ who do not have outgoing 
links, and the set of spokes in $g$ that $i$ observes. These payoff functions are orderly and also satisfy DE. Payoff function 4.7) in Example 4.2.3, which is also studied in the proof of Theorem 4.5.2, is a non-B\&G-1 payoff function that is orderly whereas it does not satisfy DE.

These payoff functions extend the class of B\&G-1 functions in the following way. They do not only consider which agents are (directly) observed, i.e. which agents are contained in the sets $N_{i}(g)$ and $N_{i}^{d}(g)$. They also take other aspects of the network architecture into account. In the given examples, the sets $T\left(g_{-i}\right), K_{i}(g)$, and $C(g)$ illustrate this.

Bala and Goyal (2000a) study a class of payoff functions that generalizes B\&G-1 functions with homogeneous links costs and homogeneous profits:

$$
\pi_{i}(g)=\phi\left(\left|N_{i}(g)\right|,\left|N_{i}^{d}(g)\right|\right) \quad \forall i \in N,
$$

where $\phi(x, y)$ is strictly increasing in $x$ and strictly decreasing in $y$. Observe that only the number of observed agents and the number of own links determine the payoff. In other words, the observed agents and the links are still regarded as homogeneous. It can be easily verified that these functions satisfy all proposed properties, except DA. It remains an open question whether the existence of Nash networks can be proved for payoff functions that allow for heterogeneity among observed agents and link costs, without using a linear type of property, such as DA.

\subsection{Concluding remarks}

In conclusion, we studied the existence of Nash networks in this chapter for a general class of payoff functions. The local oriented payoff properties that we introduced provide insights in the specific underlying model (in this case, the one-way flow model): which properties are needed to prove the existence of Nash networks, and how each property affects the architecture of those networks. For instance, by NA it follows that minimal networks are preferred over non-minimal networks.

The payoff functions that satisfy the framework of properties include B\&G-1 functions with owner-homogeneous link costs and heterogeneous profits, and non-B\&G-1 functions such as 4.18, 4.19, and 4.20). As far as we know, there is no natural class of non-B\&G-1 functions that satisfies the framework of properties.

Non-linear payoff functions such as 4.21 do not satisfy DA. For further research it is worthwhile to develop a framework of payoff properties that also cover non-linear payoff functions.

In Chapter 7, we analyze the two-way flow model in the same way; we introduce a framework of payoff properties which are inspired by the two-way flow model, and which provide insights in the two-way flow model. In Chapter 9 we discuss both frameworks and relate them to models with information decay and imperfectly reliable links. 


\section{Chapter 5}

\section{Dynamic network formation game}

In this chapter, we study a dynamic game of network formation based on the model studied in the previous chapter.

The game starts with some initial network. Then one agent, who is selected at random, plays a local action, i.e. he can modify the network by adding, deleting, or replacing a link, but he can also pass. Then again, one agent is selected to play a local action in the obtained network. This process continues, until a network is reached that no one wants to modify. This network yields a payoff for each agent. The goal of each agent is to obtain a payoff as high as possible.

Our approach of local dynamics reflects a local oriented strategic behavior of the agents. By playing local improvements, agents improve their current situation by a minimal modification, which is a realistic aspect in many applications of network formation. Another advantage of our local approach is that a best local response is much easier to find in terms of computational complexity than a best global response (see Theorem 3.2.1 and Proposition 4.1.1.

In this chapter we analyze how this game is played. We want to know whether the play ends. In other words, does the play lead to a network that no one wants to modify? For this purpose, we propose an iterative procedure of local actions.

This procedure follows the rules of the game as described above. Thus, starting with some initial network, at the beginning of each stage, one agent is selected to modify the network. Then, one of his local actions is selected at random and being played. We allow agents to play more actions than best local responses. Also actions are allowed where the obtained payoff does not decrease. We call these actions good local responses. These actions can be seen as ad-hoc improvements, which are realistic in applications of network formation, especially with respect to large networks. All good local responses (except for some specific payoff equivalent local responses) have 
a positive fixed probability to be selected, that only depends on the network.

We say that our procedure terminates when it reaches a network that no agent wants to modify. We prove that if all payoff properties of chapter 4 (introduced in Sections 4.2 and 4.3) are satisfied, then the procedure terminates in a finite number of iterations. Moreover, the networks at which the procedure terminates, are global-Nash networks.

Our procedure resembles the one described by Bala and Goyal (2000a). However, they examine only global actions, and multiple agents may play simultaneously during each stage of their model. In the last section of this chapter we show that our results can be generalized to the case where agents may play simultaneously. Watts (2001) and Jackson and Watts (2002) also study dynamic models of network formation, but their models are based on the bilateral model of network formation introduced by Jackson and Wolinsky (1996).

This chapter is outlined as follows. In Section 5.1 we formally describe the dynamic game, and provide examples of how it can be played. Then, in Section 5.2 we propose the procedure and prove that it always terminates at a global-Nash network whenever all payoff properties are satisfied. Finally, in Section 5.3 we provide several concluding remarks.

This chapter is mainly based on Derks et al. (2008b).

\subsection{Game description}

Given a set of agents $N$ and a joint payoff function $\pi$, a network formation game proceeds in stages $1,2,3, \ldots$. Let $g_{t}$ be the network at the beginning of stage $t$, which is known to all agents. The initial network $g_{1}$ can be any network in $\mathcal{G}$. Then, at stage $t$ according to a probability device, an agent, say $i$, is selected. We assume that at each stage all agents have positive (stage independent) probabilities of being selected. Now, stage $t$ proceeds by allowing one agent to modify the network $g_{t}$ by playing a local action. Thus, a new network $g_{t+1}$ results, which marks the start of stage $t+1$. The game ends with network $g_{*}$ if no agent wants to adjust his links. In that case, each agent $i$ receives payoff $\pi_{i}\left(g_{*}\right)$.

Recall that a local action is one of the four following types: a pass, an addition, a deletion, and a replacement. Further, recall that a local action of agent $i$ in stage $t$ where the obtained network is $g_{t+1}$, is called a best local response if

$$
\pi_{i}\left(g_{t+1}\right) \geq \pi_{i}\left(\left(g_{t}\right)_{-i}+\{(j, i): j \in T\}\right),
$$

for all local actions $T$; a good local response if

$$
\pi_{i}\left(g_{t+1}\right) \geq \pi_{i}\left(g_{t}\right),
$$

and, in addition, a neutral local response if

$$
\pi_{i}\left(g_{t+1}\right)=\pi_{i}\left(g_{t}\right) .
$$


The following example with three agents illustrates how the network formation game is played.

Example 5.1.1. Let the set of agents be $N=\{1,2,3\}$ and for each agent $i \in N$, let $\pi_{i}$ be a B\&G-1 function as described in (2.2), where $v_{i j}=2$ and $c_{i j}=1$ for all agents $j$.

\begin{tabular}{lllllll}
\hline Stage $t$ & Network $g_{t}$ & $\pi_{1}\left(g_{t}\right)$ & $\pi_{2}\left(g_{t}\right)$ & $\pi_{3}\left(g_{t}\right)$ & $\begin{array}{l}\text { Selected } \\
\text { agent }\end{array}$ & Local action \\
\hline 1 & $g_{1}=\emptyset$ & 0 & 0 & 0 & 1 & add $(3,1)$ \\
2 & $g_{2}=\{(3,1)\}$ & 1 & 0 & 0 & 3 & add $(1,3)$ \\
3 & $g_{3}=\{(3,1),(1,3)\}$ & 1 & 0 & 1 & 2 & add $(3,2)$ \\
4 & $g_{4}=\{(3,1),(1,3),(3,2)\}$ & 1 & 3 & 1 & 1 & replace $(3,1)$ with $(2,1)$ \\
5 & $g_{5}=\{(2,1),(1,3),(3,2)\}$ & 3 & 3 & 3 & All agents pass. \\
\hline
\end{tabular}

Table 5.1: Play of the game in Example 5.1.1.

Let the initial network in this example, $g_{1}$, be the empty network. The play of the game is shown in Table 5.1. In the second last column the selected agent is given, but the agents do not know the order in which they are chosen in advance. The corresponding networks are depicted in Figure 5.1. Notice that all played local actions in this example are best local responses. Furthermore, the final network, $g_{5}$, is strict local- and global-Nash.
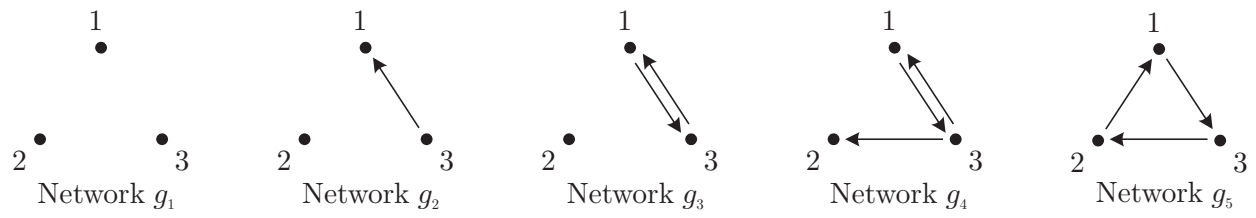

Figure 5.1: The networks obtained in Example 5.1.1.

In this chapter we study the play of this game. We say that the play terminates at a specific network $g_{*}$ if this network is reached, and furthermore, if no agent exists who wants to play a local action that modifies $g_{*}$. Clearly, the play described in Example 5.1.1 has terminated at network $g_{5}$. However, by the next example we will see that termination is not evident. Here, we will revisit the game described in Example 2.2.9. for which Nash networks do not exist.

Example 5.1.2. For this game, let $N=\{1,2,3,4\}$. Let $\pi$ be a joint B\&G-1 function. The profits are given by $v_{i j}=1$ for all $i, j$ with $i \neq j$ and $v_{i i}=0$ for all $i$. Further, the numbers next to the links in Figure 5.2 indicate the costs of these links. Here, $\epsilon$ is a strictly positive number which can be chosen arbitrarily close to 0 .

The costs of the links that are not depicted in this figure are the following: 


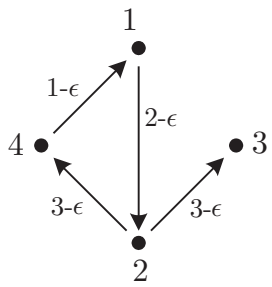

Figure 5.2: The link costs.

* links directed to agent 1 have cost $1+\epsilon$,

* links directed to agent 2 have cost $2+\epsilon$,

* links directed to agents 3 and 4 have cost $3+\epsilon$,

Suppose that the play reaches the network $g_{t}$ depicted in Figure 5.3 at a certain stage $t$. The play of the game as from stage $t$ is given in Table 5.2 and the obtained networks are depicted in Figure 5.3. For the sake of convenience, we skip the possible intermediate stages in which agents pass.

\begin{tabular}{llllllll}
\hline Stage & Network & $\pi_{1}$ & $\pi_{2}$ & $\pi_{3}$ & $\pi_{4}$ & Agent who can improve & Local action \\
\hline $\mathrm{t}$ & $g_{t}$ & $1+\epsilon$ & $\epsilon$ & $\epsilon$ & $\epsilon-1$ & 4 & delete $(2,4)$ \\
$\mathrm{t}+1$ & $g_{t+1}$ & $\epsilon$ & $\epsilon$ & $\epsilon$ & 0 & 1 & add $(3,1)$ \\
$\mathrm{t}+2$ & $g_{t+2}$ & 1 & $1+\epsilon$ & $\epsilon$ & 0 & 4 & add $(2,4)$ \\
$\mathrm{t}+3$ & $g_{t+3}$ & 1 & $1+\epsilon$ & $\epsilon$ & $\epsilon$ & 1 & delete $(3,1)$ \\
$\mathrm{t}+4$ & $g_{t}$ & & & & & & \\
\hline
\end{tabular}

Table 5.2: Play of the game in Example 5.1.2.

Since agents 1,2 , and 3 play optimally in network $g_{t}$, agent 4 is the only one who wants to modify the network by deleting $(2,4)$, obtaining network $g_{t+1}$. In this network, agent 1 is the only one who can improve, namely by adding $(3,1)$. In the obtained network $g_{t+2}$, agent 4 can improve by adding $(2,4)$ such that network $g_{t+3}$ is obtained. Next, agent 1 can improve by deleting $(3,1)$. The obtained network $g_{t+4}$ is equal to $g_{t}$. Hence, the dynamic game cycles through the networks depicted in Figure 5.3 . $\diamond$

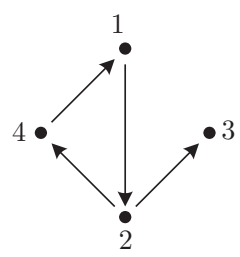

$g_{\mathrm{t}}$

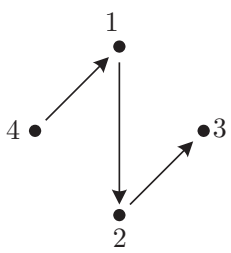

$g_{t+1}$

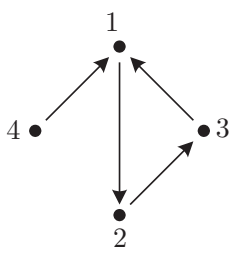

$g_{t+2}$

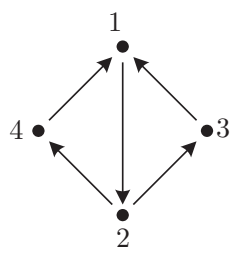

$g_{t+3}$

Figure 5.3: The networks obtained in Example 5.1.2. 


\subsection{Procedure}

In this section we study how the game is played by means of a dynamic procedure. We prove that this procedure terminates at a proper global-Nash network, whenever all properties of the previous chapter are satisfied. Here, we make use of the results from chapter 4 , in particular the proof of Theorem 4.3.1. The procedure that we propose is completely in line with those results.

Following the rules of the game, the procedure starts with an arbitrary initial network, and per stage, one agent is selected at random. One of his good local responses is selected at random, and being played. These steps are repeated. Formally, we define the procedure based on the following assumptions.

A-1 Let the initial network be a network that is arbitrarily chosen from $\mathcal{G}$.

A-2 At the beginning of each stage, an agent is selected at random, where each agent has a positive stage independent probability to be selected.

A-3 At each stage, the agent who is selected plays a good local response that satisfies the following three assumptions.

A-3a A neutral addition is not allowed.

A-3 b A neutral deletion of link $(j, i)$ in network $g$ is only allowed whenever $N_{i}(g-(j, i))=N_{i}(g)$.

A-3 A neutral replacement of $(k, i)$ by $(j, i)$ in network $g$ is only allowed when a directed path exists from $k$ to $j$ in network $g_{-i}$.

One of his good local response is selected at random, where all allowed good local responses have a positive probability to be selected that only depends on the network.

We say that the procedure terminates at a network $g$ if this network is reached, and furthermore, if a pass is the only allowed action for each agent $i$ with respect to $g$.

Unfortunately, assumptions $\mathrm{A}-3 \mathrm{a}$ to $\mathrm{A}-3 \mathrm{c}$ are restrictive to some extent. It would be more intuitive that agents have more options to experiment with, for instance by adding links where their payoffs do no immediately increase. However, we need those assumptions in order to prevent situations like the following one. Consider a game where an agent $i$ is present, such that $\pi_{i}(g)=0$ for any network $g$. When the procedure reaches some local-Nash network, this agent may perform randomly chosen neutral local responses, such that the obtained network is no longer local-Nash. With these kind of payoff functions, it is hard (if not impossible) to design a more general procedure that terminates at a local-Nash network.

In the following lemma, we show that the procedure defined by A-1 to A- 3 terminates whenever a proper local-Nash network is reached. 
Lemma 5.2.1. Let the dynamic procedure be defined by assumptions $A-1$ to $A-3$. If this procedure reaches a proper local-Nash network, then it also terminates at this network.

Proof. Let $g$ be a proper local-Nash network that is reached by the dynamic procedure. Since $g$ is local-Nash, it can only be modified by neutral responses. Let $i$ be an agent who can apply a neutral response to $g$. We know by assumption A-3a that this action cannot be a neutral addition.

Suppose that this action is a deletion. Since $g$ is proper, each deletion strictly reduces the set of observed agents. By assumption $\mathrm{A}-3 \mathrm{~b}$, such deletions are not allowed. Hence we conclude that this action cannot be a deletion.

Suppose that this action is a replacement. By assumption $\mathrm{A}-3 \mathrm{c}$, a neutral replacement of $(k, i)$ by $(j, i)$ is only allowed when a directed path exists from $k$ to $j$ that does not visit agent $i$. In that case, agent $k$ has two outgoing links: $(k, i)$ and a link on the path from $k$ to $j$. This contradicts that $g$ is proper.

Hence we conclude that the only neutral response that can be applied to $g$ is a pass. Therefore, the procedure terminates at network $g$.

We prove that the procedure reaches a proper local-Nash network with probability 1 . First, we show that a finite sequence of good local responses exists that can be applied iteratively to any arbitrary network such that the obtained network is local-Nash. This sequence starts with actions such that the initial network is reshaped to a proper and beneficial network. From there, we reuse the result of the proof of Theorem 4.3.1 which states that if a proper and beneficial network is not local-Nash, then another proper and beneficial network exists with a higher connection number. Iteratively using this result, we obtain a network with a maximal connection number, which implies that this network is proper local-Nash.

Lemma 5.2.2. Let $\pi$ satisfy $\boldsymbol{D A}, \boldsymbol{N A}$, and $\boldsymbol{D E}$. Then, for any network in $\mathcal{G}$, there exists a finite sequence of good local responses that leads to a proper and beneficial network.

Proof. Let $g$ be a network in $\mathcal{G}$. In two steps, we provide a sequence of good local responses that starts at $g$, and that leads to a proper and beneficial network.

Step 1. First we make $g$ proper by applying good local responses. If $g$ is already proper, then continue to step 2. Otherwise an agent $i$ exists in $g$ who has at least two outgoing links, say $(i, j)$ and $(i, k)$ (see Figure 5.4.

Two cases are distinguished:

A: There is a directed path from $i$ to an agent $\ell$ with outdegree 0 , starting with link $(i, k)$. Property DE implies that the link $(i, j)$ may be replaced by $(\ell, j)$. This action decreases the total outdegree of the agents with multiple outgoing links. 


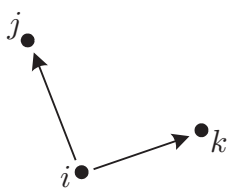

Figure 5.4: An agent with two outgoing links.

B: None of the directed paths starting with link $(i, k)$ end at an agent with outdegree 0 . Either there is a cycle $C$ containing $(i, k)$, or there is a directed path starting with link $(i, k)$ and ending at an agent $\ell$ on a cycle. In the latter case we may apply property $\mathbf{D E}$ and replace link $(i, j)$ with $(\ell, j)$. It is therefore no loss of generality to assume a cycle containing $(i, k)$.

We distinguish four subcases:

1: Agent $j$ is on cycle $C$. Then a directed path exists from $i$ to $j$ and hence the link $(i, j)$ can be deleted by NA.

2: There is a directed path from $i$ to an agent with outdegree 0 , and starting with link $(i, j)$. Case $\mathbf{A}$ addresses this situation.

3: There is a cycle $C^{\prime}$ containing $(i, j)$. Going in the opposite direction over $C^{\prime}$, let $\ell$ be the last agent on this cycle who is also on cycle $C$ (see Figure 5.5).

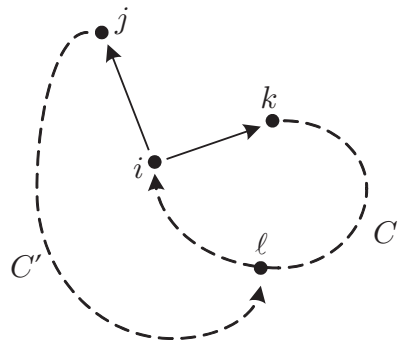

Figure 5.5: Situation of case 3 .

Using property DE we may replace link $(i, k)$ with link $(\ell, k)$, so that we can assume that both cycles $C$ and $C^{\prime}$ have only agent $i$ in common. This situation is depicted in Figure 5.6 .

Let agent $\ell$ be such that $(\ell, i)$ is on cycle $C(\ell$ may be agent $k)$. Now, replace $(i, j)$ with $(\ell, j)$. This is a good local response by $\mathbf{D E}$ since there is a directed path from $i$ to $\ell$, without visiting $j$. After this replacement, the link $(\ell, i)$ can be deleted by NA since there is a cycle in which $i, k, \ell, j$ are visited in this order, and hence $(\ell, i)$ is a spoke.

4: There is a directed path starting with link $(i, j)$ and ending at an agent $\ell$ on a cycle. Then agent $\ell$ is not on the cycle through $(i, k)$. (Otherwise, we would 


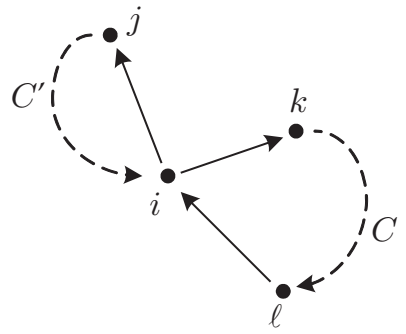

Figure 5.6: Situation of case 3 continued.

have obtained a cycle containing $(i, j)$, and this is already taken care of in case 3.) Using property $\mathbf{D E}$, we may replace link $(i, k)$ with link $(\ell, k)$, and by this action we arrive at the situation treated in case 3 .

As long as there are agents with outdegree greater than $1, g$ is not proper, and hence this step can be repeated. Each time it is repeated, the outdegree of one agent is reduced without changing the outdegrees of the other agents. Therefore, after a finite number of repetitions we obtain a proper network.

Step 2. Let $g^{\prime}$ be the proper network that results from step 1. If $g^{\prime}$ is not beneficial, then a non-beneficial link $(j, i)$ exists in $g^{\prime}$. Since $g^{\prime}$ is proper and since DA is satisfied, by Lemma 4.2.1 we know that $(j, i)$ is also not profitable, and therefore the deletion of it is a good local response. Obviously, $g^{\prime}$ remains proper after this deletion. Such deletions can be applied repeatedly until we obtain a proper and beneficial network $g^{\prime \prime}$.

Observe that also for a local-Nash network that is not proper, there exists a sequence that leads to a proper and beneficial network. The next lemma shows that for any proper and beneficial network, there exists a finite sequence of good local responses that can be applied iteratively such that it leads to a proper local-Nash network.

Lemma 5.2.3. Let $\pi$ be an orderly joint payoff function that satisfies $\boldsymbol{D E}$. Let $g$ be a proper and beneficial network. There exists a finite sequence of good local responses that leads to a proper local-Nash network.

Proof. Suppose that $g$ is not local-Nash. Since $g$ is proper and beneficial, we know from the proof of Theorem 4.3.1 that a network can be obtained with a higher connection number. We show that we can obtain this network by applying good local reponses.

Consider the networks $g^{\prime}, g^{\prime \prime}$ and $g^{\prime \prime \prime}$ as defined in 4.8, 4.9, and 4.10. Network $g^{\prime}$ is obtained from $g$ by a strictly improving addition, which is trivially a good local response. Network $g^{\prime \prime}$ is obtained from $g^{\prime}$ by a replacement of $(j, k)$ by $(r, k)$ where a directed path from $j$ to $r$ exists in $g$. By DE, this is also a good local response. Finally, network $g^{\prime \prime \prime}$ is obtained from $g^{\prime \prime}$ by a deletion of spoke $(r, s)$ which is a good local response by NA. Observe that $g^{\prime \prime \prime}$ is proper and benefical. Therefore, if $g^{\prime \prime \prime}$ is 
not local-Nash, we can repeat these good local responses until we obtain a local-Nash network. At each iteration, the connection number strictly increases. Since this number is bounded by $n(n-1)$, we obtain a local-Nash network in a finite number of iterations.

Combining Lemma's 5.2.2 and 5.2.3 we obtain a sequence of networks that starts with an arbitrary initial network and ends with a proper local-Nash network. In the next theorem we show that our procedure always terminates at a local-Nash network.

Theorem 5.2.4. Let $\pi$ be an orderly joint payoff function that satisfies $\boldsymbol{D E}$, and let the dynamic procedure be defined by assumptions $A-1$ to $A \sqrt{3}$. Then this procedure terminates at a proper local-Nash network with probability 1.

Proof. First we prove that the procedure reaches a local-Nash network with probability 1, and then we know by Lemma 5.2.1 that it also terminates at this network.

By Lemma's 5.2 .2 and 5.2 .3 we know that from an arbitrary network in $\mathcal{G}$ a finite sequence of good local responses exists, such that the obtained network is proper and local-Nash. It is easily verified that these good local responses satisfy assumptions $\mathrm{A}-3 \mathrm{a}$ to $\mathrm{A}-3 \mathrm{c}$

* the only additions in this sequence are strictly improving ones;

* each deletion is either validated as a good local response by NA (and hence it satisfies assumption $\mathrm{A}-3 \mathrm{~b}$, or it is a deletion of a non-beneficial link in a proper network which is a strictly improving deletion by $\mathbf{D A}$;

* all replacements in this sequence are validated as good local responses by DE and hence they satisfy assumption $\mathrm{A}-3 \mathrm{c}$.

Hence, any sequence that is constructed in the proofs of Lemma's 5.2.2 and 5.2.3 satisfies the assumptions $\mathrm{A}-1$ to $\mathrm{A}-3$

By the construction of such a sequence, we know that each network in $\mathcal{G}$ appears at most once in this sequence. Therefore, we conclude that the length of this sequence is upperbounded by $M=2^{n(n-1)}$, i.e. the finite number of networks in $\mathcal{G}$.

At any stage, each agent has a strictly positive probability to be selected (assumption A-2], and each allowed good local response has a strictly positive probability to be selected (assumption A-3. Therefore, the probability that such a sequence will be played is lowerbounded by a strictly positive probability $\epsilon$. The probability that the dynamic procedure does not reach a local-Nash network after $M$ steps is lower than $1-\epsilon$. If it does not reach a local-Nash network after $M$ steps, then from the last network, another sequence exists that leads to a local-Nash network. Hence, the probability that the dynamic procedure does not reach a local-Nash network after $2 M$ steps is lower than $(1-\epsilon)^{2}$, and after $k M$ steps lower than $(1-\epsilon)^{k}$, with $k$ being a strictly positive natural number. This probability converges to 0 as $k$ becomes larger. Therefore, the procedure reaches a proper local-Nash network with probability 1 . 
By Lemma 5.2.1 we know that this procedure also terminates at this network.

Combining this result with Theorem 4.2.2 saying that each proper local-Nash network is also global-Nash, we obtain the following corollary.

Corollary 5.2.5. Let $\pi$ be an orderly joint payoff function that satisfies $\boldsymbol{D E}$, and let the dynamic procedure be defined by assumptions $A-1$ to $A-3$. Then this procedure terminates at a proper global-Nash network with probability 1.

\subsection{Concluding remarks}

In our procedure, agents play good local actions, which are realistic in the sense that individuals link up with others in an ad-hoc way, rather than determining the best thing to do. They can also experiment by playing certain neutral local actions. However, if we allow agents to play all types of neutral local actions, then our procedure does not necessarily terminate. It would be interesting to study which classes of payoff functions imply the termination of our procedure without restrictions on neutral local actions (A-3a to $\mathrm{A}-3 \mathrm{c}$.

In our procedure, agents play in a sequential order (assumption A-2). Bala and Goyal (2000a) consider a more general order of play, where at each stage, each agent has a positive probability to be selected. This implies that agents can play simultaneously during each stage. Our restriction to a purely sequential order of play is not essential in order to prove termination. Consider the follow alternative assumption:

A-2' At the beginning of each stage, each agent has a positive, stage independent probability to be selected.

It can be proved as follows that the procedure defined by $\mathrm{A}-1, \mathrm{~A}-2,2$, and $\mathrm{A}-3$ (applied to each selected agent), terminates at a proper local-Nash network with probability 1 . For this purpose, the proof of Theorem 5.2.4 can be used, except for the first sentence of the second last paragraph, which should be replaced by: "At any stage and for each agent $i$, there is a strictly positive probability that $i$ is selected while all others are not (assumption $\mathrm{A}-2$ ), and each of his allowed good local responses has a strictly positive probability to be selected (assumption A-3)."

The dynamics proposed by Bala and Goyal (2000a) is based on global instead of local actions. Observe that each good local response is also a good global response. So, if we let agents play good global responses, where each good global response has a positive probability to be selected, then our procedure always reaches a local-Nash network that is also global-Nash. When we restrict agents to play neutral global responses only if they are neutral local actions that satisfy $A-3 \mathrm{a}$ to $\mathrm{A}-3 \mathrm{c}$, then it is easily verified that our procedure also terminates.

However, it is not known whether our procedure also terminates if agents have to play best global responses. This would be interesting to examine. 


\section{Chapter 6}

\section{Payoff properties in the one-way flow model}

In this chapter, we examine the framework of payoff properties that is used in Chapters 4 and 5 , and relate it to the one-way flow model studied in Chapter 2.

In section 4.6 we already showed that B\&G-1 functions with owner-homogeneous, non-negative link costs and heterogeneous, non-negative profits satisfy all properties, and therefore imply the existence of local- and global-Nash networks. Furthermore, we showed that B\&G-1 functions with heterogeneous link costs, that satisfy certain triangle inequality conditions, satisfy all properties except DE. For those functions, we know that local-Nash networks exist.

In this chapter, we fully characterize the set of B\&G-1 payoff functions that satisfy all properties. Here, we consider the largest class of B\&G-1 functions as possible, i.e. with heterogeneous link costs and profits, and without non-negativity constraints. We show that the class of orderly B\&G-1 functions extends the class of B\&G-1 functions defined by the triangle inequality conditions of Theorem 4.6.3. Further, we show that in the context of B\&G-1 functions, property DE is equivalent with owner-homogeneous link costs and non-negative profits.

This chapter is outlined as follows. In Section 6.1 we provide notations. Then, in Section 6.2 we provide a full characterization of B\&G-1 functions that satisfy the properties that define orderliness. Finally, in Section 6.3 we examine property DE in the context of B\&G-1 functions.

This chapter is based on Derks and Tennekes (2007). 


\subsection{Notations}

In this chapter we denote a B\&G-1 function as

$$
\pi_{i}(g)=v_{i}\left(N_{i}(g)\right)-c_{i}\left(N_{i}^{d}(g)\right)
$$

where $v_{i}(S)=\sum_{j \in S} v_{i j}$ and $c_{i}(S)=\sum_{j \in S} c_{i j}$ are shorthand notations for the profits respectively the link costs. Profits and link costs may be negative in this chapter.

Recall from Sections 2.2 .2 and 4.6 that we may assume that $v_{i i}=0$, because the transformation $\pi_{i}^{\prime}(g)=\pi_{i}(g)-v_{i i}$ does not have influence on the strategic behavior of agent $i$. Therefore, $\pi_{i}\left(g_{-i}\right)=0$ for all networks $g$.

\subsection{Characterization}

In this section, we provide necessary and sufficient conditions for B\&G-1 functions for being orderly.

Notice that a link $(j, i)$ is beneficial if and only if $c_{i j} \leq v_{i}(S)$, where $S$ is the set of agents that $i$ observes by using $(j, i)$.

First of all, it is clear that all B\&G-1 functions satisfy DA and BS (see Lemma 4.6.1).

It is also clear that property $\mathbf{N A}$ is satisfied by $\pi_{i}$ if and only if $c_{i j} \geq 0$ for all $j \in N$. We will refer to this condition as property NNC:

NNC (short for non-negative costs) $\quad c_{i j} \geq 0 \quad$ for all $j \in N$.

Property BG is satisfied if $v_{i j} \geq 0$ for all $j \in N$. Indeed, if $g^{\prime}=g+(k, r)$, then the set of agents that agent $i$ observes in $\left(g^{\prime}\right)_{-i j}$ is a superset of the set of agents that $i$ observes in $g_{-i j}$. It follows that if all profits are non-negative, then $(i, j)$ is beneficial in $g^{\prime}$ whenever it is beneficial in $g$. However, the following example shows that the reverse does not hold, i.e. BG does not imply non-negativity of the profits.

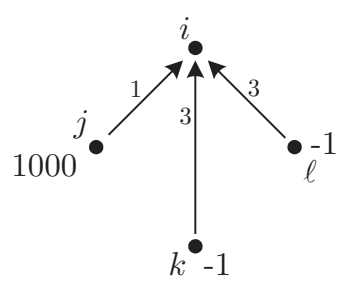

Figure 6.1: Network with some negative profits.

Example 6.2.1. Let $N=\{i, j, k, \ell\}$, and let $\pi_{i}$ be a B\&G-1 function. In Figure6.1, the numbers next to the nodes are the profits for agent $i: v_{i j}=1000$ and $v_{i k}=v_{i \ell}=-1$. The numbers next to the links are the link costs: $c_{i j}=1$ and $c_{i k}=c_{i \ell}=3$. 
Obviously, link $(j, i)$ is beneficial in any network on $N$. Links $(k, i)$ and $(\ell, i)$ are only beneficial in networks on $N$ where $j$ is observed by $k$ and $\ell$ respectively. In these networks, the beneficiality of $(k, i)$ respectively $(\ell, i)$ is guaranteed when adding other links. Therefore, we conclude that $\pi_{i}$ satisfies BG while some profits are negative. $\diamond$

The following lemma provides a full characterization of property BG with respect to B\&G-1 payoff functions.

Lemma 6.2.2. Let $\pi_{i}$ be a BEG-1 function. Then $\pi_{i}$ satisfies $\boldsymbol{B} \boldsymbol{G}$ if and only if the following property holds:

$\boldsymbol{P B G}$ If $c_{i j} \leq v_{i}(S)$ for agent set $S \subset N$ and agent $j \in S$, then $c_{i j} \leq v_{i}\left(S^{\prime}\right)$ for all $S^{\prime} \supset S$.

Proof. Suppose that PBG does not hold. Then sets $S \subset N$ and $S^{\prime} \supset S$ exist with $j \in S$ such that $c_{i j} \leq v_{i}(S)$ and $c_{i j}>v_{i}\left(S^{\prime}\right)$. Let $T=S^{\prime} \backslash S$. Consider a network $g$ where all agents in $S$ form a component with $j$ being a topagent, and all agents in $T$ form a component where agent $k$ is a topagent. Then $(j, i)$ is beneficial in $g$. However, in the network $g+(k, j)$, we have $\pi_{i}\left((g+(k, j))_{-i j}\right)=v_{i}(S)+v_{i}(T)-c_{i j}=v_{i}\left(S^{\prime}\right)-c_{i j}<0$. Therefore, $(j, i)$ is not beneficial in $g+(k, j)$. Hence $\mathbf{B G}$ is not satisfied.

Now suppose that BG is not satisfied. Then a network $g$ exists that does not contain link $(k, r)$ where $\pi_{i}\left(g_{-i j}\right) \geq 0$ and $\pi_{i}\left((g+(k, r))_{-i j}\right)<0$. Let $S$ be the set of agents that $i$ observes in $g_{-i j}$ and $S^{\prime}$ be the set of agents that $i$ observes in network $(g+(k, r))_{-i j}$. Clearly $S^{\prime} \supseteq S$. Since $\pi_{i}\left(g_{-i j}\right) \neq \pi_{i}\left((g+(k, r))_{-i j}\right)$, it follows that $S^{\prime} \neq S$ and hence that $S^{\prime} \supset S$. Since $\pi_{i}\left(g_{-i j}\right)=v_{i}(S)-c_{i j} \geq 0$ and $\pi_{i}\left((g+(k, r))_{-i j}\right)=v_{i}\left(S^{\prime}\right)-c_{i j}<0$, it follows that PBG does not hold.

For the remaining properties $\mathbf{B T}$ and $\mathbf{B F}$ we have the following results.

Lemma 6.2.3. Let $\pi_{i}$ be a BEG-1 function. Then $\pi_{i}$ satisfies $\boldsymbol{B} \boldsymbol{T}$ if and only if the following property holds:

$\boldsymbol{P B T}$ If $c_{i j} \leq v_{i}(S)$ for agent set $S \subseteq N$ and agent $j \in S$, then $c_{i j} \geq c_{i k}-v_{i}(T)$ for all $T \subseteq N \backslash S, k \in T$.

Proof. Suppose that PBT does not hold. Then sets $S \subseteq N$ and $T \subseteq N \backslash S$ exist with $j \in S$ and $k \in T$ such that $c_{i j} \leq v_{i}(S)$ and $c_{i j}<c_{i k}-v_{i}(T)$. Consider the network depicted in Figure 6.2, where $j$ observes $S$, and where $k$ is a unique topagent. Since $c_{i j} \leq v_{i}(S)$, link $(j, i)$ is beneficial in this network, i.e. $\pi_{i}\left(g_{-i j}\right)=v(S)-c_{i j} \geq 0$. Furthermore, we have

$$
\begin{aligned}
\pi_{i}\left(g_{-i j}\right) & =v(S)-c_{i j} \\
& >v(S)-c_{i k}+v_{i}(T) \\
& =v(S \cup T)-c_{i k} \\
& =\pi_{i}\left(g_{-i k}\right) .
\end{aligned}
$$


Since $k$ is the unique topagent in $g_{-i}^{j}$, we have shown that $\mathbf{B T}$ is not satisfied.

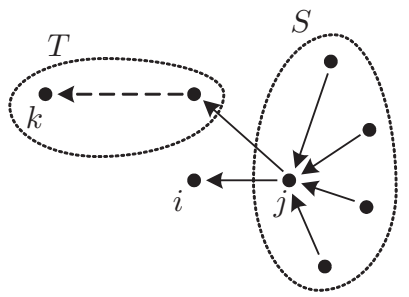

Figure 6.2: Situation where $k$ is the unique topagent in $g_{-i}^{j}$.

Now suppose that BT is not satisfied. Then a network $g$ exists where $(j, i)$ is beneficial and where a topagent exists in $g_{-i}^{j}$ such that for each topagent $k$ in $g_{-i}^{j}$, we have $\pi_{i}\left(g_{-i k}\right)<\pi_{i}\left(g_{-i j}\right)$. Let $S=N_{i}\left(g_{-i j}\right)$ be the set of agents that $i$ observes via $(j, i)$. Since $(j, i)$ is beneficial, we have $c_{i j} \leq v_{i}(S)$. Let $T$ be the set of all other agents in component $g_{-i}^{j}$. Clearly, $T \neq \emptyset$, because otherwise $j$ is a topagent and hence $\mathbf{B T}$ is satisfied. Take a topagent $k \in T$. Clearly $S \cup T=N_{i}\left(g_{-i k}\right)$. We have

$$
\begin{aligned}
& 0>\pi_{i}\left(g_{-i k}\right) \quad-\pi_{i}\left(g_{-i j}\right) \\
& =v_{i}(S \cup T)-c_{i k}-\left(v_{i}(S)-c_{i j}\right) \\
& =v_{i}(T)-c_{i k}+c_{i j} \text {. }
\end{aligned}
$$

Hence, it follows that $c_{i j}<c_{i k}-v_{i}(T)$. Therefore PBT does not hold.

Lemma 6.2.4. Let $\pi_{i}$ be a $B \mathscr{E} G-1$ function. Then $\pi_{i}$ satisfies $\boldsymbol{B F}$ if and only if the following property holds:

$\boldsymbol{P B F}$ If $c_{i j} \leq v_{i}(S)$ for agent set $S \subseteq N$ and agent $j \in S$, then $c_{i k} \leq v_{i}\left(S^{\prime}\right)$ for all $S^{\prime} \supseteq S, k \in S^{\prime} \backslash\{j\}$

Proof. Suppose that PBF does not hold. Then sets $S$ and $S^{\prime} \supseteq S$ and agents $j \in S$ and $k \in S^{\prime} \backslash\{j\}$ exist where $c_{i j} \leq v_{i}(S)$ and where $c_{i k}>v_{i}\left(S^{\prime}\right)$. Consider a network $g$ where $i$ observes all agents in $S$ in $g_{-i j}$, where $g_{-i}^{j}$ is proper, and where $(i, j) \in g$. Further, let $k$ be a topagent in $g_{-i}^{j}$ who is farthest away from $i$. Let $i$ observe all agents in $S^{\prime}$ in $g_{-i k}$. Link $(j, i)$ is beneficial because $c_{i j} \leq v_{i}(S)$ and link $(k, i)$ is not beneficial because $c_{i k}>v_{i}\left(S^{\prime}\right)$. Therefore, $\mathbf{B F}$ is not satisfied.

Now suppose that $\mathbf{B F}$ is not satisfied. Then a network $g$ exists that contains a proper component $g_{-i}^{j}$ where $i$ is active, link $(j, i)$ is beneficial, $k$ is the farthest away from $i$, and where link $(k, i)$ is not beneficial. Let $S^{\prime}$ be the set of agents in $g_{-i}^{j}$ and let $S \subseteq S^{\prime}$ be the set of agents that $j$ observes in this component. Since $(j, i)$ is beneficial it follows that $c_{i j} \leq v_{i}(S)$. Agent $k$ is a topagent, because this component is proper. Therefore $\pi_{i}\left(g_{-i k}\right)=v_{i}\left(S^{\prime}\right)-c_{i k}$. Since $(k, i)$ is not beneficial, it follows that $c_{i k}>v_{i}\left(S^{\prime}\right)$. Therefore, property PBF does not hold. 
Observe that properties PBG and PBF exhibit similarities. In the following theorem we show that $\mathbf{P B F}$ implies $\mathbf{P B G}$.

Lemma 6.2.5. In the class of BEG-1 functions, property $\mathbf{P B F}$ implies $\boldsymbol{P B G}$.

Proof. Let $\pi_{i}$ be a B\&G-1 function that satisfies PBF. Let $S \subseteq N$ with $j \in S$. If $c_{i j} \leq v_{i}(S)$ then according to $\mathbf{P B F}$ we have $c_{i k} \leq v_{i}\left(S^{\prime}\right)$ for each superset $S^{\prime} \supseteq S$ and each agent $k \in S^{\prime} \backslash S$. By applying $\mathbf{P B F}$ to the latter inequality, with the roles of $j$ and $k$ interchanged, we obtain $c_{i j} \leq v_{i}\left(S^{\prime}\right)$. Therefore we conclude that property PBG holds.

To summarize our results thus far, we know that properties DA and BS are satisfied by all B\&G-1 functions, property NA requires that link costs are non-negative (NNC), and we know that PBF implies PBG. Therefore we obtain the following theorem.

Theorem 6.2.6. Let $\pi_{i}$ be a BEGG-1 function. Then $\pi_{i}$ is orderly if and only if

$$
(\boldsymbol{N N C}) \quad c_{i j} \geq 0 \quad \forall j \in N, \text { and }
$$

if $c_{i j} \leq v_{i}(S)$ for agent set $S \subseteq N$ and agent $j \in S$ then

$$
\begin{array}{lll}
(\boldsymbol{P B T}) & c_{i j} \geq c_{i k}-v_{i}(T) & \forall T \subseteq N \backslash S, k \in T, \text { and } \\
(\boldsymbol{P B F}) & c_{i k} \leq v_{i}\left(S^{\prime}\right) & \forall S^{\prime} \supseteq S, k \in S^{\prime} \backslash\{j\}
\end{array}
$$

By Theorem 4.3.1, where the existence of local-Nash networks for orderly joint payoff functions is proved, we obtain the following result.

Corollary 6.2.7. For joint BEG-1 functions that satisfy $\boldsymbol{N} \boldsymbol{N} \boldsymbol{C}, \boldsymbol{P B} \boldsymbol{T}$, and $\boldsymbol{P B F}$, local-Nash networks exist.

Observe that these conditions are weaker (i.e. they are satisfied by more B\&G-1 functions) than the triangle conditions provided in Theorem 4.6.3. For instance, when $c_{i j}=3$ and $v_{i j}=1$ for all $i, j \in N$, then (4.17) is not satisfied, whereas NNC, PBT, and $\mathbf{P B F}$ are.

It can easily be verified that the converse of Theorem 6.2.6 is not true, i.e. examples exist of joint B\&G-1 functions that do not satisfy NNC, PBT, and PBF while localNash networks exist.

In Figure 6.3 we show the characterization of B\&G-1 functions graphically. Here, we draw four cycles which correspond to the properties PBT, NNC, PBF, and PBG. The intersections of these cycles create twelve area's, labeled from 1 to 12 . Vertices inside a cycle correspond to B\&G-1 functions that satisfy the corresponding property and vertices outside the cycle correspond to B\&G-1 functions that do not satisfy the corresponding property. For instance, all vertices in the area 7 are contained inside the 
cycle of PBT, and outside the other cycles. Therefore, the payoff functions in that area satisfy PBT, and none of the other properties.

The areas are listed in Table 6.1. Per area, we show which properties are satisfied and we provide an example that proves non-emptyness of that area. All these examples are three-person games. For area's 9 and 12 we use two examples each, to which we will refer in the next section.

The gray area (number 12) in Figure 6.3 is the area that corresponds to B\&G-1 functions that are orderly. For an example of an orderly B\&G-1 function, we used a trivial one (12a) with all $c$ and $v$ values equal to 0 . Example $12 \mathrm{~b}$ shows that B\&G-1 functions with heterogeneity among agents and link costs can be orderly as well.

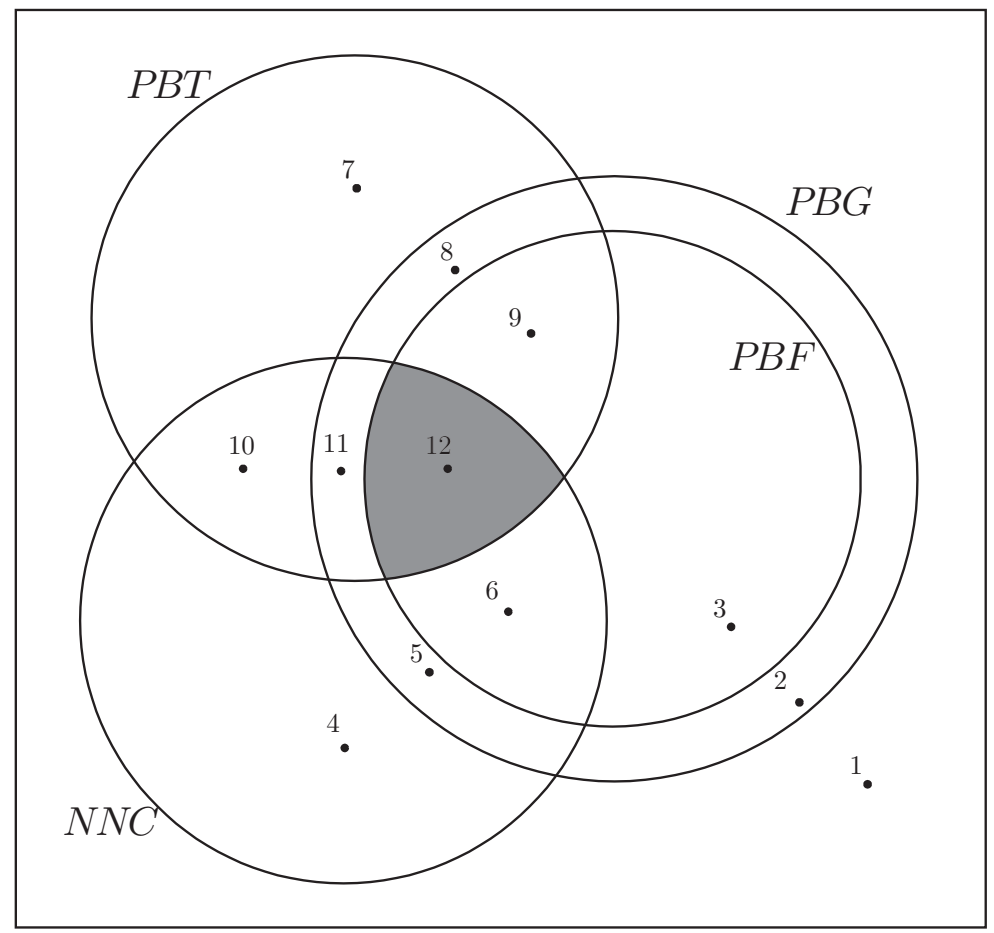

Figure 6.3: The characterization of B\&G-1 functions. The gray area corresponds to the class of orderly B\&G-1 functions. 


\begin{tabular}{lllllrrrr}
\hline Area & \multicolumn{2}{l}{ Properties } & \multicolumn{7}{c}{ Example } \\
& PBT & NNC & PBF & PBG & $c_{i j}$ & $c_{i k}$ & $v_{i j}$ & $v_{i k}$ \\
\hline 1 & $\mathrm{n}$ & $\mathrm{n}$ & $\mathrm{n}$ & $\mathrm{n}$ & -1 & 0 & 0 & -2 \\
2 & $\mathrm{n}$ & $\mathrm{n}$ & $\mathrm{n}$ & $\mathrm{y}$ & -1 & 1 & 0 & -1 \\
3 & $\mathrm{n}$ & $\mathrm{n}$ & $\mathrm{y}$ & $\mathrm{y}$ & -1 & 3 & 2 & 1 \\
4 & $\mathrm{n}$ & $\mathrm{y}$ & $\mathrm{n}$ & $\mathrm{n}$ & 0 & 2 & 0 & -1 \\
5 & $\mathrm{n}$ & $\mathrm{y}$ & $\mathrm{n}$ & $\mathrm{y}$ & 0 & 2 & 0 & 1 \\
6 & $\mathrm{n}$ & $\mathrm{y}$ & $\mathrm{y}$ & $\mathrm{y}$ & 1 & 3 & 2 & 1 \\
7 & $\mathrm{y}$ & $\mathrm{n}$ & $\mathrm{n}$ & $\mathrm{n}$ & 1 & -2 & 3 & -3 \\
8 & $\mathrm{y}$ & $\mathrm{n}$ & $\mathrm{n}$ & $\mathrm{y}$ & -1 & 3 & -2 & 2 \\
$9 \mathrm{a}$ & $\mathrm{y}$ & $\mathrm{n}$ & $\mathrm{y}$ & $\mathrm{y}$ & -1 & -1 & 3 & 3 \\
$9 \mathrm{~b}$ & $\mathrm{y}$ & $\mathrm{n}$ & $\mathrm{y}$ & $\mathrm{y}$ & 1 & -1 & 3 & 1 \\
10 & $\mathrm{y}$ & $\mathrm{y}$ & $\mathrm{n}$ & $\mathrm{n}$ & 3 & 0 & 4 & -2 \\
11 & $\mathrm{y}$ & $\mathrm{y}$ & $\mathrm{n}$ & $\mathrm{y}$ & 2 & 3 & 1 & 1 \\
$12 \mathrm{a}$ & $\mathrm{y}$ & $\mathrm{y}$ & $\mathrm{y}$ & $\mathrm{y}$ & 0 & 0 & 0 & 0 \\
$12 \mathrm{~b}$ & $\mathrm{y}$ & $\mathrm{y}$ & $\mathrm{y}$ & $\mathrm{y}$ & 1 & 2 & 4 & 1 \\
\hline
\end{tabular}

Table 6.1: The twelve area's in Figure 6.3.

\subsection{Downstream efficiency}

In this section, we study the role of property downstream efficiency (DE) with respect to B\&G-1 functions. In the next lemma, we show that DE is equivalent to ownerhomogeneous link costs and non-negative profits.

Lemma 6.3.1. Let $\pi_{i}$ be a B\&G-1 function. Then $\pi_{i}$ satisfies $\boldsymbol{D E}$ if and only if

$$
\begin{array}{llr}
\boldsymbol{O H C} \text { (short for owner-homogeneous costs) } & c_{i j}=c_{i k} & \forall j, k \in N, \\
\boldsymbol{N N P} \text { (short for non-negative profits) } & v_{i j} \geq 0 & \forall j \in N .
\end{array}
$$

Proof.

(DE implies OHC) Suppose that DE is satisfied, and let $j$ and $k$ be agents in $N \backslash\{i\}$. Consider a network $g$, where all agents in $N \backslash\{i\}$ are contained in a directed cycle and where $i$ is isolated. Since there exists a directed path between $j$ and $k$ in both ways, we have by DE that $\pi_{i}(g+(j, i))=\pi_{i}(g+(k, i))$. Therefore it follows that $c_{i j}=c_{i k}$ for all $j, k \in N$.

(DE implies NNP) Suppose that DE is satisfied. Let $j$ and $k$ be agents in $N \backslash\{i\}$. Consider network $g=\{(k, j),(k, i)\}$. Then by $\mathbf{D E}$ we have

$$
0 \leq \pi_{i}(g+(j, i)-(k, i))-\pi_{i}(g)=c_{i k}-c_{i j}+v_{i j}
$$

Since DE implies OHC, it follows that $v_{i j} \geq 0$.

(OHC and NNP imply DE) Suppose that OHC and NNP are satisfied and DE is not. Then a network $g$ exists where $\pi_{i}(g+(j, i))<\pi_{i}(g+(k, i))$, where $(j, i)$ 
and $(k, i)$ do not exist, and where a directed path from $k$ to $j$ exists in $g_{-i}$. Since link costs are owner-homogeneous (by OHC), there is no path vice versa, for otherwise $\pi_{i}(g+(j, i))=\pi_{i}(g+(k, i))$. Let $S \subset N$ be the set of agents that $i$ observes in network $g+(j, i)$ and not in $g+(k, i)$. Notice that $S \neq \emptyset$, since there is no directed path from $j$ to $k$ in $g_{-i}$, i.e. $j \in S$. We have

$$
0>\pi_{i}(g+(j, i))-\pi_{i}(g+(k, i))=v_{i}(S)-c_{i j}+c_{i k}
$$

Because $\mathbf{O H C}$ is satisfied, it follows that $v_{i}(S)<0$. Hence an agent $\ell$ exists in $S$ such that $v_{i \ell}<0$. This contradicts NNP.

We obtain the following theorem.

Theorem 6.3.2. Let $\pi_{i}$ be a B\&G-1 function. Then $\pi_{i}$ is orderly and satisfies $\boldsymbol{D E}$ if and only if

$$
\begin{array}{lll}
(\boldsymbol{N N C}) & c_{i j} \geq 0 & \forall j \in N, \\
(\boldsymbol{O H C}) & c_{i j}=c_{i k} & \forall j, k \in N, \\
(\boldsymbol{N N P}) & v_{i j} \geq 0 & \forall j \in N .
\end{array}
$$

From Corollay 4.3.2 we obtain the following result which is also proved in Theorems 2.2 .2 and 2.2 .8 .

Corollary 6.3.3. For joint BEG-1 functions with owner-homogeneous and non-negative link costs and heterogeneous and non-negative profits, global-Nash networks exist.

In the next proposition, we show that $\mathbf{D E}$ implies $\mathbf{B T}$ and $\mathbf{B F}$ in the context of the one-way flow model. In other words, we show that owner-homogeneous link costs (OHC) and non-negative profits (NNP) imply PBT and PBF.

Proposition 6.3.4. The properties $\boldsymbol{O H C}$ and $\mathbf{N N P}$ imply $\boldsymbol{P B T}$ and $\boldsymbol{P B F}$.

Proof. Let $\pi_{i}$ be a payoff function that satisfies $\mathbf{O H C}$ and NNP.

By NNP we have that $v_{i}(T) \geq 0$ for all $T \subseteq N$. Furthermore, by $\mathbf{O H C}, c_{i j}=c_{i j}$ for all $j, k \in N$. Therefore if follows that $c_{i j} \geq c_{i k}-v_{i}(T)$ and hence that PBT is satisfied.

Let $S \subseteq N$ be a set of agents with $j \in S$ such that $c_{i j} \leq v_{i}(S)$. Let $S^{\prime} \supset S$. By NNP it follows that $c_{i j} \leq v_{i}\left(S^{\prime}\right)$. By $\mathbf{O H C}$ it follows that $c_{i k}=c_{i j} \leq v_{i}\left(S^{\prime}\right)$, for all $k \in S^{\prime} \backslash\{j\}$. Therefore, $\mathbf{P B F}$ is satisfied.

Examples 9a, 9b, 12a, and 12b show that neither NNC implies $\mathbf{O H C}$ and NNP nor vice versa. Figure 6.4 provides a graphical characterization of $\mathbf{O H C}$ and $\mathbf{N N P}$ related to the other properties. 


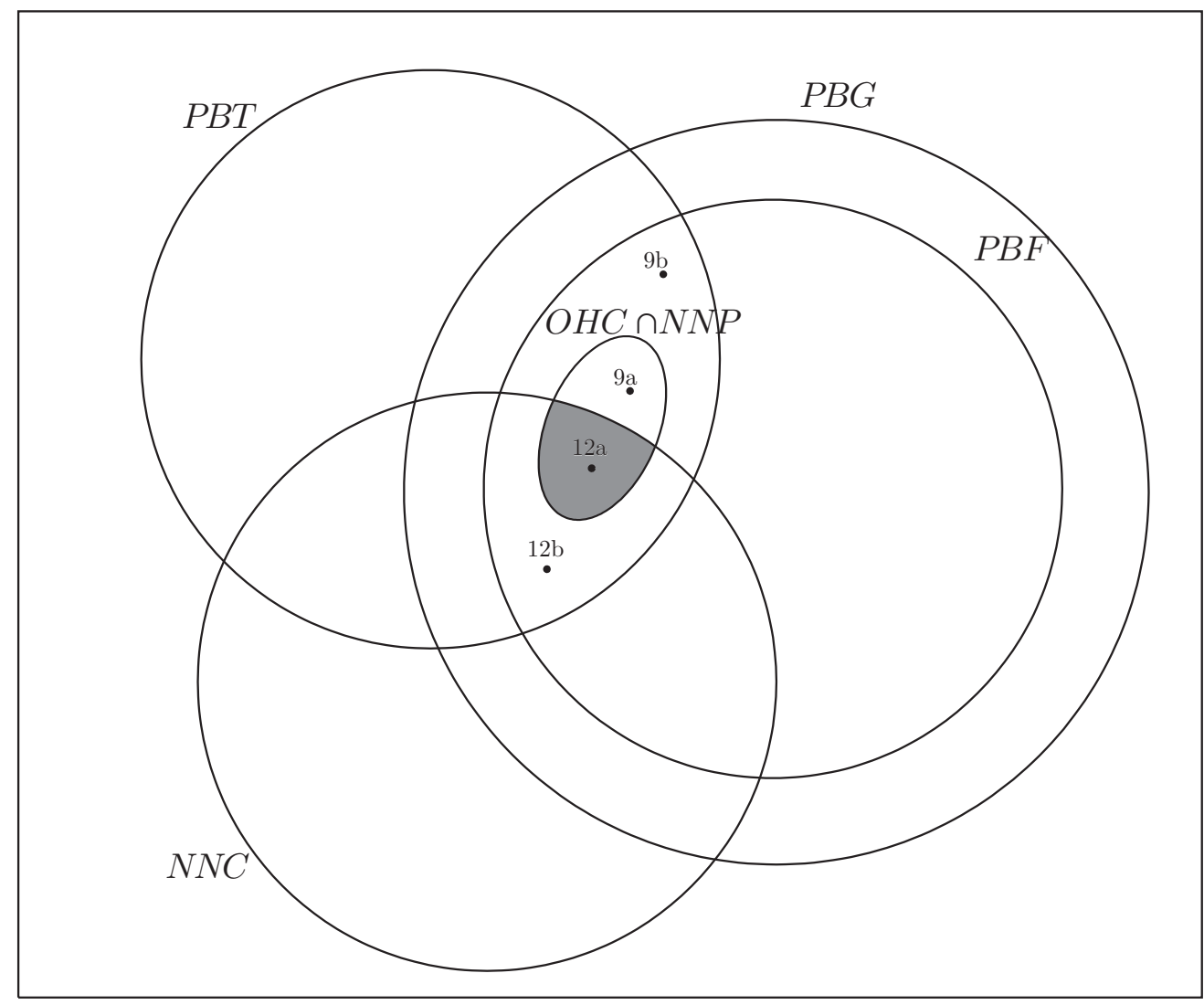

Figure 6.4: The characterization of B\&G-1 functions. The gray area corresponds to the class of orderly B\&G-1 functions that also satisfy DE. 



\section{Chapter 7}

\section{An axiomatic approach covering the two-way flow model}

In this chapter we generalize the two-way flow model of network formation, which already has been treated in Section 3.6. The only difference with the one-way flow model, is that profits are distributed in the network in both directions of the links. The links are still directed in the two-way flow model, in order to indicate the owners. We provide an axiomatic framework of payoff properties that covers this model. We compare the properties that we propose to the properties that are used in Chapter 4 to cover the one-way flow model.

In the previous chapters, the links are depicted as arcs pointing at their owners. In the one-way flow model, the direction of the arcs also indicates the information flow. Consider the network depicted in Figure 7.1(a). In the one-way flow model, agent 1 does not receive profits (e.g. information) from anyone, and agents 2 and 3 only receive profits from agent 1 . However, in the two-way flow model, all three agents share profits. Therefore, we prefer to depict a link owned by agent $i$ as a line that is cut by a short line next to $i$ (see Figure 7.1(b)). Thus, in the two-way flow model, profits flow in both directions of the links, and the owners of the links are indicated by short lines.

The two-way flow model is introduced by Bala and Goyal (2000a). They prove the existence of Nash networks and characterize the architecture of those networks for payoff functions with homogeneous link costs and profits. Galeotti et al. (2006) extend this model by introducing heterogeneous link costs and profits. They characterize the architecture of strict Nash networks, while assuming such networks exist. Haller et al. (2007) prove the existence of Nash networks for payoff functions with heterogeneous profits and homogeneous link costs. Furthermore, they show by means of a counterexample that Nash networks do not always exist for games with heterogeneous link costs. 


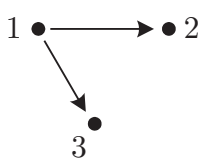

(a)

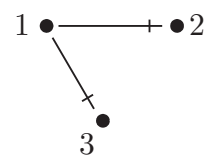

(b)

Figure 7.1: Representations of the same network for the one-way (a) and the two-way (b) flow model.

However, for a subclass of those games, Kamphorst and Van der Laan (2007) prove the existence of Nash networks. Here, agents are divided into groups which are located on a line. The cost of a link is increasing in the distance between the groups where both adjacent agents belong to. When both agents belong to the same group, this cost is a fixed positive value.

In Chapter 4, we proposed a framework of axiomatic payoff properties that is inspired by the one-way flow model. A full characterization of B\&G-1 functions (i.e. payoff functions used in that model) that satisfy our properties has been provided in Chapter 6. We showed that all B\&G-1 functions with heterogeneous profits and ownerhomogeneous link costs satisfy these properties, as well as a subset of B\&G-1 functions with heterogeneous profits and link costs.

However, the axiomatic framework in Chapter 4 does not cover B\&G-2 functions, i.e. payoff functions used in the two-way flow model. In particular, property $\mathbf{B G}$ is violated. According to this property, each link remains beneficial when another link is added to the network. To see why property $\mathbf{B G}$ is violated, consider a component where agent $i$ is active by an own link. Via this link, agent $i$ observes the whole component (recall that profits flow in both directions of the links in the two-way flow model). Suppose that another agent in that component, say $j$, forms link $(i, j)$. Although this action by $j$ does not make sense, it is allowed. Then $i$ 's own link becomes redundant, since he now observes the whole component by link $(i, j)$. If his own link has a strictly positive cost, then it is non-beneficial in the obtained network. This violates property BG.

In this chapter we develop axiomatic payoff properties that are intuitive in the context of the two-way flow model. With these payoff properties, we prove the existence of local- and global-Nash networks. We do this by generalizing the short and elementary proof by Haller et al. (2007). Although they did not use the definitions of local actions and local-Nash networks, their line of proof is based on local improvements, which will be highlighted in our approach.

We provide a full characterization of B\&G-2 functions that satisfy our properties. We show that B\&G-2 functions with negative profits exist that satisfy these properties, and therefore imply the existence of local- and global-Nash networks. Further, we show that these properties are satisfied by B\&G-2 functions where a subset of links are 
affordable and owner-homogeneous while the other links are too expensive in any case.

Furthermore, we provide examples of payoff functions that do not belong to the two-way flow model, even though they satisfy all our properties. These functions also take other properties of the network architecture into account, for instance the set of agents contained in a cycle. Therefore, our approach not only covers the two-way flow model with owner-homogeneous links costs, but also generalizes it.

This chapter is outlined as follows. First, we present the model and the notations that we will use throughout. Then, in Section 7.2 we propose a framework of axiomatic payoff properties that is based on the two-way flow model. For payoff functions that satisfy these properties, we prove the existence of local- and global-Nash networks. In Section 7.3 we show that the properties are independent of each other. In Section 7.4 we provide a full characterization of B\&G-2 functions that satisfy all properties, and we give examples of non-B\&G-2 functions that satisfy all properties. Finally, in Section 7.5 we provide concluding remarks.

This chapter is based on Derks et al. (2009a).

\subsection{Model and notations}

In this chapter, we will use the same notations as in the previous chapters.

Recall that

$$
N_{i}^{d}(g)=\{j \in N:(j, i) \in g\} .
$$

Further, recall the following definitions from Section 3.6, where we studied the complexity of finding best responses in the two-way flow model. Let

$$
N_{i}^{u}(g)=\{j \in N: \text { an undirected path between } j \text { and } i \text { exists in } g\} .
$$

In the context of the two-way flow model, we say that agent $i$ observes agent $j$ in network $g$ if $j \in N_{i}^{u}(g)$. Payoff function $\pi_{i}(g)$ is called a B\&G-2 function if

$$
\pi_{i}(g)=v_{i}\left(N_{i}^{u}(g)\right)-c_{i}\left(N_{i}^{d}(g)\right)
$$

where we use the following shorthand notation: $v_{i}(S)=\sum_{j \in S} v_{i j}$ and $c_{i}(S)=\sum_{j \in S} c_{i j}$. Here, $v_{i j}$ is the profit that $i$ receives from observing $j$ and $c_{i j}$ is the cost of link $(j, i)$.

In Chapters 2 and 4, we saw that proper networks (networks in which each agent has at most one outgoing link) are essential in the one-way flow model. However, proper networks are not suitable candidates for Nash networks in the two-way flow model. For instance, consider a proper network with a cycle. Since profits flow in both directions of the arcs, one link in this cycle is redundant. Therefore, this cannot be a Nash network, unless all links in this cycle are for free.

A more appropriate architectural property is the following. Let network $g$ be called minimal (for the undirected case) if for each link $(j, i)$, an undirected path between $j$ and $i$ does not exist in $g-(j, i)$. An example of a minimal network is depicted in 
Figure 7.2. In the literature, minimal networks are essential in the two-way flow model. For instance, Galeotti et al. (2006) show that all strict Nash networks are minimal.

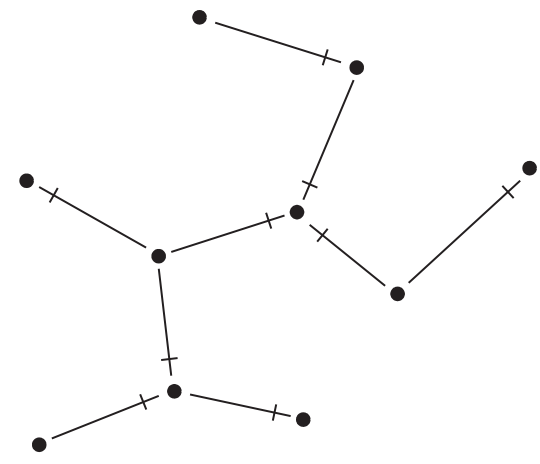

Figure 7.2: A minimal network.

\subsection{Axiomatization}

In this section, we develop intuitive axiomatic payoff properties for the two-way flow model, in such away that we are able to prove the existence of Nash networks. For this purpose, we will use the constructive proof provided by Haller et al. (2007). This proof shows that Nash networks exist for games with homogeneous link costs and heterogeneous profits. We develop axiomatic payoff properties such that this proof holds for a more general class of payoff functions.

The first property that we introduce, disjoint additivity, is also used in our framework in Chapter 4.

Property DA We say that a payoff function $\pi_{i}$ is disjoint additive (DA for short), if for each two networks $g$ and $g^{\prime}$, disjoint w.r.t. an agent $i$, we have

$$
\pi_{i}\left(g+g^{\prime}\right)=\pi_{i}(g)+\pi_{i}\left(g^{\prime}\right) .
$$

Recall the following definitions from Chapter 4 . Link $(j, i)$ is called profitable in $g$ if:

$$
\pi_{i}(g) \geq \pi_{i}(g-(j, i)) \quad \text { if }(j, i) \in g,
$$

and

$$
\pi_{i}(g+(j, i)) \geq \pi_{i}(g) \text { if }(j, i) \notin g .
$$

Further, link $(j, i)$ is called beneficial in network $g$ whenever

$$
\pi_{i}\left(g_{-i j}\right) \geq \pi_{i}\left(g_{-i}^{j}\right)
$$


Thus, the influence of a single link $(j, i)$ is measured in component $g_{-i}^{j}$. A network is called beneficial if all existing links in it are beneficial.

In the following result, we compare profitability and beneficiality with respect to minimal networks.

Lemma 7.2.1. If network $g$ is minimal, and $\pi$ is disjoint additive, then profitability and beneficiality are equivalent notions for all existing links and for some non-existing links:

(i) $\quad \pi_{i}(g)-\pi_{i}(g-(j, i))=\pi_{i}\left(g_{-i j}\right)-\pi_{i}\left(g_{-i}^{j}\right) \quad$ for all $(j, i) \in g$,

(ii) $\quad \pi_{i}(g+(j, i))-\pi_{i}(g)=\pi_{i}\left(g_{-i j}\right)-\pi_{i}\left(g_{-i}^{j}\right) \quad$ for all $(j, i) \notin g$ with $j \notin N_{i}^{u}(g)$.

Proof. First, let $(j, i) \in g$. Since $g$ is minimal, $g_{-i j}$ and $g-g_{-i j}$ are $i$-disjoint. Therefore, by DA we have

$$
\pi_{i}(g)=\pi_{i}\left(g_{-i j}\right)+\pi_{i}\left(g-g_{-i j}\right)
$$

Since $g_{-i j}$ and $g-g_{-i j}$ are $i$-disjoint, networks $g_{-i}^{j}$ and $g-g_{-i}^{j}$ are also $i$-disjoint. Hence by DA we obtain

$$
\pi_{i}(g-(j, i))=\pi_{i}\left(g_{-i}^{j}\right)+\pi_{i}\left(g-g_{-i}^{j}-(j, i)\right) .
$$

With $g-g_{-i j}=g-g_{-i}^{j}-(j, i)$, we obtain (i) from 7.2 and 7.3 .

Now, let $(j, i) \notin g$, with $j \notin N_{i}^{u}(g)$. Let $g^{\prime}=g+(j, i)$. Since $g$ is minimal and $j \notin N_{i}^{u}(g)$, it follows that $g^{\prime}$ is also minimal. Since $(j, i) \in g^{\prime}$, (i) can be applied with respect to $g^{\prime}$. Hence, we obtain (ii).

The next property is proposed in Chapter 4 .

Property NA We say that $\pi_{i}$ satisfies NA (naturality) if $\pi_{i}(g+(k, i)) \leq \pi_{i}(g)$ whenever there is a directed path from $k$ to $i$ in network $g$.

According to this property, for each agent $k \in N_{i}(g)$, agent $i$ 's payoff will not increase after adding $(k, i)$ to $g$. This property is intuitive in the one-way flow case, because when $i$ already observes $k$ (see Figure 7.3 ), then he will not be better off by forming link $(k, i)$. However, in the two-way flow case, agent $i$ observes $k$ already if an undirected path exists between them (see Figure $7.3 \mathrm{~b}$ ). Therefore we propose the following alternative.

Property $\mathbf{N A}_{\mathbf{u}}$ We say that $\pi_{i}$ satisfies $\mathbf{N} \mathbf{A}_{\mathbf{u}}$ (naturality, for the undirected case) if $\pi_{i}(g+(k, i)) \leq \pi_{i}(g)$ whenever there is a undirected path between $k$ and $i$ in network $g$. 


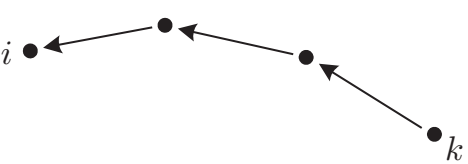

(a)

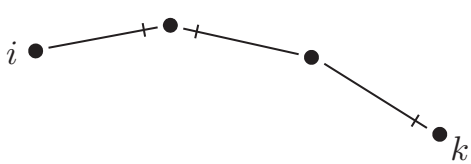

(b)

Figure 7.3: Agent $i$ observes $k$ in the one-way flow case (a) and in the two-way flow case (b).

Observe that by this property, minimal networks are preferred over non-minimal networks. More specifically, any non-minimal network $g$ can be turned into a minimal network $g^{\prime}$ by a sequence of deletions of redundant links, which are good local responses by $\mathbf{N A}_{\mathbf{u}}$.

In the following theorem we show that for payoff functions that satisfy DA and $\mathbf{N A}_{\mathbf{u}}$, each minimal local-Nash network is also a global-Nash network.

Theorem 7.2.2. Let $\pi$ be a joint payoff function that satisfies $\boldsymbol{D A}$ and $\boldsymbol{N A}_{\boldsymbol{u}}$. Then each minimal local-Nash network is global-Nash.

Proof. Let $g$ be a minimal local-Nash network. Suppose to the contrary that $g$ is not global-Nash, say $i$ can strictly improve in $g$. Let $S=N_{i}^{d}(g)$ be his current action, and let $\tilde{S}$ be a strictly improving action, such that $|\tilde{S} \backslash S|$ is as small as possible and such that among those, $|S \backslash \tilde{S}|$ is as small as possible. Let $\tilde{g}$ be the network obtained after $i$ plays $\tilde{S}$.

First we show that $\tilde{g}$ is minimal. Suppose otherwise. Then an undirected cycle exists in $\tilde{g}$. It contains $i$, since only links adjacent to $i$ have changed compared to $g$. Moreover, this cycle contains at least one link $(j, i)$, with $j \in \tilde{S} \backslash S$ (otherwise this cycle also exists in $g)$. By $\mathbf{N A}_{\mathbf{u}}$ we have $\pi_{i}(\tilde{g}) \leq \pi_{i}(g-(j, i))$, and hence the action $\tilde{S} \backslash\{j\}$ is at least as good as $\tilde{S}$. This contradicts the minimality of $|\tilde{S} \backslash S|$. Therefore, $\tilde{g}$ is minimal.

First assume $j \in \tilde{S} \backslash S$.

Suppose that $j \in N_{i}^{u}(g)$. Then an agent $k \in S$ exists such that $k \in \operatorname{Car}\left(g_{-i}^{j}\right)$. Since $g$ is local-Nash, agent $i$ does not improve his payoff by replacing $(k, i)$ with $(j, i)$, i.e.

$$
\begin{aligned}
0 & \geq \pi_{i}(g-(k, i)+(j, i)) & & -\pi_{i}(g) \\
& =\pi_{i}(g-(k, i)+(j, i))-\pi_{i}(g-(k, i)) & & -\left(\pi_{i}(g)-\pi_{i}(g-(k, i))\right) \\
& =\pi_{i}\left(g_{-i j}\right)-\pi_{i}\left(g_{-i}^{j}\right) & & -\left(\pi_{i}\left(g_{-i k}\right)-\pi_{i}\left(g_{-i}^{k}\right)\right) \\
& =\pi_{i}(\tilde{g})-\pi_{i}(\tilde{g}-(j, i)) & & -\left(\pi_{i}(\tilde{g}-(j, i)+(k, i))-\pi_{i}(\tilde{g}-(j, i))\right) \\
& =\pi_{i}(\tilde{g}) & & -\pi_{i}(\tilde{g}-(j, i)+(k, i)) .
\end{aligned}
$$


The second equality follows by applying Lemma 7.2.1(ii) to network $g-(k, i)$ and link $(j, i) \notin(g-(k, i))$ with $j \notin N_{i}^{u}(g-(k, i))$, and by applying Lemma 7.2.1(i) to network $g$ and link $(k, i) \in g$. The third equality follows by applying Lemma 7.2.1(i) to network $\tilde{g}$ and link $(j, i) \in \tilde{g}$, and by applying Lemma 7.2.1 (ii) to network $\tilde{g}-(j, i)$ and link $(k, i) \notin(\tilde{g}-(j, i))$. Notice that $k \notin N_{i}^{u}(\tilde{g}-(j, i))$, because $\tilde{g}$ is minimal, and $j$ and $k$ are active in the same component in $g_{-i}$. Hence the action $\tilde{S} \backslash\{j\} \cup\{k\}$ is at least as good as $\tilde{S}$. Since $|(\tilde{S} \backslash\{j\} \cup\{k\}) \backslash S|<|\tilde{S} \backslash S|$, we obtain a contradiction.

Hence $j \notin N_{i}^{u}(g)$. By minimality of $\tilde{g}$, it follows that $g_{-i j}$ and $\tilde{g}-g_{-i j}$ are $i$-disjoint, and also $g_{-i}^{j}$ and $\tilde{g}-g_{-i j}$. By DA we obtain

$$
\begin{aligned}
& \pi_{i}(\tilde{g}) \quad=\pi_{i}\left(\tilde{g}-g_{-i j}\right)+\pi_{i}\left(g_{-i j}\right) \quad \text { and } \\
& \pi_{i}(\tilde{g}-(j, i))=\pi_{i}\left(\tilde{g}-g_{-i j}\right)+\pi_{i}\left(g_{-i}^{j}\right) .
\end{aligned}
$$

Since $g$ is local-Nash, we have $\pi_{i}(g+(j, i)) \leq \pi_{i}(g)$. From this observation, it follows by Lemma 7.2.1(ii) that $\pi_{i}\left(g_{-i j}\right) \leq \pi_{i}\left(g_{-i}^{j}\right)$. Hence by 7.4$)$ and 7.5 we obtain $\pi_{i}(\tilde{g}) \leq \pi_{i}(\tilde{g}-(j, i))$. Hence $\tilde{S} \backslash\{j\}$ is at least as good as $\tilde{S}$, with $|(\tilde{S} \backslash\{j\}) \backslash S|<|\tilde{S} \backslash S|$. This is a contradiction.

Hence we conclude that $\tilde{S} \subseteq S$.

Now assume $j \in S \backslash \tilde{S}$.

Since $g$ is minimal local-Nash, $(j, i)$ is profitable in $g$ and by Lemma 7.2.1(i) also beneficial in $g$ and hence also in $\tilde{g}$. Since $\tilde{S} \subseteq S$ and $j \notin N_{i}^{u}(g-(j, i))$, it follows that $j \notin N_{i}^{u}(\tilde{g})$. Since $\tilde{g}$ is minimal, it follows by Lemma 7.2.1(ii) that $(j, i)$ is also profitable in $\tilde{g}$. Therefore $\tilde{S} \cup\{j\}$ is at least as good as $\tilde{S}$, with $|S \backslash(\tilde{S} \cup\{j\})|<|S \backslash \tilde{S}|$. This is a contradiction.

Hence we conclude that $\tilde{S}=S$, which contradicts $\tilde{S}$ being a strict improvement. Therefore, $g$ is global-Nash.

The following property has been proposed in Chapter 4 .

Property BG Payoff function $\pi_{i}$ satisfies BG (beneficial growth) if

$$
\pi_{i}\left((g+(k, r))_{-i j}\right) \geq \pi_{i}\left((g+(k, r))_{-i}^{j}\right)
$$

for each two agents $k, r$, whenever $\pi_{i}\left(g_{-i j}\right) \geq \pi_{i}\left(g_{-i}^{j}\right)$.

However, it does not apply to all B\&G-2 functions. To see this, consider network $g=\{(j, i)\}$, and let all profits and costs be equal to 1 . Then $\pi_{i}(g)=v_{i i}+v_{i j}-c_{i j}=1$ and $\pi_{i}\left(g_{-i}\right)=v_{i i}=1$. Thus, link $(j, i)$ is beneficial in $g$. Now consider network $g^{\prime}=g+(i, j)$. Since agent $i$ also observes agent $j$ by link $(i, j)$, his own link is redundant in $g^{\prime}$. We have $\pi_{i}\left(g^{\prime}\right)=v_{i i}+v_{i j}-c_{i j}=1$ while $\pi_{i}\left(g_{-i}^{\prime}\right)=v_{i i}+v_{i j}=2$. We can fix this by the following refinement. 
Property $\mathbf{B G}_{\mathbf{u}}$ Payoff function $\pi_{i}$ satisfies $\mathbf{B G}_{\mathbf{u}}$ (beneficial growth for the undirected case) if

$$
\pi_{i}\left((g+(k, r))_{-i j}\right) \geq \pi_{i}\left((g+(k, r))_{-i}^{j}\right)
$$

for each two agents $k, r$, whenever $g+(k, r)$ is minimal and $\pi_{i}\left(g_{-i j}\right) \geq \pi_{i}\left(g_{-i}^{j}\right)$.

Thus, a link remains beneficial if another agent adds a link such that the obtained network is minimal. The intuition behind this property is that in a minimal network, at most one undirected path exists between each pair of agents. Hence, the set of agents observed by agent $i$ via link $(j, i)$ in a minimal network $g$, is also observed uniquely via $(j, i)$ in the minimal network $g+(k, r)$.

The last property that we introduce is very demanding.

Property RP Payoff function $\pi_{i}$ satisfies RP (replacement) if the following holds. Let $g$ be a minimal network where $(j, i) \in g$. If $(j, i)$ is beneficial, then $\pi_{i}\left(g_{-i k}\right) \leq \pi_{i}\left(g_{-i j}\right)$, for each agent $k \in \operatorname{Car}\left(g_{-i}^{j}\right)$.

Applying Lemma 7.2.1, this property implies that whenever a link is profitable in a minimal network, any replacement within the same component is not a strictly improving local action.

The intuition behind this property is the following. For our line of proof, our aim is to design a property such that beneficial links in a minimal network remain beneficial after a replacement. Replacements within the same component could have a negative influence on the beneficiality of other links. Consider for instance network $g$ depicted in Figure 7.4(a), and consider the replacement of $(j, i)$ by $(k, i)$. The obtained network $g^{\prime}$ is depicted in Figure 7.4(b). Now focus on link $(j, r)$. In network $g$, agent $r$ observes 5 agents via this link, whereas in network $g^{\prime}$, he only observes 2 of them. A B\&G-2 function can be chosen such that link $(j, r)$ is beneficial in $g$ but not anymore in $g^{\prime}$. By this argument, we discourage replacements inside a component with property $\mathbf{R P}$.

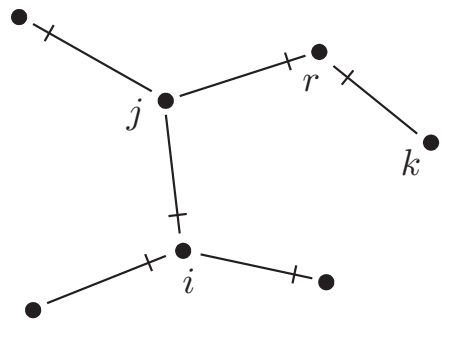

(a) Network $g$

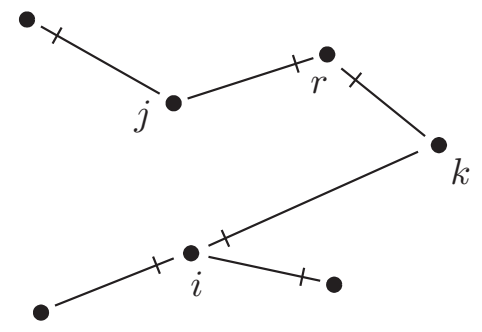

(b) Network $g^{\text {' }}$

Figure 7.4: A replacement.

Now we generalize the result of Haller et al. (2007), which is that Nash networks exist for B\&G-2 functions with heterogeneous profits and homogeneous link costs. We 
reconstruct their proof with the implementation of the payoff properties $\mathbf{D A}, \mathbf{N A}_{\mathbf{u}}$, $\mathbf{B G}_{\mathbf{u}}$, and $\mathbf{R P}$.

Theorem 7.2.3. Let $\pi$ be a joint payoff function that satisfies $\boldsymbol{D A}, \boldsymbol{N} \boldsymbol{A}_{\boldsymbol{u}}, \boldsymbol{B}_{\boldsymbol{u}}$, and $\boldsymbol{R P}$. Then a minimal local-Nash network exists.

Proof. First observe that the empty network is minimal and beneficial. Now let $g$ be any minimal and beneficial network and not local-Nash, say $i$ can improve.

By Lemma 7.2.1(i) it follows that each link in $g$ is profitable, and therefore $i$ does not gain from removing a link.

Because of $\mathbf{R P}$, agent $i$ does not strictly prefer to replace link $(k, i)$ with $(j, i)$, where $j \in \operatorname{Car}\left(g_{-i}^{k}\right)$. Now consider the replacement of $(k, i)$ with $(j, i)$, where $j \notin \operatorname{Car}\left(g_{-i}^{k}\right)$. By $\mathbf{N A}_{\mathbf{u}}$, we may assume that $j \notin N_{i}^{u}(g)$. Therefore, $g+(j, i)$ is minimal. Since $g$ is beneficial, link $(k, i)$ is beneficial in $g$, and consequently also in network $g+(j, i)$. By Lemma 7.2.1(i) it follows that $(k, i)$ is also profitable in $g+(j, i)$. Therefore, the addition of $(j, i)$ is at least as good as the replacement of $(k, i)$ by $(j, i)$.

Consequently, agent $i$ has an improving addition, say link $(j, i)$. Let the obtained network be $g^{\prime}=g+(j, i)$. By $\mathbf{N A}_{\mathbf{u}}$, we may assume that $j \notin N_{i}^{u}(g)$. Hence $g^{\prime}$ is also minimal. Since $(j, i)$ is profitable in $g^{\prime}$, it follows by Lemma 7.2.1 (i) that link $(j, i)$ is also beneficial in $g^{\prime}$. Further, the other links in $g^{\prime}$ are beneficial by $\mathbf{B G}_{\mathbf{u}}$. Hence, $g^{\prime}$ is beneficial.

Since $g^{\prime}$ is minimal and beneficial, we can repeat this step if $g^{\prime}$ is not local-Nash. At each iteration, a link is added, and therefore, the network grows. Since a minimal network of $n$ agents has at most $n-1$ links, we know that in finitely many iterations we obtain a local-Nash network.

Since the network that we obtained in this proof is minimal, we arrive at the following corollary from Theorem 7.2 .2

Corollary 7.2.4. Let $\pi$ be a joint payoff function that satisfies $\boldsymbol{D A}, \boldsymbol{N} \boldsymbol{A}_{\boldsymbol{u}}, \boldsymbol{B G}_{\boldsymbol{u}}$, and $\boldsymbol{R P}$. Then a minimal global-Nash network exists.

\subsection{Property independence}

In this section, we show independence of the four properties of our framework.

Theorem 7.3.1. The properties $\boldsymbol{D A}, \boldsymbol{N} \boldsymbol{A}_{\boldsymbol{u}}, \boldsymbol{B G}_{\boldsymbol{u}}$, and $\boldsymbol{R P}$ are independent of each other.

Proof. We show that for each property, a payoff function exists which does not satisfy that property while it satisfies all other properties.

(all but DA) The following payoff function satisfies all properties, except DA:

$$
\pi_{i}(g)=\left|N_{i}^{u}(g)\right|^{2} .
$$


Property DA is not satisfied, because for any two $i$-disjoint networks $g$ and $g^{\prime}$ where $i \in \operatorname{Car}(g) \cap \operatorname{Car}\left(g^{\prime}\right)$, we have $\left|N_{i}^{u}(g)\right|^{2}+\left|N_{i}^{u}\left(g^{\prime}\right)\right|^{2}<\left|N_{i}^{u}\left(g+g^{\prime}\right)\right|^{2}$. The properties $\mathbf{N A}_{\mathbf{u}}, \mathbf{B G}_{\mathbf{u}}$, and $\mathbf{R P}$ are clearly satisfied.

(all but $\mathbf{N} \mathbf{A}_{\mathbf{u}}$ ) The following payoff function satisfies all properties, except $\mathbf{N} \mathbf{A}_{\mathbf{u}}$ :

$$
\pi_{i}(g)=\left|N_{i}^{d}(g)\right|
$$

Property $\mathbf{N A}_{\mathbf{u}}$ is not satisfied, because for any network $g$ where $(k, i)$ is not present, we have $\pi_{i}(g+(k, i))=\pi_{i}(g)+1$, hence also if an undirected path exists between $k$ and $i$. Property $\mathbf{D A}$ is clearly satisfied. Properties $\mathbf{B G}_{\mathbf{u}}$ and $\mathbf{R P}$ are satisfied because $\pi_{i}\left(g_{-i j}\right)=\pi_{i}\left(g_{-i k}\right)=1$ for any network $g$ and any two agents $j$ and $k$.

(all but $\mathbf{B G}_{\mathbf{u}}$ ) Let agent 1 be a special member of $N$, and let $i \in N \backslash\{1\}$. The following payoff function satisfies all properties, except $\mathbf{B G}_{\mathbf{u}}$ :

$$
\pi_{i}(g)= \begin{cases}-1 & \text { if } 1 \in N_{i}^{u}(g) \\ 0 & \text { otherwise }\end{cases}
$$

Property $\mathbf{B G}_{\mathbf{u}}$ is not satisfied because of the following. Let $g$ be a minimal network where $i$ observes set $S \not \supset 1$, where $i$ has no outgoing links, and where agent 1 is isolated. Then $\pi_{i}\left(g_{-i j}\right)=\pi_{i}\left(g_{-i}^{j}\right)=0$ for some $j \in S$. If an agent $r \in S$ adds $(1, r)$, then we obtain $\pi_{i}\left((g+(1, r))_{-i j}\right)=-1$ while $\pi_{i}\left((g+(1, r))_{-i}^{j}\right)=0$. Therefore, $\mathbf{B G}_{\mathbf{u}}$ is not satisfied. Property $\mathbf{D A}$ is clearly satisfied. Properties $\mathbf{N A}_{\mathbf{u}}$ and $\mathbf{R P}$ are satisfied, because neither an addition of a 'redundant' link nor a replacement of a link within the same component affects the set of observed agents.

(all but RP) Let agent 1 be a special member of $N$, and let $i \in N \backslash\{1\}$. The following payoff function satisfies all properties, except $\mathbf{R P}$ :

$$
\pi_{i}(g)= \begin{cases}1 & \text { if } 1 \in N_{i}^{d}(g) \text { and } 1 \notin N_{i}^{u}(g-(1, i)) \\ 0 & \text { otherwise }\end{cases}
$$

This payoff function does not satisfy $\mathbf{R P}$, because in any network $g_{-i}^{1}$, we have $\pi_{i}\left(g_{-i 1}\right)=1$, while $\pi_{i}\left(g_{-i j}\right)=0$ for each agent $j \in \operatorname{Car}\left(g_{-i}^{1}\right) \backslash\{1\}$. It can be verified that properties $\mathbf{N} \mathbf{A}_{\mathbf{u}}$ and $\mathbf{D A}$ are satisfied. Finally, property $\mathbf{B G}_{\mathbf{u}}$ is trivially satisfied because $\pi_{i}\left(g_{-i}^{j}\right)=0$ and $\pi_{i}\left(g_{-i j}\right) \geq 0$ for any network $g$ and any agent $j$. 


\subsection{Characterization}

Now we would like to know to what extent we generalized the existence result by Haller et al. (2007) for B\&G-2 functions. In other words, the question is which B\&G2 functions satisfy properties $\mathbf{D A}, \mathbf{N A}_{\mathbf{u}}, \mathbf{B G}_{\mathbf{u}}$, and $\mathbf{R P}$, and therefore imply the existence of local- and global-Nash networks.

It can easily be seen that $\mathbf{N} \mathbf{A}_{\mathbf{u}}$ is satisfied if and only if $c_{i j} \geq 0$, for all $i, j \in N$. We will refer to this condition as NNC (non-negative costs).

Further, property DA is clearly satisfied by all B\&G-2 functions.

The next lemma characterizes the B\&G-2 functions that satisfy property $\mathbf{R P}$.

Lemma 7.4.1. Let $\pi_{i}$ be a BEGG-2 function. Then $\pi_{i}$ satisfies $\boldsymbol{R P}$ if and only if the following property holds:

$\boldsymbol{P R P}$ If $c_{i j} \leq v_{i}(S)$ for agent set $S \subseteq N$ and agent $j \in S$, then for each $k \in S$ it holds that either $c_{i k}=c_{i j}$ or $c_{i k}>v_{i}(S)$.

Proof. Suppose that PRP is not satisfied. Then a set $S \subseteq N$ and agents $j, k \in S$ exist where $c_{i j} \leq v_{i}(S), c_{i k} \neq c_{i j}$, and $c_{i k} \leq v_{i}(S)$. Without loss of generality we may assume that $c_{i k}<c_{i j}$. Consider a minimal network $g$ where $i$ observes set $S$ in both $g_{-i j}$ and $g_{-i k}$. Hence, $\pi_{i}\left(g_{-i j}\right)=v_{i}(S)-c_{i j}<v_{i}(S)-c_{i k}=\pi_{i}\left(g_{-i k}\right)$, and therefore, $\mathbf{R P}$ is not satisfied.

Now suppose that RP is not satisfied. Then a minimal network $g$ exists, where $(j, i) \in g$, where $\pi_{i}\left(g_{-i j}\right) \geq \pi_{i}\left(g_{-i}^{j}\right)$, where $k \in \operatorname{Car}\left(g_{-i}^{j}\right)$, and where $\pi_{i}\left(g_{-i k}\right)>$ $\pi_{i}\left(g_{-i j}\right)$. Let $S$ be the set of agents that $i$ observes via $(j, i)$, i.e. $S=\operatorname{Car}\left(g_{-i}^{j}\right)$. Since $g$ is a minimal network, it follows that $\pi_{i}\left(g_{-i j}\right)-\pi_{i}\left(g_{-i}^{j}\right)=v_{i}(S)-c_{i j} \geq 0$, and hence that $c_{i j} \leq v_{i}(S)$. Since $0>\pi_{i}\left(g_{-i j}\right)-\pi_{i}\left(g_{-i k}\right)=c_{i k}-c_{i j}$, it follows that $c_{i k}<c_{i j}$. Therefore we have that $c_{i k} \leq v_{i}(S)$ and $c_{i k} \neq c_{i j}$; a contradiction with PRP.

The following lemma characterizes the set of B\&G-2 functions that satisfy $\mathbf{B G}_{\mathbf{u}}$.

Lemma 7.4.2. Let $\pi_{i}$ be a $B \mathscr{E} G$-2 function. Then $\pi_{i}$ satisfies $\boldsymbol{B G}_{\boldsymbol{u}}$ if and only if the following property holds:

$\boldsymbol{P B G}$ If $c_{i j} \leq v_{i}(S)$ for agent set $S \subset N$ and agent $j \in S$, then $c_{i j} \leq v_{i}\left(S^{\prime}\right)$ for all $S^{\prime} \supset S$.

Proof. First suppose that PBG does not hold. Then a set $S \subset N$, a set $S^{\prime} \supset S$, and an agent $j \in S$ exist such that $c_{i j} \leq v_{i}(S)$ and $c_{i j}>v_{i}\left(S^{\prime}\right)$. Consider a minimal network $g$ where $i$ has one incoming link, $(j, i)$, and no outgoing links. Thus, $\pi_{i}\left(g_{-i}^{j}\right)=0$. Furthermore, let all agent in $S$ form a component and all agents in $S^{\prime} \backslash S$ form a component. Let $k$ be an agent in $S$ and let $r$ be an agent in $S^{\prime}$. Observe that network 
$g+(k, r)$ is minimal. Since $(j, i) \in g$, and $j \in S$ we have $\pi_{i}\left(g_{-i j}\right)=v_{i}(S)-c_{i j} \geq 0$, and $\pi_{i}\left(g+(k, r)_{-i j}\right)=v_{i}\left(S^{\prime}\right)-c_{i j}<0$, while $\pi_{i}\left(g_{-i}^{j}\right)=\pi_{i}\left((g+(k, r))_{-i}^{j}\right)=0$. Hence, $\mathbf{B G}_{\mathbf{u}}$ is not satisfied.

Now suppose that $\mathbf{B G}_{\mathbf{u}}$ does not hold. Then a network $g$ exists such that link $(k, r) \notin g$, network $g+(k, r)$ is minimal, $\pi_{i}\left(g_{-i j}\right) \geq \pi_{i}\left(g_{-i}^{j}\right)$, and $\pi_{i}\left((g+(k, r))_{-i j}\right)<$ $\pi_{i}\left((g+(k, r))_{-i}^{j}\right)$. Let $S$ be the set of agents that $i$ observes in $g$ using link $(j, i)$. In network $g+(k, r)$, let $S^{\prime}$ be the set of agents that $i$ observes using $(j, i)$. Notice that since $g$ and $g+(k, r)$ are both minimal networks, $i$ uniquely observes $S$ and $S^{\prime}$ via $(j, i)$. We have $0 \leq \pi_{i}\left(g_{-i j}\right)-\pi_{i}\left(g_{-i}^{j}\right)=v_{i}(S)-c_{i j}$, and $0>\pi_{i}\left((g+(k, r))_{-i j}\right)-\pi_{i}\left((g+(k, r))_{-i}^{j}\right)=$ $v_{i}\left(S^{\prime}\right)-c_{i j}$. Hence we have obtained $c_{i j} \leq v_{i}(S)$ and $c_{i j}>v_{i}\left(S^{\prime}\right)$. Thus we conclude that $\mathbf{P B G}$ is not satisfied.

Hence, we obtain the following theorem from Lemma's 7.4.1 and 7.4.2, and from our observations that any B\&G-2 function satisfies DA and that $\mathbf{N A} \mathbf{A}_{\mathbf{u}}$ is satisfied if and only if link costs are non-negative (NNC).

Theorem 7.4.3. Let $\pi_{i}$ be a BEG-2 function. Then $\pi_{i}$ satisfies $\boldsymbol{D A}, \boldsymbol{N} \boldsymbol{A}_{\boldsymbol{u}}, \boldsymbol{B} \boldsymbol{G}_{\boldsymbol{u}}$, and $\boldsymbol{R P}$ if and only if

$$
(\boldsymbol{N N C}) \quad c_{i j} \geq 0 \quad \forall j \in N \text {, and }
$$

if $c_{i j} \leq v_{i}(S)$ for agent set $S \subseteq N$ and agent $j \in S$ then

$$
\begin{array}{lll}
(\boldsymbol{P B G}) & c_{i j} \leq v_{i}\left(S^{\prime}\right) & \forall S^{\prime} \supseteq S, \\
(\boldsymbol{P R P}) & c_{i k}=c_{i j} \text { or } c_{i k}>v_{i}(S) & \forall k \in S .
\end{array}
$$

By Theorem 7.2.3 and Corollary 7.2.4, we know that local- and global-Nash networks respectively exist for all joint payoff functions that satisfy $\mathbf{D A}, \mathbf{N A} \mathbf{A}_{\mathbf{u}}, \mathbf{R P}$, and $\mathbf{B G}_{\mathbf{u}}$. Hence we obtain the following result.

Corollary 7.4.4. For joint BEG-2 functions that satisfy $\boldsymbol{N} \boldsymbol{N} \boldsymbol{C}, \boldsymbol{P B} \boldsymbol{G}$, and $\boldsymbol{P} \boldsymbol{R} \boldsymbol{P}$, local-Nash and global-Nash networks exist.

Therefore, we know that local- and global-Nash networks also exist for (some) joint B\&G-2 functions with negative profits. Recall from Chapter 6 that we did not manage to prove the existence of global-Nash networks for B\&G-1 functions with negative profits (see Corollary 6.3.3.

In Figure 7.5 we show the characterization of B\&G-2 functions graphically. Each area corresponds to a specific subset of B\&G-2 functions. Area 8 corresponds to B\&G2 functions that satisfy NNC, PBG, and PRP. Recall that each B\&G-2 function trivially satisfies DA. Therefore, local- and global-Nash networks exist for each game where each agent $i$ has a B\&G-2 function in area 8 . 


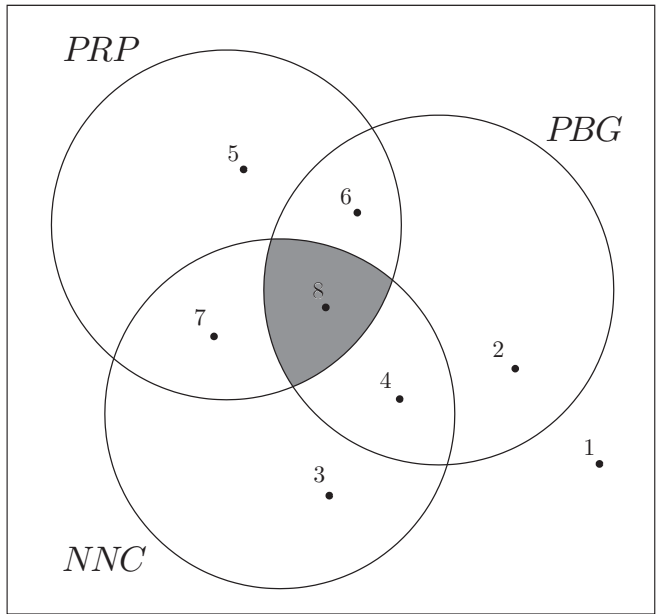

Figure 7.5: The characterization of B\&G-2 functions.

For each area, an example of a B\&G-2 function is given in Table 7.1. In these examples, three agents are involved, except for area 3 where four agents are involved 1

\begin{tabular}{llllrrrrrr}
\hline \multirow{2}{*}{ Area } & \multicolumn{1}{l}{ Properties } & \multicolumn{9}{c}{ Example } & & & \\
& PRP & NNC & PBG & $c_{i j}$ & $c_{i k}$ & $c_{i \ell}$ & $v_{i j}$ & $v_{i k}$ & $v_{i \ell}$ \\
\hline 1 & $\mathrm{n}$ & $\mathrm{n}$ & $\mathrm{n}$ & -1 & 0 & & 0 & -2 & \\
2 & $\mathrm{n}$ & $\mathrm{n}$ & $\mathrm{y}$ & -1 & 3 & & 2 & 1 & \\
3 & $\mathrm{n}$ & $\mathrm{y}$ & $\mathrm{n}$ & 0 & 1 & 0 & 1 & 1 & -1 \\
4 & $\mathrm{n}$ & $\mathrm{y}$ & $\mathrm{y}$ & 0 & 1 & & 0 & 1 & \\
5 & $\mathrm{y}$ & $\mathrm{n}$ & $\mathrm{n}$ & -1 & -1 & & 0 & -2 & \\
6 & $\mathrm{y}$ & $\mathrm{n}$ & $\mathrm{y}$ & -2 & -2 & & 0 & 1 & \\
7 & $\mathrm{y}$ & $\mathrm{y}$ & $\mathrm{n}$ & 2 & 2 & & 2 & -1 & \\
8 & $\mathrm{y}$ & $\mathrm{y}$ & $\mathrm{y}$ & 2 & 2 & & 3 & -1 & \\
\hline
\end{tabular}

Table 7.1: The eight area's in Figure 7.5

In the following result, we show that PBG and PRP imply that links can be divided in two groups: one with affordable, owner-homogeneous links, and one with unaffordable links (i.e. $c_{i j}>v_{i}(N)$ ).

Proposition 7.4.5. Let $\pi_{i}$ be a BEG-2 function that satisfies $\boldsymbol{P B G}$ and $\boldsymbol{P R P}$. If $c_{i j} \leq v_{i}(S)$ for a set $S$ and an agent $j \in S$, then for each $k \in N$ either $c_{i k}=c_{i j}$ or $c_{i k}>v_{i}(N)$.

\footnotetext{
${ }^{1}$ It can be verified that an example with three agents for area 3 does not exist.
} 
Proof. By PBG it follows directly that if $c_{i j} \leq v_{i}(S)$ for some agent $j$ and set $S$, then $c_{i j} \leq v_{i}(N)$. By PRP this implies that for each $k \in N$, either $c_{i k}=c_{i j}$ or $c_{i k}>v_{i}(N)$.

Thus, our approach generalizes Haller et al. (2007), because we proved the existence of global-Nash networks for games where profits may be negative, and where link costs are either affordable and owner-homogeneous, or unaffordable.

However, our framework does not cover the class of B\&G-2 functions for which Kamphorst and Van der Laan (2007) prove the existence of Nash networks. Profits are homogeneous in those functions, and link costs are heterogeneous under the following conditions. Agents are divided into groups that are located on a line. If agents $i$ and $j$ belong to the same group, then the cost of link $(j, i)$ is a fixed positive value, and if they belong to different groups, this cost is strictly increasing in the distance between these two groups. It is easily verified that those B\&G-2 functions satisfy the properties DA, $\mathbf{N A}_{\mathbf{u}}$, and $\mathbf{B G}_{\mathbf{u}}$, but do not satisfy $\mathbf{R P}$. For future research, it would be worthwhile to develop axiomatic properties that cover those B\&G-2 functions.

Besides B\&G-2 functions, also other payoff functions exist that satisfy our framework of payoff properties, for instance:

$$
\begin{aligned}
& \pi_{i}(g)=\left|K_{i}^{u}(g)\right|-\left|N_{i}^{d}(g)\right|, \\
& \pi_{i}(g)=\left|C^{u}\left(g_{-i}\right) \cap N_{i}^{u}(g)\right|-\left|N_{i}^{d}(g)\right|,
\end{aligned}
$$

where $K_{i}^{u}(g)$ is the number of spokes that $i$ observes in $g$ (link $(k, r)$ is called a spoke in $g$ if there exists a cycle in $g-(k, r)$ containing both $k$ and $r)$ and $C^{u}(g)$ is the number of agents that are contained in an undirected cycle in $g$. These two payoff functions satisfy all properties, i.e. $\mathbf{D A}, \mathbf{N A}_{\mathbf{u}}, \mathbf{B G}_{\mathbf{u}}$, and $\mathbf{R P}$. They fall outside the class of B\&G-2 functions, because B\&G-2 functions only take the sets $N_{i}^{u}(g)$ and $N_{i}^{d}(g)$ into account, while the payoff functions $(7.10$ and 7.11 also take other properties of the network architecture into account.

\subsection{Concluding remarks}

In this chapter, we proposed a framework of payoff properties that covers the twoway flow model, in the same way as we did in Chapter 4 for the one-way flow model. We proved the existence of local- and global-Nash networks for B\&G-2 functions with owner-homogeneous link costs, and where profits may be negative. Further, also nonB\&G-2 functions exist that satisfy our properties, for instance the ones defined in 7.10 and 7.11).

In Chapter 9, we discuss the property frameworks proposed in this chapter and in Chapter 4 and relate them to models with information decay and imperfectly reliable links. 


\section{Chapter 8}

\section{Project Support Games}

In economic situations, one often encounters a large project that has to be financed by several parties. Each party benefits from completing the project. The question is whether the parties are willing to invest in the project, and if so, how much. In this chapter, we study so-called project support games in order to answer these questions.

Although project support games do not seem to be related to network formation at first glance, they are inspired by a model of network formation proposed by Bloch and Jackson (2007). In that model, agents make investments (positive or negative) in each link. Whenever the investments in a link are non-negative, this link will be formed. Instead of the formation of a network consisting of links, we study the financing of a project consisting of tasks.

Let a project support game be defined by a set of agents, a set of tasks, a cost function, and a utility profile. A project consists of several tasks, each of them having its own development cost. However, since these costs are related to each other, we define a cost function on the set of all projects. When a project is financed and executed, each agent receives a utility.

We discuss the following approach. Simultaneously, the agents propose an investment in each task. These investments may be negative, which means that agents can make demands instead of offers. A project is called feasible if all investments in it together cover its cost. For the feasible project that is executed, each agent receives his utility, and pays his proposed investments in the tasks of that project.

Stability is the property of an investment profile so that in case the grand project (i.e. the project containing all tasks) is executed, there is no incentive to deviate. We provide necessary and sufficient conditions for which stable investments exist.

We relate stability to the concept of Nash equilibria. The investments of an agent can be seen as his action, and the investment profile as the actions of all agents together. A feasible project for which the aggregate utility minus its cost is maximal, is actually executed. For this project, each agent receives his payoff, that is his utility minus his proposed investments in the tasks of that project. We show that a stable investment 
profile is a Nash equilibrium where the grand project is executed.

Our approach is purely theoretical. Step-level public goods games are studied in the literature to examine this subject from an experimental point of view. An overview of those games is provided by Schram et al. (2008).

This chapter is outlined as follows. In Section 8.1 we introduce the model. Then, in Section 8.2 we provide conditions for which stable investments exist. By means of examples, we show in Section 8.3 how project support games can be applied in specific economic situations. In Section 8.4 we relate stability to the Nash equilibrium concept. Finally, in Section 8.5 we discuss the model and relate it to the literature.

This chapter is based on Derks et al. (2009b).

\subsection{Model}

Let $(N, E, c, u)$ denote a project support game, where $N$ is a fixed set of agents and $E=\left\{e_{1}, \ldots, e_{m}\right\}$ is a set of tasks. A subset of $E$ is called a project and the set of projects is denoted by $2^{E}$. Further, $c: 2^{E} \rightarrow \mathbb{R}$ is a cost function, and $u=\left(u_{i}\right)_{i \in N}$ is a utility profile where $u_{i}: 2^{E} \rightarrow \mathbb{R}$ is the utility function of each agent $i \in N$. Observe that the utilities and the cost of the empty project may be non-zero.

The question that we address in this chapter is whether the agents are willing to invest in the grand project $E$. If for instance, $c(E)=c(P)$ while $u_{i}(E)<u_{i}(P)$, then agent $i$ prefers project $P$ over $E$, and therefore he will be reluctant to invest in the tasks in $E \backslash P$.

The investments of the agents are modeled as follows. Let $t=\left(t_{e}^{i}\right)_{i \in N, e \in E}$ be an investment profile, where $t_{e}^{i}$ is the investment of agent $i$ in task $e$. Project $P \subseteq E$ is called $t$-feasible if

$$
\sum_{i \in N} \sum_{e \in P} t_{e}^{i} \geq c(P)
$$

(here we assume that summation over an empty set is zero, so that the empty project is feasible for any investment profile when $c(\emptyset) \leq 0)$.

Given an investment profile $t$, a $t$-feasible project $P \subseteq E$ will be executed, and the gain for each agent $i$ is then given by:

$$
u_{i}(P)-\sum_{e \in P} t_{e}^{i}
$$

We do not yet explicitly model which feasible project will be executed (in Section 8.4 we do). The reason for this is that we are interested whether investments can be found such that there is no incentive to deviate from the grand project $E$. This property is called stability and is covered in the following section. 


\subsection{Stability}

In this section we are looking for an investment profile $t$ such that any deviation from the grand project $E$ is not profitable.

Consider an agent $i$. Given the investments of all other agents, the minimum value of investments that $i$ has to propose in order to make project $E$ feasible is

$$
c(E)-\sum_{j \neq i} \sum_{e \in E} t_{e}^{j}
$$

This can be seen as: agent $i$ pays the total cost for the grand project $E$, but he is sponsored by the other agents. In this case, $i$ 's gain is given by

$$
u_{i}(E)-c(E)+\sum_{j \neq i} \sum_{e \in E} t_{e}^{j}
$$

This analysis can be applied to any project $P \subseteq E$. This brings us to the following observation. Grand project $E$ is preferred over any other project $P \subset E$ whenever

$$
u_{i}(E)-c(E)+\sum_{j \neq i} \sum_{e \in E} t_{e}^{j} \geq u_{i}(P)-c(P)+\sum_{j \neq i} \sum_{e \in P} t_{e}^{j} \quad \forall i, \forall P \subset E .
$$

The left-hand side and the right-hand side correspond to the gain of agent $i$ when respectively $E$ and $P \subset E$ are executed.

We want to find investments that satisfy 8.2 such that the sum of these investments equals the cost of $E$. Consider the following constraint:

$$
\sum_{i \in N} \sum_{e \in E} t_{e}^{i} \leq c(E)
$$

This constraint may look contra-intuitive at first glance, because we rather expect the total investment to be greater or equal than the total cost. But if the total investment is strictly less than $c(E)$, then the investments can be raised as follows:

$$
t_{e}^{\prime i}=t_{e}^{i}+\frac{c(E)-\sum_{j \in N, e^{\prime} \in E} t_{e^{\prime}}}{n|E|} \quad \text { for all } i \in N, e \in E .
$$

If $t$ satisfies 8.2 , then $t^{\prime}$ also satisfies 8.2 , because the left-hand side of this inequality is raised at least as much as its right-hand side. By (8.4), it follows directly that if $t$ satisfies 8.3 , then $t^{\prime}$ satisfies 8.3 with equality.

An investment profile $t$ is called stable if 8.2 and 8.3 hold. To find stable investments, we can solve the following LP problem:

$$
\begin{aligned}
& \text { minimize } \sum_{i \in N} \sum_{e \in E} t_{e}^{i} \\
& \text { subject to } 8.2 .
\end{aligned}
$$


Stable investments exist if and only if the optimum value is at most $c(E)$. More specifically, the optimal solutions of the LP problem are stable if and only if (8.3) holds. If the optimum value is less than $c(E)$, then we can apply (8.4) to obtain stable investments that are equal to $c(E)$.

Observe from 8.2 that each agent $i$ is only interested in the total investments of the other agents w.r.t. a single task, and not in the individual investments. Therefore, we define a profile $q=\left(q_{e}^{i}\right)_{i \in N, e \in E}$ with

$$
q_{e}^{i}=\sum_{j \neq i} t_{e}^{j} \quad \forall i \in N, \forall e \in E
$$

Thus, $q_{e}^{i}$ is equal to the total investment of all agents in $N \backslash\{i\}$ on task $e$.

Now, we show that there is a one-to-one correspondence between the $t$ and $q$ profiles with this substitution. By 8.5 we know that each $t_{e}^{i}$ is contained in $(n-1)$ values of $q$, namely in each $q_{e}^{j}$ where $j \neq i$. Therefore we have

$$
\sum_{i \in N} t_{e}^{i}=\frac{\sum_{j \in N} q_{e}^{j}}{n-1}
$$$$
\forall e \in E \text {. }
$$

Writing the left-hand side as $t_{e}^{i}+\sum_{j \neq i} t_{e}^{j}=t_{e}^{i}+q_{e}^{i}$ for each agent $i$, we obtain

$$
t_{e}^{i}=\frac{\sum_{j \in N} q_{e}^{j}}{n-1}-q_{e}^{i} \quad \forall i \in N, \forall e \in E .
$$

Hence, we conclude that there is a one-to-one correspondence between $t$ and $q$.

By the substitution of $t$ with $q$, constraints 8.2 can be rewritten as

$$
u_{i}(E)-c(E)+\sum_{e \in E} q_{e}^{i} \geq u_{i}(P)-c(P)+\sum_{e \in P} q_{e}^{i} \quad \forall i \in N, \forall P \subset E .
$$

Observe that, in contrast with 8.2 , only variables regarding agent $i$ are contained in (8.8.

We obtain 8.3 in terms of $q$ by the following derivation

$$
\begin{gathered}
\sum_{i \in N} \sum_{e \in E} q_{e}^{i}=\sum_{i \in N} \sum_{e \in E} \sum_{j \neq i} t_{e}^{j}=\sum_{e \in E} \sum_{j \in N}(n-1) t_{e}^{j} \\
=\left(\sum_{i \in N} \sum_{e \in E} t_{e}^{i}\right)(n-1) \leq(n-1) c(E) .
\end{gathered}
$$

Hence, in order to find stable investments, we have to find a $q=\left(q_{e}^{i}\right)_{i \in N, e \in E}$ such that conditions 8.8 and 8.9 hold. This can be done by minimizing $\sum_{e \in E} q_{e}^{i}$ for each agent $i$ separately: 


$$
\begin{array}{cl}
\operatorname{minimize} & \sum_{e \in E} q_{e}^{i} \\
\text { subject to } & u_{i}(E)-c(E)+\sum_{e \in E} q_{e}^{i} \geq u_{i}(P)-c(P)+\sum_{e \in P} q_{e}^{i} \quad \forall P \subset E .
\end{array}
$$

Thus we obtained a decomposition into agent separated LP problems.

Consider $u_{i}-c$ as a set function. Observe that its value for the empty project may not be equal to 0 , which is for example the case when setup costs are involved. In case the value of $u_{i}-c$ with respect to the empty project equals 0 , then this set function is equivalent to the characteristic function of a cooperative game. When adding an appropriate additive gam $\AA^{1}$ to $u_{i}-c$, the obtained set function (or game) will be grand coalition monotone $(\mathrm{GCM})$, i.e. the value for the grand coalition (in our case, $E$ ) is greater than or equal to the value for any other coalition.

The constraints of the LP problem 8.10 express the GCM property, where $q$ can be seen as an additive game. Let $M\left(u_{i}-c\right)$, the GCM value of set function $u_{i}-c$, be equal to the optimum value of LP problem 8.10. By 8.9 we obtain the following theorem:

Theorem 8.2.1. A stable investment profile $t$ exists if and only if

$$
\sum_{i \in N} M\left(u_{i}-c\right) \leq(n-1) c(E) .
$$

The intuition of Theorem 8.2.1 is as follows. If agent $i$ is responsible for the costs, then his actual gain is $u_{i}-c$. The other agents may contribute to the costs in such a way that the grand project $E$ is the most beneficial outcome for $i$. In case the agents' influence on the costs is configured by contributions on the level of the tasks, then $M\left(u_{i}-c\right)$ is the minimal total contribution such that the grand project is the most profitable project for $i$. So, for realizing the grand project, agent $i$ needs to contribute at least $c(E)-M\left(u_{i}-c\right)$, and 8.11 now states that the total of these contributions, over all agents, should be at least the cost of the grand project:

$$
\sum_{i \in N}\left(c(E)-M\left(u_{i}-c\right)\right) \geq c(E) .
$$

When the latter inequality does not hold, and the realization of the grand project is desirable, then the present situation has to be extended with new agents $k$ with a utility function $u_{k}$ that fulfills $M\left(u_{k}-c\right)<c(E)$; that is, if the other agents only need to invest an amount less than the cost of the grand project in order to let the new agent $k$ be interested in the grand project, then letting $k$ participate makes the left-hand side of 8.12 grow and thus benefits the realization of the grand project.

\footnotetext{
${ }^{1} \mathrm{~A}$ cooperative game is called additive if it has a characteristic function $v: 2^{E} \rightarrow \mathbb{R}$ that satisfies $v(S \cup T)=v(S)+v(T)$ for each $S, T \subseteq E$ with $S \cap T=\emptyset$.
} 
Conditions for utility and cost functions $u_{i}$ and $c$ are easily established for having the inequality $M\left(u_{i}-c\right)<c(E)$, especially when $c$ possesses a non-empty anti-core (that is, there exists a cost distribution $x \in \mathbb{R}^{E}$ with $\sum_{e \in E} x_{e}=c(E)$ and $\sum_{e \in P} x_{e} \leq c(P)$ for all $P \subseteq E$ ). A supermodular utility function and a submodular cost function may not be sufficient, as the following example shows ${ }^{2}$

Example 8.2.2. Let all utilities be equal to zero, i.e. let $u_{i}(P)=0$ for all $i \in N$ and all $P \subseteq E$. Further, let the cost of project $P \subseteq E$ be equal to the number of tasks in $P$, i.e. let $c(P)=|P|$. Observe that $x_{e}=1$ for all $e \in E$ is an anti-core element of $c$.

Stable investments do not exist for this example, because of the following. The constraint of LP problem 8.10 with $P=\emptyset$ yields $\sum_{e \in E} q_{e}^{i} \geq|E|$ for each agent $i \in N$. Hence

$$
\sum_{i \in N} M\left(u_{i}-c\right) \geq \sum_{i \in N} \sum_{e \in E} q_{e}^{i} \geq n c(E) .
$$

Since this is in contradiction with 8.11), we conclude that stable investments do not exist for this example.

\subsection{Examples}

In this section we provide two examples that illustrate how project support games can be applied. The first one illustrates how stable investments can be found concretely, and the second one describes a real-life application of project support games.

Example 8.3.1. Let $N=\{1,2\}$ be the set of agents and let $E=\{A, B\}$ be the set of tasks. Consider the following costs and utilities.

\begin{tabular}{crrr}
\hline$P$ & $c(P)$ & $u_{1}(P)$ & $u_{2}(P)$ \\
\hline$\emptyset$ & 1 & 0 & 0 \\
$\{A\}$ & 15 & 7 & 10 \\
$\{B\}$ & 15 & 8 & 4 \\
$\{A, B\}$ & 20 & 10 & 11 \\
\hline
\end{tabular}

Table 8.1: The costs and utilities of Example 8.3.1

First, we solve LP 8.10 with respect to agent 1:

$$
\begin{aligned}
& \text { minimize } q_{A}^{1}+q_{B}^{1} \\
& \text { subject to } 10-20+q_{A}^{1}+q_{B}^{1} \geq \begin{cases}0-1 & \text { for } P=\emptyset \\
7-15+q_{A}^{1} & \text { for } P=\{A\} \\
8-15+q_{B}^{1} & \text { for } P=\{B\}\end{cases}
\end{aligned}
$$

\footnotetext{
${ }^{2}$ Supermodular and submodular set functions are defined at the bottom of page 38
} 
It can easily be verified that the solutions are described by $q_{A}^{1}+q_{B}^{1}=9$ where $q_{A}^{1} \geq 3$ and $q_{B}^{1} \geq 2$. Take the solution $q_{A}^{1}=5$ and $q_{B}^{1}=4$.

The LP with respect to agent 2 is the following:

$$
\begin{aligned}
& \text { minimize } q_{A}^{2}+q_{B}^{2} \\
& \text { subject to } 11-20+q_{A}^{2}+q_{B}^{2} \geq \begin{cases}0-1 & \text { for } P=\emptyset \\
10-15+q_{A}^{2} & \text { for } P=\{A\} \\
4-15+q_{B}^{2} & \text { for } P=\{B\}\end{cases}
\end{aligned}
$$

Here, the solutions are given by $q_{A}^{2}+q_{B}^{2}=8$ where $q_{A}^{2} \geq-2$ and $q_{B}^{2} \geq 4$. Take the solution $q_{A}^{2}=3$ and $q_{B}^{2}=5$.

Since $n=2$ it follows directly from 8.5 that $t_{A}^{1}=q_{A}^{2}$, etcetera. Since the sum over all $t$ 's is equal to 17 , and the cost for $\{A, B\}$ is 20 , each $t$ is raised by 0.75 according to (8.4). Hence the following stable investments are obtained:

$$
t=\left(\begin{array}{cc}
t_{A}^{1} & t_{A}^{2} \\
t_{B}^{1} & t_{B}^{2}
\end{array}\right)=\left(\begin{array}{ll}
3.75 & 5.75 \\
5.75 & 4.75
\end{array}\right)
$$

Observe that stable investments exist although the aggregate utility minus the cost of project $\{A\}$ is strictly higher than that of the grand project. However, project $\{A\}$ is not $t$-feasible, and neither agent 1 nor agent 2 can improve by deviating in order to make $\{A\}$ feasible.

Example 8.3.2. Among the people in a local area, there is a growing interest in having an own neighborhood center. In this center, associations have the opportunity to employ various activities. The local authority decides to build this center only if there is enough support among the associations. The building and maintenance costs are discounted into monthly payments. Associations can rent the center from the local authority.

Based on how much rent the associations are willing to pay for the center, the local authority will decide whether to build the center, and if this is the case, which facilities will be included. Consider the following facilities: a tavern, a meeting room, a music hall, and sports facilities. The following parties are interested in renting the center: an athletic club, a church community, a drama club, and a blues band.

Each party has a priority list of the facilities. The athletic club gives priority to the sports facilities and the tavern, the church community will use the music hall and the meeting room most frequently, the drama club all of them, and the blues band the music hall and occasionally the tavern. These priorities are translated into utility functions. It is intuitive to assume that these utilities are monotone, e.g. the athletic club will also benefit from a meeting room.

The building and maintenance costs depend on which facilities the center will have. In a situation like this, the cost function is normally submodular: building a center with only a meeting room is relatively very expensive. Furthermore, if the local authority 
would like to take setup costs into account, then this is reflected in a cost function $c$ with $c(\emptyset)>0$.

Based on the utility and cost functions, the investments can be determined. An investment $t_{e}^{i}$ corresponds to the investment proposed by party $i$ in facility $e$. If the investments are stable, then the local authority will build the whole center, and rent it to the parties. The amount of rent paid by party $i$ corresponds to the sum of investments proposed by party $i$.

Observe that it could be the case that the total investment on a certain facility, for instance the meeting room, may be negative even though the whole center is built. In that case, the surplus of investments on the other facilities is used to finance the additional cost of building the meeting room.

\subsection{Nash equilibria}

In this section we show that stability is directly related to the notion of Nash equilibria with respect to the grand project $E$.

For each agent $i$, consider $t^{i}=\left(t_{e}^{i}\right)_{e \in E}$ as an action of agent $i$. In order to define a payoff function given an investment profile $t$, we have to model which $t$-feasible project is executed. We assume here that $c(\emptyset)=0$, for otherwise $t$-feasible projects may not exist. A $t$-feasible project $P$ for which $\left(\sum_{i \in N} u_{i}(P)-c(P)\right)$ is maximal will clearly be of interest among the agents. Observe that there may be more than one candidate that fulfills this property. Let the set of those candidates be defined as

$$
\mathcal{P}(t)=\underset{t \text {-feasible } P}{\operatorname{argmax}}\left(\sum_{i \in N} u_{i}(P)-c(P)\right) .
$$

Let $\phi: 2^{E} \rightarrow \mathbb{N}$ be a ranking function. Given an investment profile $t$, let the project that is executed be defined as

$$
P(t)=\underset{P \in \mathcal{P}(t)}{\operatorname{argmin}}(\phi(P))
$$

Each agent $i$ receives the following payoff:

$$
\pi_{i}(t)=u_{i}(P(t))-\sum_{e \in P(t)} t_{e}^{i}
$$

The investment profile $t$ is called a Nash equilibrium if

$$
\pi_{i}(t) \geq \pi_{i}\left(\hat{t}^{i}, t_{-i}\right)
$$

for each agent $i$ and investments $\hat{t}^{i}$, where $t_{-i}=\left(t^{j}\right)_{j \in N \backslash\{i\}}$. By 8.14 we know that investment profile $t$ is a Nash equilibrium if

$$
u_{i}(P(t))-\sum_{e \in P(t)} t_{e}^{i} \geq u_{i}\left(P\left(\hat{t}^{i}, t_{-i}\right)\right)-\sum_{e \in P\left(\hat{t}^{i}, t_{-i}\right)} \hat{t}_{e}^{i} \quad \forall i, \forall \hat{t}^{i} .
$$


It can easily be verified that a Nash equilibrium $t$ has the following property:

$$
\sum_{i \in N} \sum_{e \in P(t)} t_{e}^{i}=c(P(t))
$$

implying that the total investment of each agent $i$ is equal to

$$
c(P(t))-\sum_{j \neq i} \sum_{e \in P(t)} t_{e}^{j}
$$

Therefore, 8.15 can be rewritten as

$$
u_{i}(P(t))-c(P(t))+\sum_{j \neq i} \sum_{e \in P(t)} t_{e}^{j} \geq u_{i}\left(P\left(\hat{t}^{i}, t_{-i}\right)\right)-\sum_{e \in P\left(\hat{t}^{i}, t_{-i}\right)} \hat{t}_{e}^{i} \quad \forall i, \forall \hat{t}^{i} .
$$

For this condition, only deviations $\hat{t}^{i}$ have to be considered that make $P\left(\hat{t}^{i}, t_{-i}\right)$ exactly feasible, i.e.

$$
\sum_{e \in P\left(\hat{t}^{i}, t_{-i}\right)} \hat{t}_{e}^{i}=c\left(P\left(\hat{t}^{i}, t_{-i}\right)\right)-\sum_{j \neq i} \sum_{e \in P\left(\hat{t}^{i}, t_{-i}\right)} t_{e}^{j}
$$

Hence, $t$ is a Nash equilibrium if

$u_{i}(P(t))-c(P(t))+\sum_{j \neq i} \sum_{e \in P(t)} t_{e}^{j} \geq u_{i}\left(P\left(\hat{t}^{i}, t_{-i}\right)\right)-c\left(P\left(\hat{t}^{i}, t_{-i}\right)\right)+\sum_{j \neq i} \sum_{e \in P\left(\hat{t}^{i}, t_{-i}\right)} t_{e}^{j} \forall i, \forall \hat{t}^{i}$.

Observe that $\hat{t}^{i}$ only plays a role in determining the project $P\left(\hat{t}^{i}, t_{-i}\right)$, i.e. the project that is executed when the agents play $\left(\hat{t}^{i}, t_{-i}\right)$. Therefore, for $P(t)=E$, condition 8.18 is implied by 8.2 , where all projects $P \subseteq E$ are examined. We conclude that $t$ constitutes a Nash equilibrium in the case $t$ is stable.

However, the other way round does not necessarily hold, because inequality (8.2) applies to all projects $P \subseteq E$, instead of only the projects that are executed in case of deviation. Consider for instance an agent $i$ and a deviation $\hat{t}^{i}$. It may be the case that 8.18 holds while

$$
u_{i}(P(t))-c(P(t))+\sum_{j \neq i} \sum_{e \in P(t)} t_{e}^{j}<u_{i}(P)-c(P)+\sum_{j \neq i} \sum_{e \in P} t_{e}^{j},
$$

for some $\left(\hat{t}^{i}, t_{-i}\right)$-feasible project $P$ other than $P\left(\hat{t}^{i}, t_{-i}\right)$. In that case, 8.2 does not hold.

\subsection{Discussion}

The presented model is inspired by a model of network formation proposed by Bloch and Jackson (2007). In that model, the agents make investments in the links. Negative investments (i.e. demands) are also allowed. A link will be formed if the total 
investment is non-negative. They consider two settings. One where agents can only invest in adjacent links, and one where agents can also make offers (i.e. non-negative investments) to nonadjacent links.

In our model, agents do not form a network consisting of links, but a project consisting of tasks. Each agent proposes an investment in each task, whereas in the model by Bloch and Jackson (2007), each agent proposes an investment in each adjacent link. This aspect can be implemented in our model in the following way. Let $E^{i} \subseteq E$ be the personal project of agent $i \in N$, and let $E^{-i}=E \backslash E^{i}$ be the part of the grand project in which $i$ is not involved. Agent $i$ is only allowed to invest in tasks of his personal project. Each task in the grand project belongs to at least one of the personal projects, i.e. $\bigcup_{i \in N} E^{i}=E$.

Unfortunately, our analysis cannot easily be applied to the model with personal projects. The main reason for this, is that the substitution of $t$ with $q$ does not yield a decomposition of agent independent minimization problems (see (8.10)). Therefore, a different approach is required to find stable investments in that case.

Another difference with the approach by Bloch and Jackson (2007) is the way how costs are modeled. The link costs in the model by Bloch and Jackson (2007) are included in the utilities. However, a link is only formed if the total investments in it are non-negative. This is equivalent as defining the link costs to be 0 . We explicitly incorporate costs for executing projects. Observe that these costs are defined on the set of projects. So, the proposed investments in an individual task do not have to cover a specific cost. When we add such constraints to our model, a different analysis is required to find stable investments.

Koster et al. (2003) propose a model that is also related to ours. They consider a cost function that is defined on the set of tasks. Each agent has a personal project, which has to be executed in order to receive a utility. Another model that is related to ours is proposed by Tijs and Brânzei (2004). In their model, the cost function is also defined on the set of tasks. Their utilities functions are similar to ours. Both Koster et al. (2003) and Tijs and Brânzei (2004) study a cooperative game, that is extracted from the model, for which they prove convexity.

In case we want to know whether investments can be found that are stable with respect to a specific project $T \subset E$, our analysis can be applied to $T$ instead of $E$. One problem that occurs here is which $t$-feasible project will be executed. Similar to Koster et al. (2003) and Tijs and Brânzei (2004), we define $P(t)$ to be the project such that $\left(\sum_{i \in N} u_{i}(P)-c(P)\right)$ is maximal over all feasible projects $P$. Although this is very reasonable, it could be the case that some agents still prefer other $t$-feasible projects.

In conclusion, unlike the other models, our model takes cost functions into account that do not have to be linear. Further, not every specific task has to be financed directly, that is, we do not restrict to $\sum_{i \in N} t_{e}^{i} \geq c(\{e\})$ for each task $e$. For further research, it is interesting to study our model more extensively, for instance, by enhancing it with personal projects. 


\section{Chapter 9}

\section{Conclusion}

In this chapter, we provide concluding remarks and recommendations for further research. We discuss the results with respect to the unilateral connections model in Section 9.1. Finally, we provide directions for further research in Section 9.2 .

\subsection{Unilateral connections model}

In Chapters 2 to 7, we studied models of unilateral network formation. The basic model that we built on, is proposed by Bala and Goyal (2000a). Each agent chooses a set of own links, and all these links together define the outcome network which yields a payoff for each agent. This payoff function consists of a cost and a profit part. Each agent pays a certain cost for each formed link. As for the profits, several variants are studied in the literature, which are characterized by the following settings:

Information flow (one-way or two-way) Let an observation path be a path via which one agent observes another. In the one-way flow model, this path is directed from the observed agent to the observer, and in the two-way flow model this path is undirected.

Information decay The value of profits that agent $i$ receives from observing $j$ depends on the distance $d_{i j}$, which is the number of links on the shortest observation path. More specifically, agent $i$ receives $\delta^{d_{i j}} v_{i j}$, where $\delta$ is a decay factor (normally between 0 and 1 ).

Link reliability Each link $(j, i)$ is reliable with probability $p_{i j}$. The value of profits that $i$ receives from observing $j$ is multiplied by the probability that all links of at least one observation path are reliable.

We identify a specific variant by either B\&G-1 or B\&G-2, optionally with the addition of a ' $d$ ' for decay and/or an ' $r$ ' for imperfect link reliability. For example, by 
B\&G-2r we mean the two-way flow model with imperfectly reliable links and without decay. Observe that we may identify these models by the corresponding classes of payoff functions.

In Chapter 2, we extensively studied the B\&G-1 model (that is, the one-way flow model without decay and with perfectly reliable links). We examined the existence and architecture of Nash network. The results are summarized in Table 2.1 on page 34.

Further, we introduced axiomatic payoff properties for these models. In Chapters 4 and 7 we proposed two frameworks of properties that cover the B\&G-1 and the B\&G-2 model respectively. These properties (except disjoint additivity) are oriented on local actions, i.e. they compare two networks that are interchangeable by a local action.

We developed the frameworks in such a way that they are as general as possible, and that they guarantee the existence of Nash networks. Observe that our main focus in this thesis is on the existence rather than on the architecture of Nash networks. Interestingly, the architecture of Nash networks often follows directly from the proofs of existence (see Corollary 2.2.3 and Theorems 2.2.8, 4.3.1, and 7.2.3). Proper networks (e.g. Figure 2.10 are essential in the one-way flow model and minimal networks (e.g. Figure 7.2 in the two-way flow model.

In Table 9.1, we show which payoff properties that we introduced are satisfied by which variants. Here, we divide the properties into four categories based on their function. We leave out properties $\mathbf{B T}$ and $\mathbf{B F}$ since they are derived from and implied by DE. In this table, link costs are owner-homogeneous and profits are heterogeneous.

\begin{tabular}{|c|c|c|c|c|c|c|}
\hline & B\&G-1 & B\&G-1d & B\&G-1r & B\&G-2 & $\mathrm{B} \& \mathrm{G}-2 \mathrm{~d}$ & B\&G-2r \\
\hline \multicolumn{7}{|c|}{ Treatment of different $i$-disjoint networks } \\
\hline DA & $\checkmark$ & $\checkmark$ & $\checkmark$ & $\checkmark$ & $\checkmark$ & $\checkmark$ \\
\hline \multicolumn{7}{|c|}{ Addition/deletion of a link } \\
\hline NA & $\checkmark$ & & & $\checkmark$ & & \\
\hline $\mathbf{N A}_{\mathbf{u}}$ & & & & $\checkmark$ & & \\
\hline \multicolumn{7}{|c|}{ Replacement within the same component } \\
\hline DE & $\checkmark$ & & & $\checkmark$ & & \\
\hline $\mathbf{R P}$ & & & & $\checkmark$ & & \\
\hline \multicolumn{7}{|c|}{ Passive modification } \\
\hline BS & $\checkmark$ & & & $\checkmark$ & & \\
\hline BG & $\checkmark$ & $\checkmark$ & $\checkmark$ & & & \\
\hline $\mathbf{B G}_{\mathbf{u}}$ & $\checkmark$ & $\checkmark$ & $\checkmark$ & $\checkmark$ & $\checkmark$ & $\checkmark$ \\
\hline
\end{tabular}

Table 9.1: Payoff properties satisfying the variants.

We used property DA (disjoint additivity) for both frameworks. It states that the sum of payoffs of agent $i$ with respect to two $i$-disjoint networks, is equal to $i$ 's payoff with respect to the union of both networks. In other words, this property excludes the presence of externalities. It can easily be verified that DA is satisfied by all specific models. Observe that DA can be split into the following two properties. 
Property DPA We say that a payoff function $\pi_{i}$ is disjoint superadditive (DPA for short), if for each two networks $g$ and $g^{\prime}$, disjoint w.r.t. agent $i$, we have

$$
\pi_{i}\left(g+g^{\prime}\right) \geq \pi_{i}(g)+\pi_{i}\left(g^{\prime}\right) .
$$

Property DBA We say that a payoff function $\pi_{i}$ is disjoint subadditive (DBA for short), if for each two networks $g$ and $g^{\prime}$, disjoint w.r.t. agent $i$, we have

$$
\pi_{i}\left(g+g^{\prime}\right) \leq \pi_{i}(g)+\pi_{i}\left(g^{\prime}\right) .
$$

We could have used DPA and DBA instead of DA for proving Theorems 4.3.1 and 7.2.3. However, we were unable to weaken DA to either disjoint super- or subadditivity. It would be interesting to examine whether the existence of Nash networks can be proved in another way by using either DPA or DBA.

Properties NA and $\mathbf{N A}_{\mathbf{u}}$ are 'natural' for the one- respectively two-way flow model: linking up with an agent who is already observed is not profitable (at least, when link costs are positive). However, for models with decay this may be profitable, because by an extra link, the distance of several observation paths may be shorter (less decay). For models with imperfectly reliable links, an extra link may also be profitable, because it creates additional observation paths.

We saw that property DE (downstream efficiency) is intuitive for the one-way flow model without decay. By downstreaming a link, an agent still observes all agents whom he already observed, and moreover, he may observe new agents. In the two-way flow model, DE is trivially satisfied (at least, with owner-homogeneous link costs) because by downstreaming a link, an agent observes exactly the same set of agents, i.e. all agents who are active in that component. As an alternative, we proposed property $\mathbf{R P}$, which is demanding but necessary for our line of proof.

Designing properties that are based on replacements for the B\&G-1d and the B\&G-1r model is difficult, because of two opposite effects: on the one hand, downstreaming a link could be profitable by the argument we just mentioned; on the other hand, by upstreaming a link, the directed path from at least one agent will be shortened, which could be profitable in both the B\&G-1d model (less decay), and the B\&G-1r model (higher reliability).

For the B\&G-2d and the B\&G-2r model, a replacement of $(k, i)$ by $(j, i)$ within the same component is profitable (or beneficial) if $j$ is located centrally in that component. By this central location, $i$ is connected to the other agents in that component via short undirected paths, which is profitable in both the model with decay and with imperfectly reliable links. For this reason, Nash networks are often characterized in the literature as stars in the B\&G-2d model. However, in the B\&G-2r model, stars are usually not supported as Nash networks, because agents rather prefer higher connected networks.

As for the properties that concern passive modifications $\left(\mathbf{B S}, \mathbf{B G}\right.$, and $\left.\mathbf{B} \mathbf{G}_{\mathbf{u}}\right)$, observe that in models with decay and/or imperfectly reliable links, higher connected 
networks are preferred over lower connected networks. Extra links that we called 'redundant' (for instance spokes) will be of value since they may create additional observation paths (higher reliability) which may be shorter (less decay).

\subsection{Further research}

Let us conclude with several recommendations for further research.

In the literature on models of unilateral network formation, the concept of Nash networks is a standard equilibrium concept. In Chapter 4, we proposed the concept of local-Nash networks. Observe that the definition of equilibrium networks directly follows from the action spaces that the agents have. It would be interesting to examine other action spaces and the corresponding equilibrium networks.

Recently, several models are proposed in the literature where agents not only choose which links they want to form, but also how much they invest in each link. It would be interesting to study these models more extensively. In Chapter 8, we proposed a model that is inspired by one of these models. In Section 8.5 we discussed how this model is related to similar models, and what further research could be done.

Models of network formation can be enhanced by modeling the behavior of the agents in more realistic ways. In the models that we studied in this thesis, agents are assumed to be rational and myopic. In reality, persons or firms do not always behave completely rational, and it is possible that they think a few of steps ahead. It would be interesting to take these aspects into account.

In real social and economic networks, persons or firms do not always have complete information. They may not know how much valuable information the others have, and moreover, they may not even know who the others in the network are. In view of applications, network formation under incomplete information is a real challenge to be addressed in further studies. 


\section{Bibliography}

Aumann, R., Myerson, R., 1988. Endogenous formation of links between players and coalitions. In: Roth, A. (Ed.), The Shapley Value. Cambridge University Press.

Bala, V., Goyal, S., 2000a. A non-cooperative model of network formation. Econometrica $68,1181-1229$.

Bala, V., Goyal, S., 2000b. A strategic analysis of network reliability. Review of Economic Design 5, 205-228.

Baron, R., Durieu, J., Haller, H., Savani, R., Solal, P., April 2008. Good neighbors are hard to find: computational complexity of network formation. Review of Economic Design 12 (1), 1-19.

Belleflamme, P., Bloch, F., 2004. Market sharing agreements and collusive networks. International Economic Review 45 (2), 387-411.

Bergantiños, G., Lorenzo, L., 2004. A non-cooperative approach to the cost spanning tree problem. Mathematica Methods of Operations Research 59, 393-403.

Billand, P., Bravard, C., Sarangi, S., 2008. Existence of Nash networks in one-way flow models. Economic Theory 37 (3), 491-507.

Bloch, F., Dutta, B., 2009. Communication networks with endogenous link strength, Games and Economic Behavior 66 (1), 39-56.

Bloch, F., Jackson, M. O., 2007. The formation of networks with transfers among players. Journal of Economic Theory 133, 83-110.

Derks, J., Kuipers, J., Tennekes, M., 2009a. An axiomatic approach covering the twoway flow model of network formation. Tech. rep., Maastricht University, Department of Knowledge Engineering.

Derks, J., Kuipers, J., Tennekes, M., Thuijsman, F., 2008a. Existence of Nash networks in the one-way flow model of network formation. In: Neogy S. K. et al. (eds.), Modeling, Computation and Optimization, World Scientific, Ch. 2. 
Derks, J., Kuipers, J., Tennekes, M., Thuijsman, F., 2008b. Local dynamics in network formation. Tech. rep., Maastricht University, Department of Knowledge Engineering.

Derks, J., Kuipers, J., Tennekes, M., Thuijsman, F., 2009b. Project stability. Tech. rep., Maastricht University, Department of Knowledge Engineering.

Derks, J., Tennekes, M., 2007. Payoff functions in the one-way flow model of network formation for which Nash networks exist. Tech. rep., Maastricht University, Department of Knowledge Engineering.

Derks, J., Tennekes, M., 2008. A note on the existence of Nash networks in one-way flow models. Economic Theory 41 (3), 515-522.

Dutta, B., Muttuswami, S., 1997. Stable networks. Journal of Economic Theory 76, 322-344.

Erdős, P., Rényi, A., 1960. On the evolution of random graphs. Publ. Math. Inst. Hung. Acad. Sci 5, 17-61.

Fabrikant, A., Luthra, A., Maneva, E., Papadimitriou, C. H., Shenker, S., 2003. On a network creation game. In: PODC '03: Proceedings of the twenty-second annual symposium on Principles of distributed computing. ACM Press, 347-351.

Falk, A., Kosfeld, M., 2003. It's all about connections: Evidence on network formation. IZA Discussion Papers 777, University of Zurich, IZA.

Feri, F., 2007. Stochastic stability in networks with decay. Journal of Economic Theory 135 (1), 442-457.

Furusawa, T., Konishi, H., 2005. Free trade networks with transfers. Japanese Economic Review 56, 144-164.

Furusawa, T., Konishi, H., 2007. Free trade networks. Journal of International Economics $72(2), 310-335$.

Galeotti, A., 2006. One-way flow networks: the role of heterogeneity. Economic Theory 29 (1), 163-179.

Galeotti, A., Goyal, S., Kamphorst, J., 2006. Network formation with heterogeneous players. Games and Economic Behavior 54 (2), 353-372.

Garey, M., Johnson, D., 1979. Computers and Intractability: A Guide to the Theory of NP-Completeness. Freeman, San Francisco.

Goeree, J. K., Riedl, A., Ule, A., 2009. In search of stars: Network formation among heterogeneous agents. Games and Economic Behavior 67 (2), 445-466. 
Goyal, S., Joshi, S., 2003. Networks of collaboration in oligopoly. Games and Economic Behavior 43, 57-85.

Goyal, S., Joshi, S., 2006. Bilateralism and free trade. International Economic Review 47 (3), 749-778.

Goyal, S., Moraga-Gonzalez, J. L., 2001. R\&D networks. RAND Journal of Economics $32(4), 686-707$.

Grötschel, M., Lovász, L., Schrijver, A., 1981. The ellipsoid method and its consequences in combinatorial optimization. Combinatorica 1 (2), 169-197, corrigendum: Combinatorica 4(4): 291-295 (1984).

Haller, H., Kamphorst, J., Sarangi, S., 2007. (Non-)existence and scope of Nash networks. Economic Theory 31 (3), 597-604.

Haller, H., Sarangi, S., 2005. Nash networks with heterogeneous links. Mathematical Social Sciences 50 (2), 181-201.

Iwata, S., Fleischer, L., Fujishige, S., 2001. A combinatorial strongly polynomial algorithm for minimizing submodular functions. Journal of the ACM 48 (4), 761-777.

Jackson, M. O., 2005. A survey of the formation of networks: stability and efficiency. In: Demange, G., Wooders, M. (Eds.), Group Formation in Economics; Networks, Clubs and Coalitions. Cambridge University Press, Ch. 1.

Jackson, M. O., Van den Nouweland, A., 2005. Strongly stable networks. Games and Economic Behavior 51 (2), 420-444.

Jackson, M. O., Watts, A., 2002. The evolution of social and economic networks. Journal of Economic Theory 106 (2), 265-295.

Jackson, M. O., Wolinsky, A., 1996. A strategic model of social and economic networks. Journal of Economic Theory 71 (1), 44-74.

Kamphorst, J., Van der Laan, G., 2007. Network formation under heterogeneous costs: the multiple group model. International Game Theory Review 9 (4), 599-635.

Kannan, R., Ray, L., Sarangi, S., 2007. The structure of information networks. Economic Theory 30 (1), 119-134.

Karp, R. M., 1972. Reducibility Among Combinatorial Problems. In: Miller, R. E. and Thatcher, J. W. (Eds.), Complexity of Computer Computations. Plenum Press, New York, 85-103.

Kosfeld, M., 2004. Economic networks in the laboratory: A survey. Review of Network Economics 3 (1), 20-42. 
Koster, M., Reijnierse, H., Voorneveld, M., 2003. Voluntary contributions to multiple public projects. Journal of Public Economic Theory 5 (1), 25-50.

Lee, H., Nemhauser, G., Wang, Y., 1996. Maximizing a submodular function by integer programming: Polyhedral results for the quadratic case. European Journal of Operational Research 94, 154-166.

Lovasz, L., 1983. Submodular functions and convexity. In: Bachem, A., Grötschel, M., Korte, B. (Eds.), Mathematical Programming: The State of the Art. Springer, Berlin, 235-257.

Myerson, R., 1977. Graphs and cooperation in games. Mathematics of Operations Research 2, 225-229.

Myerson, R., 1991. Game Theory: Analysis of Conflict. Harvard University Press, Cambridge, Massachusetts.

Nash, J., 1950. Equilibrium points in $n$-person games. Proceedings of the National Academy of Sciences 36, 48-49.

Nemhauser, G., Wolsey, L., Fisher, M., 1978. An analysis of approximations for maximizing submodular set functions - I. Mathematical Programming 14, 265-294.

Neumann, J. von, Morgenstern, O., 1944. Theory of Games and Economic Behavior. Princeton University Press, New Jersey.

Nouweland, A. van den, 2005. Models of network formation in cooperative games. In: Demange, G., Wooders, M. (Eds.), Group Formation in Economics; Networks, Clubs and Coalitions. Cambridge University Press, Ch. 2.

Page, F., Kamat, S., 2005. Farsighted stability in network formation. In: Demange, G., Wooders, M. (Eds.), Group Formation in Economics; Networks, Clubs and Coalitions. Cambridge University Press, Ch. 3.

Rogers, B., 2006. A strategic theory of network status, mimeo. MEDS, Northwestern University.

Schrijver, A., 2000. A combinatorial algorithm minimizing submodular functions in strongly polynomial time. Journal of Combinatorial Theory Series B 80 (2), 346355 .

Schram, A., Offerman, T., Sonnemans, J., 2008. Explaining the Comparative Statics in Step-Level Public Good Games. Vol. 1 of Handbook of Experimental Economics Results. Elsevier, Ch. 86, 817-824.

Tarjan, R., 1972. Depth-first search and linear graph algorithms. SIAM Journal of Computing 1 (2), 146-160. 
Tijs, S., Brânzei, R., 2004. Cost sharing in a joint project. In: Carraro, C., Fragnelli, V. (Eds.), Game Theory and the Environment, Proceedings of the Conference held in Alessandria. Edward Elgar, Cheltenham (UK), 113-124.

Watts, A., February 2001. A dynamic model of network formation. Games and Economic Behavior 34 (2), 331-341.

Zirulia, L., 2006. Industry profit maximizing R and D networks. Economics Bulletin 12 (1), 1-6. 



\section{Notations and definitions}

In Table 9.2, we provide an overview of notations that are used throughout this thesis (except chapter 8). A list of definitions is provided in Table 9.3. For other definitions, we refer to the Index on page 127.

\begin{tabular}{ll}
\hline Basics & \\
$N=\{1,2, \ldots, n\}$ & set of agents \\
$(j, i)$ & link owned by $i$, depicted as $j \bullet \longrightarrow \bullet i$ or $j \bullet-+\bullet$ \\
$g \subseteq N \times N$ where $(i, i) \notin g, \forall i \in N$ & network, defined as a set of links \\
\hline
\end{tabular}

Network information

$N_{i}(g)=\{j \in N:$ a directed path from $j$ to $i$ exists in $g\}$

$N_{i}^{u}(g)=\{j \in N:$ an undirected path between $j$ and $i$ exists in $g\}$

$N_{i}^{d}(g)=\{j \in N:(j, i) \in g\}$

$\operatorname{Car}(g)$, the carrier of $g \quad$ the set of active agents in $g$, being those agents who are begin- or endpoints of a link in $g$

\begin{tabular}{|c|c|}
\hline Network operations & \\
\hline $\begin{array}{l}g+g^{\prime} \\
g+(j, i) \\
g-g^{\prime} \\
g-(j, i) \\
g_{-i} \\
g^{j}=\{(k, \ell):(k, \ell) \in g, \text { and an undirec } \\
g_{-i}^{j}=\left(g_{-i}\right)^{j} \\
g_{-i j}=g_{-i}^{j}+(j, i)\end{array}$ & $\begin{array}{l}\text { notation for } g \cup g \\
\text { notation for } g \cup\{(j, i)\} \\
\text { notation for } g \backslash g^{\prime} \\
\text { notation for } g \backslash\{(j, i)\} \\
\text { network } g \text { minus all links owned by agent } i \\
\text { ed path between } \ell \text { and } j \text { exists in } g\} \\
\text { component of } g \text { where } j \text { is active } \\
\text { component of } g_{-i} \text { where } j \text { is active }\end{array}$ \\
\hline $\begin{array}{l}\quad \text { Payoffs } \\
\pi_{i}: \mathcal{G} \rightarrow \mathbb{R} \text { where } \mathcal{G} \text { is the set of all } \\
\text { networks on } N \\
\pi=\left(\pi_{i}\right)_{i \in N} \\
c_{i j} \\
c_{i j}=c_{i} \text { for all } i, j \in N \\
c_{i j}=c \text { for all } i, j \in N \\
v_{i j} \\
\pi_{i}(g)=\sum_{j \in N_{i}(g)} v_{i j}-\sum_{j \in N_{i}^{d}(g)} c_{i j} \\
\pi_{i}(g)=\sum_{j \in N_{i}^{u}(g)} v_{i j}-\sum_{j \in N_{i}^{d}(g)} c_{i j}\end{array}$ & $\begin{array}{l}\text { payoff function for agent } i \\
\text { joint payoff function } \\
\text { cost of link }(j, i) \\
\text { owner-homogeneous link costs } \\
\text { homogeneous link costs } \\
\text { profit that } i \text { receives from } j \\
\text { B\&G-1 function for agent } i \\
\text { B\&G-2 function for agent } i\end{array}$ \\
\hline
\end{tabular}

Table 9.2: Notations 
Strategy

- global case -

(global) action

best (global) response

(Global) action of agent $i$

$S^{*}$ is a best (global) response of agent $i$ w.r.t. $\pi_{i}$ and $g$

(global-)Nash network Network $g$ is a (global-)Nash network

strict (global-)Nash

network

Network $g$ is a strict

(global-)Nash network

- local case -

local action

Local action of agent $i$ in network $g$

best local response

good local response $S^{*}$ is a best local response of agent $i$ w.r.t. $\pi_{i}$ and $g$

$S^{*}$ is a good local response of agent $i$ w.r.t. $\pi_{i}$ and $g$

neutral local response $S^{*}$ is a neutral local response of agent $i$ w.r.t. $\pi_{i}$ and $g$

local-Nash network

strict local-Nash

network

Network $g$ is a local-Nash network

Network $g$ is a strict local-Nash network
$S \subseteq N \backslash\{i\}$

if $\pi_{i}\left(g_{-i}+\left\{(j, i): j \in S^{*}\right\}\right) \geq \pi_{i}\left(g_{-i}+\right.$ $\{(j, i): j \in S\}$ ) for all (global) actions $S$.

if $N_{i}^{d}(g)$ is a best (global) response in $g$ for each $i \in N$.

if $N_{i}^{d}(g)$ is the unique best (global) response in $g$ for each $i \in N$.

$S \subseteq N \backslash\{i\}$ where $\left|N_{i}^{d}(g) \backslash S\right| \leq 1$ and $\left|S \backslash N_{i}^{d}(g)\right| \leq 1$.

if $\pi_{i}\left(g_{-i}+\left\{(j, i): j \in S^{*}\right\}\right) \geq \pi_{i}\left(g_{-i}+\right.$ $\{(j, i): j \in S\})$ for all local actions $S$.

if $\pi_{i}\left(g_{-i}+\left\{(j, i): j \in S^{*}\right\}\right) \geq \pi_{i}(g)$.

if $\pi_{i}\left(g_{-i}+\left\{(j, i): j \in S^{*}\right\}\right)=\pi_{i}(g)$.

if $N_{i}^{d}(g)$ is a best local response in $g$ for each $i \in N$.

if $N_{i}^{d}(g)$ is the unique best local response in $g$ for each $i \in N$.

Link/network evaluations

profitability

beneficiality

\section{Network architectural properties}

minimal

minimal

(undirected case)

proper

semi-proper

cycle-proper
Link $(j, i) \in g$ is profitable in $g$

Link $(j, i) \notin g$ is profitable in $g$

Link $(j, i)$ is beneficial in $g$

Network $g$ is beneficial if $\pi_{i}(g) \geq \pi_{i}(g-(j, i))$.

if $\pi_{i}(g+(j, i)) \geq \pi_{i}(g)$.

if $\pi_{i}\left(g_{-i j}\right) \geq \pi_{i}\left(g_{-i}^{j}\right)$.

if all links in $g$ are beneficial.
Network $g$ is minimal

Network $g$ is minimal

(undirected case)

Network $g$ is proper

Network $g$ is semi-proper

Network $g$ is cycle-proper if for each $(j, i) \in g$ an additional directed path from $j$ to $i$ does not exist in $g$.

if for each $(j, i) \in g$ an additional undirected path between $j$ and $i$ does not exist in $g$.

if each agent has at most one outgoing link. if for all $(i, j),(i, k) \in g$, with $j \neq k$, link $(i, j)$ is contained in a cycle, and link $(i, k)$ is contained in a cycle.

if each agent on a cycle has exactly one outgoing link. 


\section{Index}

action, 12

best local response, 53,72

best response, 12

good local response, 53,72

local, 53

neutral local response, 72

agent, 3, 11

active, 11,52

leaf, 27

observed, 5,11

observed (undirected case), 93

representative, 41

root-, 42

top-, 54

algorithm

BRUTE-FORCE, 40

ARRAC, 42

$\mathrm{RCR}, 50$

space complexity, 44

time complexity, 44

beneficiality, 16, 55,94

best local response, 53,72

best response, 12

Best Response Problem, 36,50

big $\mathrm{O}$ notation, 44

bilateral connections model, 4

connected set, 41

connection number, 59

connections model, 4

cooperative game, 3,109

cycle, 11

cycle network, 13 cycle-proper network, 33

decay, see information decay

decision problem, 36

directed path, 11

disjoint networks, 55

dynamic procedure, 75

endogenous network, 2

equilibrium network, 3

exogenous network, 2

feasible project, 106

game, 3

global action, see action

global-Nash network, see Nash network

good local response, 53,72

grand project, 106

group, 41

heterogeneity, 12

homogeneity, 12

information decay, 5,115

investment profile, 106

joint payoff function, 11

link, 1

beneficial, 55,94

cost, 12, 93

own, 5,12

profitable, 55, 94

spoke, 59, 104

local action, 53 
local-Nash network, 53

minimal network, 24,62

modularity, 38

Nash equilibrium, 5, 112

Nash network, 5,12

network, 1, 11

beneficial, 55,95

component, 11, 52

cycle, 13

cycle-proper, 33

local-Nash, 53

minimal, 24,62

minimal (undirected case), 93

Nash, 5, 12

proper, 17, 28, 54

semi-proper, 25

strict local-Nash, 62

strict Nash, 5, 12, 27

strongly connected, 24

network formation game, 3

neutral local response, 72

non-cooperative game, 3,12

nonrival profits, 5

NP class, 37

NP-hard class, 37

observed agent, 5, 11

observed agent (undirected case), 93

one-way flow game, 13

one-way flow model, 5,9

optimization problem, 36

orderly payoff function, 59

outdegree, 11

owner-homogeneity, 12

$\mathrm{P}$ class, 37

pairwise stability, 4

payoff, 112

payoff function, 5, 11,53

B\&G-1, 12, 36, 82

B\&G-2, 50,93
BF (beneficial farthest), 58

BG (beneficial growth), 59,97

BG2 (beneficial growth, undir.), 98

BS (beneficial shrink), 59

BT (beneficial topagent), 58

DA (disjoint additivity), 55, 94

DBA (disjoint subadditivity), 117

DE (downstream efficiency), 42, 56

DPA (disjoint super additivity), 117 joint, 11

NA (naturality), 56, 95

NA2 (naturality, undirected case), 95

orderly, 59

$\mathrm{RP}$ (replacement), 98

payoff set function, 38

personal project, 114

procedure, 75

profit, 12,93

profitability, 55, 94

project, 106

project support game, 106

proper network, 17, 28, 54

reliability of links, 5,115

semi-proper network, 25

space complexity, 44

spoke, 59,104

stable investments, 107

strict local-Nash network, 53

strict Nash network, 5, 12, 27

strongly connected network, 24

submodularity, 38

supermodularity, 38

task, 106

termination, 73,75

time complexity, 44

topagent, 54

two-way flow model, 5, 49,91

undirected path, 11

unilateral connections model, 4, 115 


\section{Samenvatting}

Dit proefschrift behandelt wiskundige modellen van netwerkformatie. Hierbij gaat het om zogenaamde endogene netwerken, dat wil zeggen, netwerken die worden gevormd door individuen die vertegenwoordigd worden door knopen. Een voorbeeld hiervan is een vriendschapsnetwerk, waarin een verbinding correspondeert met de vriendschap tussen twee personen. De personen formeren zelf een vriendschapsnetwerk door onderlinge vriendschappen te sluiten. Een ander voorbeeld is een handelsnetwerk, waarin een verbinding een handelsrelatie vertegenwoordigt tussen twee handelaren.

We zijn geïnteresseerd in het formatieproces van endogene netwerken. Om dit te onderzoeken, gebruiken we vereenvoudigde modellen uit de speltheorie. Deze modellen worden netwerkformatiespelen (network formation games) genoemd. De agenten, die corresponderen met de knopen, leggen verbindingen volgens een bepaalde procedure. Aan het einde van deze procedure ontvangt elke agent een bepaalde uitbetaling aan de hand van het uiteindelijk gevormde netwerk. Centrale onderzoeksvragen die worden gesteld in dit proefschrift zijn: onder welke voorwaarden bestaan evenwichtsnetwerken (d.w.z. netwerken waarbij geen één agent zich kan verbeteren) en indien ze bestaan, welke topologie hebben ze? Verder onderzoeken we welke procedures tot evenwichtsnetwerken leiden.

Als basismodel in Hoofdstukken 2 tot en met 7 gebruiken we het unilaterale verbindingsmodel (unilateral connections model), dat is ontwikkeld door Bala en Goyal (2000a). De agenten formeren een netwerk door simultaan een verzameling gerichte verbindingen te kiezen, waarbij elke agent alleen kan kiezen uit de verbindingen die naar hem gericht zijn. Alle gekozen verbindingen vormen samen het uiteindelijke netwerk. Op basis van dit netwerk ontvangt elke agent een bepaalde uitbetaling. Een verzameling verbindingen die een agent kiest, wordt een actie genoemd. Een actie van een bepaalde agent wordt een beste antwoord genoemd wanneer hij, gegeven de acties van alle andere agenten, de hoogst mogelijke uitbetaling ontvangt. Een Nash netwerk is een netwerk waarin iedere speler een beste antwoord speelt, en een strikt Nash netwerk is een netwerk waarin iedere speler een uniek beste antwoord speelt.

In Hoofdstuk 2 bestuderen we het eenrichtingsstroommodel (one-way flow model), dat wordt gekenmerkt door de volgende uitbetalingsfunctie: iedere agent betaalt bepaalde kosten voor elke verbinding die hij heeft gelegd en ontvangt bepaalde opbreng- 
sten van andere agenten. Deze opbrengsten zijn niet rivaliserend, dat wil zeggen dat wanneer een agent ze doorgeeft aan anderen, dan heeft hij er nog steeds beschikking over. Als voorbeeld hiervan kan gedacht worden aan informatie. In het eenrichtingsstroommodel ontvangt agent $i$ opbrengsten van agent $j$ als er een gericht pad is van $j$ naar $i$; de opbrengsten stromen als het ware naar $i$ toe.

Hoofdstuk 2 richt zich op het bestaan en de topologie van Nash netwerken. We leveren twee verschillende bewijzen voor het bestaan van Nash netwerken waarbij verbindingen, die tot dezelfde eigenaar behoren, gelijke kosten hebben. Met een tegenvoorbeeld laten we zien dat Nash netwerken niet altijd bestaan voor spelen met heterogene verbindingskosten. We laten zien dat Nash netwerken wel bestaan onder bepaalde condities. Tevens karakteriseren we de topologie van Nash netwerken en strikt Nash netwerken voor homogene en heterogene verbindingskosten. Ook beschouwen we spelen waarbij opbrengsten negatief kunnen zijn. Dit houdt in dat er bijvoorbeeld ongewenste informatie door een netwerk kan circuleren. Voor deze spelen laten we zien dat efficiënte netwerken (waarin de totale uitbetaling maximaal is) niet altijd Nash netwerken zijn. Bovendien laten we zien dat Nash netwerken niet altijd bestaan, zelfs wanneer de verbindingskosten homogeen zijn. We besluiten Hoofdstuk 2 met een overzicht van de resultaten die betrekking hebben op het eenrichtingsstroommodel.

Het vinden van beste antwoorden in het eenrichtingsstroommodel wordt bestudeerd in Hoofdstuk 3. We bewijzen dat dit probleem, genaamd het beste-antwoord-probleem (best response problem), NP-moeilijk is, wat min of meer wil zeggen dat een oplossing niet efficiënt te berekenen is. Hierbij gebruiken we een methode waarbij elke instantie van het bekende NP-moeilijke probleem minimale verzamelingsdekking (minimum set cover) vertaald kan worden naar een instantie van het beste-antwoord-probleem.

We introduceren een algoritme, genaamd ARRAC, om een beste antwoord te vinden en vergelijken dit met BRUTE-FORCE (brute kracht), een algoritme dat voor iedere mogelijke actie de uitbetaling berekent om zodoende een beste antwoord eruit te kiezen. Algoritme ARRAC is gebaseerd op drie technieken om het mogelijk aantal acties te reduceren. De tijdscomplexiteit van ARRAC is identiek aan die van BRUTE-FORCE. Echter, door middel van experimenten laten we zien dat ARRAC wel degelijk efficiënter is. Tenslotte bestuderen we het beste antwoord probleem met betrekking tot het tweerichtingsstroommodel (two-way flow model). In dit model ontvangt agent $i$ opbrengsten van agent $j$ als er een ongericht pad is tussen $i$ en $j$. Aan de hand van een algoritme laten we zien dat dit probleem in polynomiale tijd oplosbaar is.

In Hoofdstuk 4 wordt het unilaterale verbindingsmodel aangepast door de acties van de agenten te beperken tot lokale acties, welke zijn: het toevoegen, verplaatsen of verwijderen van één verbinding, of niets doen. Een lokaal-Nash netwerk wordt gedefinieerd als een netwerk waarin niemand een lokale verbeteractie kan uitvoeren. Een netwerk dat in Hoofdstuk 2 een Nash netwerk werd genoemd, wordt nu een globaalNash netwerk genoemd.

We introduceren axiomatische uitbetalingseigenschappen die geïnspireerd zijn op het eenrichtingsstroommodel. Voor uitbetalingsfuncties die aan enkele van deze ei- 
genschappen voldoen, bewijzen we dat lokaal-Nash netwerken die proper zijn, d.w.z. waarin iedere agent hoogstens één uitgaande verbinding heeft, ook globaal-Nash zijn. Daarna bewijzen we de existentie van lokaal-Nash netwerken voor uitbetalingsfuncties die aan het raamwerk van eigenschappen voldoen. Met een voorbeeld tonen we aan dat ook niet-propere netwerken lokaal-Nash kunnen zijn. We bewijzen dat de uitbetalingseigenschappen onafhankelijk van elkaar zijn. Tenslotte relateren we het raamwerk van uitbetalingseigenschappen aan het eenrichtingsstroommodel en geven we een aantal voorbeelden van uitbetalingsfuncties die niet tot het eenrichtingsstroommodel behoren, maar die wel voldoen aan alle eigenschappen.

Een dynamisch spel, waarin agenten om beurten lokale acties spelen, wordt bestudeerd in Hoofdstuk 5. Het spel begint met een willekeurig netwerk. Dan wordt er willekeurig een agent geselecteerd, die een lokale actie mag spelen. Dit wordt herhaald totdat een netwerk wordt bereikt dat niemand wil veranderen. Iedere agent krijgt een uitbetaling op basis van dit uiteindelijke netwerk. De vraag die wordt gesteld in dit hoofdstuk, is of het spel altijd eindigt, met andere woorden, of er een netwerk wordt bereikt waarin niemand een lokale verbeteractie kan spelen. Om deze vraag te beantwoorden, bestuderen we een procedure van willekeurig geselecteerde lokale acties. We bewijzen dat deze procedure altijd eindigt als aan alle uitbetalingseigenschappen uit Hoofdstuk 4 wordt voldaan. Het netwerk dat uiteindelijk wordt bereikt is lokaal-Nash en tevens globaal-Nash.

In Hoofdstuk 6 worden de uitbetalingseigenschappen uit Hoofdstuk 4 gerelateerd aan het eenrichtingsstroommodel. We geven een volledige karakterisering van de uitbetalingsfuncties van het eenrichtingsstroommodel die voldoen aan alle eigenschappen uit Hoofdstuk 4. Hierbij beschouwen we uitbetalingsfuncties waarbij verbindingskosten en opbrengsten negatief mogen zijn. We laten zien dat een deelklasse van functies met heterogene verbindingskosten en opbrengsten aan de eigenschappen voldoet waarvoor we het bestaan van lokaal-Nash netwerken hebben bewezen. Verder laten we zien dat elke functie met homogene niet-negatieve verbindingskosten en heterogene niet-negatieve opbrengsten voldoet aan alle eigenschappen.

Het tweerichtingsstroommodel (two-way flow model) wordt bestudeerd in Hoofdstuk 7 . Het enige verschil met het eenrichtingsstroommodel is dat agent $i$ opbrengsten ontvangt van agent $j$ als er een ongericht pad is tussen $i$ en $j$. In Hoofdstuk 7 gebruiken we dezelfde aanpak als in Hoofdstuk 4. Eerst bewijzen we dat voor uitbetalingsfuncties die aan bepaalde axiomatische eigenschappen voldoen, minimale lokaal-Nash netwerken ook globaal-Nash zijn. Met een minimaal netwerk bedoelen we dat de ongerichte versie ervan geen enkele cykel bevat. Daarna bewijzen we de existentie van minimale lokaal-Nash netwerken voor uitbetalingseigenschappen die aan een raamwerk van eigenschappen voldoen. We laten zien dat uitbetalingsfuncties waarbij de verbindingen, die tot dezelfde eigenaar behoren, gelijke kosten hebben en waarbij de opbrengsten negatief kunnen zijn, aan alle eigenschappen voldoen. Tevens geven we een aantal voorbeelden van uitbetalingsfuncties die niet tot het tweerichtingsstroommodel behoren, maar die wel voldoen aan alle eigenschappen. 
In Hoofdstuk 8 bestuderen we een model waarin de financiering van een groot project door een groep agenten wordt onderzocht. Dit grote project bestaat uit een verzameling taken, die bepaalde kosten met zich meebrengen. Omdat deze kosten aan elkaar gerelateerd zijn, definiëren we een kostenfunctie op de verzameling projecten (hierbij is een project een deelverzameling taken). Tevens levert het uitgevoerde project bepaalde opbrengsten op voor iedere agent. De vraag is of de agenten bereid zijn te investeren in het grote project. Om dit te onderzoeken, laten we elke agent per taak een investering voorstellen. Een project kan alleen worden uitgevoerd als de kosten ervan betaald kunnen worden met de voorgestelde investeringen.

We geven condities waarvoor investeringsprofielen bestaan waarmee het grote project betaald kan worden en waarbij niemand profiteert door af te wijken. Wanneer aan deze condities wordt voldaan, worden de investeringen stabiel genoemd. We laten zien hoe stabiliteit gerelateerd is aan het Nash evenwichtsconcept. Als voorbeeld van ons model beschrijven we een situatie waarin onderzocht wordt of er voldoende animo aanwezig is voor het bouwen van een buurtcentrum.

Alhoewel het model in hoofstuk 8 op het eerste zicht weinig met netwerkformatie te maken lijkt te hebben, is het gebaseerd op een model van netwerkformatie dat is onderzocht door Bloch en Jackson (2007). In dat model formeren de agenten een netwerk door investeringen voor te stellen per verbinding.

In Hoofdstuk 9 besluiten we dit proefschrift met een bespreking van uitbreidingen op het unilaterale verbindingsmodel en doen we aanbevelingen voor verder onderzoek.

In de literatuur is het unilaterale verbindingsmodel op de volgende twee manieren uitgebreid. De eerste uitbreiding is dat opbrengsten die een agent ontvangt van een andere agent in waarde afnemen met de lengte van het verbindingspad tussen hen (gemeten in het aantal verbindingen). De tweede uitbreiding is dat elke verbinding functioneert met een bepaalde betrouwbaarheidskans. We relateren de uitbetalingseigenschappen die we in Hoofdstukken 4 en 7 van dit proefschrift hebben voorgesteld aan deze uitbreidingen.

We eindigen Hoofdstuk 9 met een aantal aanbevelingen voor verder onderzoek. In dit proefschrift en in de meeste relevante literatuur wordt verondersteld dat de agenten volledig rationeel handelen en bovendien wordt verondersteld dat ze volledige kennis hebben van de omgeving. Voor verder onderzoek zou het interessant zijn om deze aannames (deels) te laten vallen. 


\section{Acknowledgements}

From 2004 to 2009, I worked on a Ph.D. project, which resulted in this thesis. This project would not have been possible without the support of many people.

First of all, I would like to express my gratitude to Frank Thuijsman for making me enthusiastic for game theory, and for giving me the opportunity to start this Ph.D. project. Although he would have liked to be more involved in this project, his contribution was valuable. He spread the results of this project all over the world, from Delhi to Chicago.

Second, I want to thank Jeroen Kuipers for his support, especially his precise way of conducting research. Furthermore, I always liked his easy accessibility. The room he shared with Jean always had a great ambiance.

Above all, I want to thank Jean Derks. Especially during the last two years, our cooperation was very successful. My research link with him was more beneficial than any other. This synergy was essential for the formation of this thesis. Due to his 'properties' such as cooperativeness, open-mindedness, and friendliness, it really was a great pleasure for me to work with him.

Also thanks to Ralf Peeters for supporting me at the end of my Ph.D. project.

Further thanks to my other former colleagues of the Department of Knowledge Engineering for the great working atmosphere, especially on Thursdays around 17.30 at the Tribunal.

Furthermore, I want to thank my parents, Peter and Susanne, and Yvonne's parents for their support. I would also like to thank my friends. In particular, Bert for his easygoingness and our snooker evenings and cycling trips, and Remme for his openmindedness and the relaxed and sometimes competitive spirit during our running and cycling trips.

Also thanks to the former coverband 'Not on Thursdays' I played guitar in, for inspiring each other with music, and Tafelstraat 13 for providing a great place to meet people and talk about the meaning of life.

Finally, I want to thank Yvonne for joining me on the path of life, and sharing all things we experience. It is really wonderful to be with somebody who truly understands me and who complements me. 



\section{About the author}

Martijn Tennekes was born in Maarssen, The Netherlands, on the 10th of January, 1981. From 1993 to 1999 he followed secondary education (VWO) at Niftarlake College in Maarssen. Afterwards, he studied knowledge engineering at Maastricht University. In 2004, he graduated with a specialization in operations research. His master's thesis, entitled 'Get Connected!', deals with a game theoretic approach on building networks, where agents manage multiple nodes which they have to connect to a source node. He wrote this thesis under the supervision of Frank Thuijsman and Jeroen Kuipers. In 2004, he was given the opportunity to continue this line of research by a Ph.D. project at the Department of Knowledge Engineering, Maastricht University. The supervision of this project was carried out by Jean Derks, Jeroen Kuipers, and Frank Thuijsman. The results are presented in this thesis. Since April 2009, he is working at the Department of Methodology and Quality at Statistics Netherlands (CBS). 